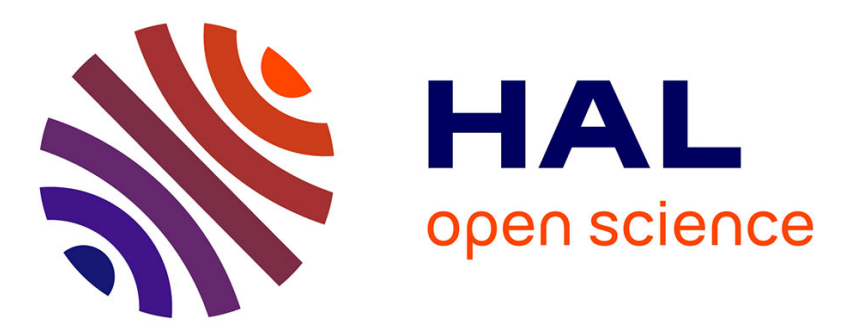

\title{
La plaine de Vientiane au tournant du second millénaire. Données nouvelles sur l'expansion des espaces khmer et môn anciens au Laos (II)
}

Michel Lorrillard

\section{- To cite this version:}

Michel Lorrillard. La plaine de Vientiane au tournant du second millénaire. Données nouvelles sur l'expansion des espaces khmer et môn anciens au Laos (II). Bulletin de l'Ecole française d'ExtrêmeOrient, 2014, 100 (1), pp.38-107. 10.3406/befeo.2014.6167 . halshs-01889969

\section{HAL Id: halshs-01889969 \\ https://shs.hal.science/halshs-01889969}

Submitted on 8 Oct 2018

HAL is a multi-disciplinary open access archive for the deposit and dissemination of scientific research documents, whether they are published or not. The documents may come from teaching and research institutions in France or abroad, or from public or private research centers.
L'archive ouverte pluridisciplinaire HAL, est destinée au dépôt et à la diffusion de documents scientifiques de niveau recherche, publiés ou non, émanant des établissements d'enseignement et de recherche français ou étrangers, des laboratoires publics ou privés. 
La plaine de Vientiane au tournant du second millénaire. Données nouvelles sur l'expansion des espaces khmer et môn anciens au Laos (II)

Michel Lorrillard

\section{Citer ce document / Cite this document :}

Lorrillard Michel. La plaine de Vientiane au tournant du second millénaire. Données nouvelles sur l'expansion des espaces khmer et môn anciens au Laos (II). In: Bulletin de I'Ecole française d'Extrême-Orient. Tome 100, 2014. pp. 38-107; doi : https://doi.org/10.3406/befeo.2014.6167 https://www.persee.fr/doc/befeo_0336-1519_2014_num_100_1_6167

Fichier pdf généré le 08/05/2018 


\title{
Résumé
}

Cette étude sur les espaces khmer et môn de la vallée moyenne du Mékong fait suite à un premier article qui concernait le sud et le centre du Laos, et s'attache maintenant à la plaine de Vientiane. La situation archéologique y est sensiblement différente et montre une influence plus tardive des modèles culturels issus de l'Inde. Seuls des témoignages bouddhiques ont été retrouvés et ceux-ci ne remontent certainement pas au-delà du VIIle siècle. L'occupation mône y est clairement attestée par une trentaine de sites positionnés le long de rivières ou autour de surfaces bien irriguées, principalement la Nam Ngum et le grand marais du That Luang. Pour deux sites rupestres, il semble bien qu'une transition se soit opérée entre la culture mône et la culture angkorienne. Cette dernière a également laissé sur le site de l'actuelle ville de Vientiane des vestiges dont la plupart datent manifestement du règne de Jayavarman VII. Certains de ces vestiges ont pu être apportés, mais les traces d'aménagements anciens montrent qu'une présence khmère a été effective : elle doit alors, tout comme c'est le cas pour l'occupation mône, être liée à des sites de la rive droite du Mékong.

\begin{abstract}
This study devoted to the Khmer and Mon spaces in the middle valley of the Mekong is a follow-up to a previous article concerning Southern and Central Laos, this time focusing on the Vientiane Plain. The situation is significantly different from the archaeological point of view as the influence of the cultural models imported from India appeared at a somewhat later time. Only Buddhist testimonies could be evidenced and they certainly cannot be dated as earlier than the 8th century. The presence of Mon people is clearly evidenced by about 30 sites located alongside rivers or around well irrigated areas, mainly the Nam Ngum and the That Luang Great Swamp. Two rock-shelter sites seem to testify to a shift from Mon to the Angkorian models. Some vestiges of the latter culture have also been identified in the modern city of Vientiane ; most of them can clearly be traced back to the reign of Jayavarman VII. Some of these relics may have been imported into this area, but traces of ancient structures leave no doubt about a Khmer presence which, just like the Mon's, was obviously related to sites on the right bank of the Mekong.
\end{abstract}




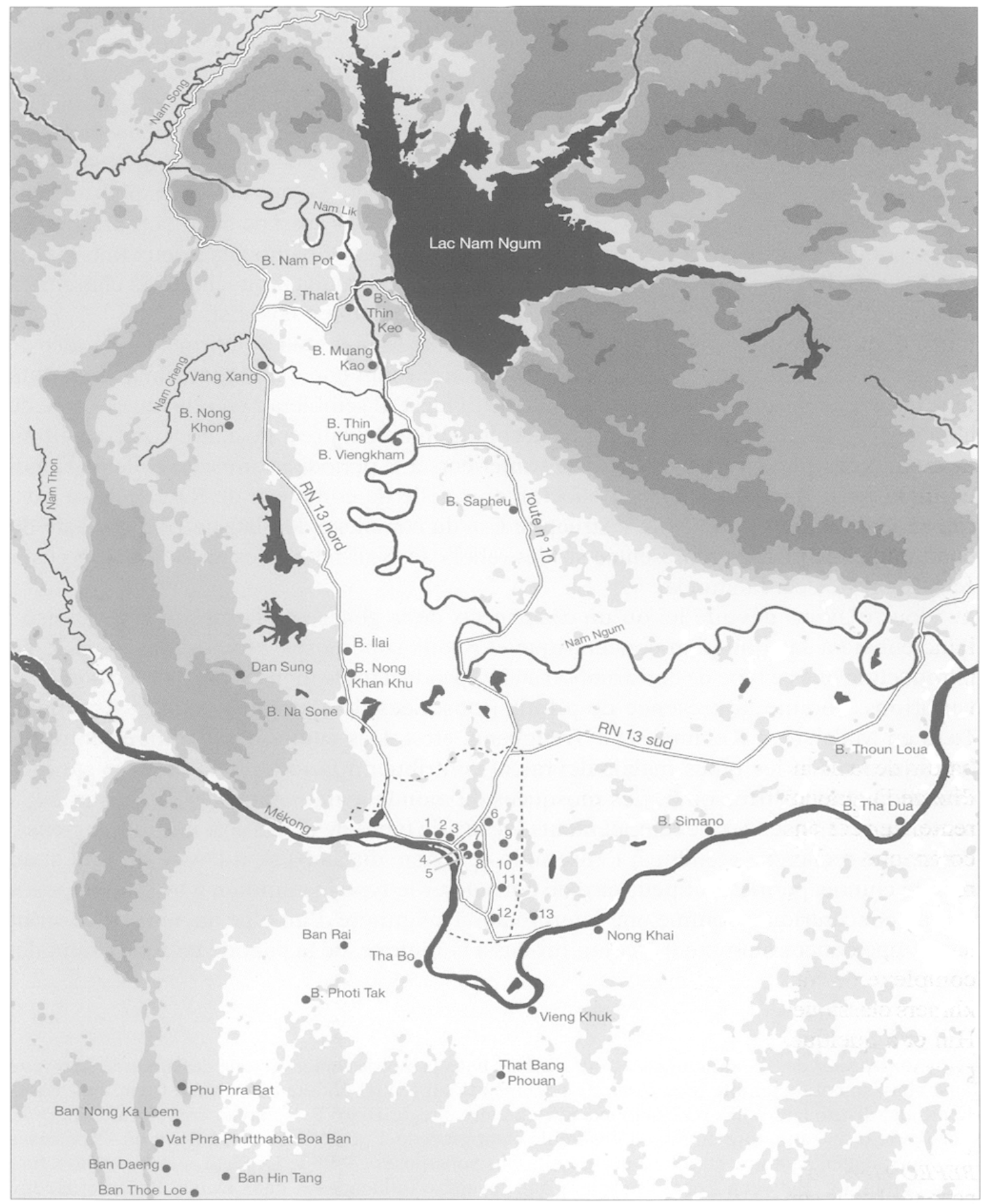

Lieux de découverte de vestiges khmers et môns dans la plaine de Vientiane et ses abords immédiats sur la rive droite du Mékong (M. Lorrillard / J. Ferrand).

1. Vat Inpeng - 2. Vat Mixay - 3. Vat Si Bun Yeun - 4. Vat Kao Yot - 5. Vat Dong Palan - 6. Vat Phon Pa Nao7. That Luang - 8. Ban Saphang Mo - 9. Ban Non Kho - 10. Ban Muang Noy - 11. Ban Don Koy 12. Ban Somsanouk - 13. Ban Dong Phosy 


\title{
La plaine de Vientiane au tournant du second millénaire Données nouvelles sur l'expansion des espaces khmer et môn anciens au Laos (II)
}

\author{
Michel LORRILLARD*
}

Cet article, dont la numérotation de chapitre commence par le chiffre VIII, fait suite à une contribution publiée dans un précédent volume du Bulletin ${ }^{1}$. Nous avions mis en évidence dans celle-ci, au-delà du fameux complexe architectural de Vat Phu sis dans la province de Champassak, la présence dans quatre autres provinces méridionales et centrales du Laos - Attopeu, Saravane, Savannakhet et Khammouane - d'une cinquantaine de sites présentant des vestiges relevant des cultures khmère (préangkorienne et angkorienne) et mône. Nous avons observé que les témoignages préangkoriens se retrouvent de façon quasi continue sur la rive gauche du Mékong depuis la frontière cambodgienne jusqu'au $17^{\mathrm{e}}$ degré de latitude Nord, après quoi ils semblent disparaître ${ }^{2}$. La culture mône, dont les vestiges les plus méridionaux au Laos ont été situés à la limite nord-ouest de la province de Saravane, est quant à elle particulièrement représentée dans la province de Savannakhet. Ce fait n'est en réalité guère étonnant, car cette région de plaine abondamment irriguée (la plus vaste du territoire laotien) n'est que la continuation physique du plateau de Khorat qui occupe tout le nord-est de la Thaïlande. Il a déjà été établi depuis plusieurs décennies que la présence mône - d'abord reconnue dans le bassin de la Ménam Chao Phraya où elle est associée au développement de la civilisation dite de Dvāravatī - s'est également largement répandue, quoique sous une forme différente, sur cet énorme territoire (seize provinces formant une entité géographiquement compacte) de la rive droite du Mékong, en particulier durant la seconde moitié du premier millénaire ${ }^{3}$. Si la province de Champassak bénéficie par ailleurs de la persistance d'une faveur particulière sous le règne des souverains d'Angkor, qui y construisent temples, bassins artificiels, gîtes d'étape et āśrama, nous avons remarqué qu'en amont du complexe de Vat Phu, et cela sur plus de $200 \mathrm{~km}$ du cours du fleuve, les témoignages khmers classiques s'avèrent absents. Il faut remonter jusqu' au sanctuaire inachevé de Huan Hin et à quelques édifices situés à l'intérieur des terres de la province de Savannakhet pour retrouver des vestiges de l'époque angkorienne. Nous avons conclu que le Mékong

* Maître de conférences à l'École française d'Extrême-Orient.

1. «Par-delà Vat Phu. Données nouvelles sur l'expansion des espaces khmer et môn anciens au Laos », $B E F E O 97-98,2010-2011$, p. 205-270. Le lecteur voudra bien excuser l'oubli de cinq cartes, essentielles pour une bonne compréhension du texte, qui devaient accompagner cet article. Elles sont reproduites avant les figures qui illustrent la présente contribution.

2. Cf. également Michel Lorrillard, «Pre-Angkorian Communities in the Middle Mekong Valley (Laos and Adjacent Areas) ", dans Nicolas Revire \& Stephen A. Murphy (éd.), Before Siam: Essays in Art and Archaeology, Bangkok, Siam Society/River Books, 2014, p. 186-215.

3. Pour une présentation actualisée du sujet, cf. la thèse récente de Stephen A. Murphy, The Buddhist Boundary Markers of Northeast Thailand and Central Laos, 7th-12th centuries CE: Towards an Understanding of the Archaeological, Religious and Artistic Landscapes of the Khorat Plateau, Doctoral Dissertation, University of London, School of Oriental and African Studies, 2010. 
n'était certainement plus à cette époque l'axe de communication (nord-sud) privilégié qu'il avait été aux temps préangkoriens. La répartition des sites, tant sur la rive gauche que sur la rive droite, indique au contraire un intérêt plus important pour les routes terrestres, en particulier celles - orientées est-ouest - qui reliaient au travers de passes la côte du centre du Vietnam aux terres les plus continentales de la péninsule Indochinoise. Sur une soixantaine de kilomètres en amont du confluent de la Sé Bang Fai, la province de Khammouane forme sur la rive gauche du fleuve la limite septentrionale d'un espace d'habitat ancien. Les terres basses s'y resserrent considérablement et les reliefs les plus occidentaux de la chaîne Annamitique viennent ici rejoindre le Mékong qu'ils bordent ensuite sur quelque $300 \mathrm{~km}$, interférant par là-même sur son cours. Le stūpa laotien du That Sikhot, à Tha Khaek, recouvre encore probablement un monument angkorien (présence de pièces d'architecture typiques, dont des antéfixes d'angle en forme de nāga), mais il représente alors la dernière étape au nord sur la route du fleuve. Dans la province de Borikhamxai, où le cours du Mékong a été contraint par la configuration géologique d'opérer un énorme coude (à partir de la préfecture de Vientiane, il coule d'abord vers le nord-est, puis vers l'est, enfin vers le sud, obligeant les bateliers qui remontent le fleuve à faire un long et laborieux détour avant de retrouver une plaine sur la rive gauche), aucun vestige antérieur au second millénaire n'a pu être identifié de façon certaine. Cet espace est pourtant le lieu de convergence de plusieurs rivières importantes dont la Nam Hin Boun, la Nam Kading, la Nam San et la Nam Ngiep. Il n'y a guère qu'à l'embouchure de cette dernière que quelques images de Buddha en pierre, toutes amputées de leur partie supérieure, apparaissent comme des témoignages d'un passé relativement ancien. Mais leur facture qui rappelle par certains traits des images de Phayao au Lān Nā indique qu'elles ne peuvent guère être antérieures au $\mathrm{XVI}^{\mathrm{e}}$ siècle et qu'elles relèvent plutôt d'un atelier local et quelque peu en marge. Dans la mesure où la Nam San et la Nam Ngiep prennent leur source sur le plateau de Xieng Khuang, il ne serait d'ailleurs pas étonnant que ces sculptures bouddhiques aient un rapport avec les relais bordant les voies d'eau qui étaient établis entre le royaume septentrional des Tai-Phuan - florissant entre le $\mathrm{XVI}^{\mathrm{e}}$ et le XVIII ${ }^{\mathrm{c}}$ siècle - et celui de Vientiane.

Si la progression vers le nord au départ de la province de Khammouane est rendue difficile par les reliefs qui obstruent la rive gauche du Mékong, il en va différemment si l'on passe sur la rive droite, où s'ouvre le large bassin de la Nam Songkhram et de ses affluents. Une route ancienne orientée plein ouest traverse les provinces thaïlandaises actuelles de Nakhon Phanom, Sakorn Nakhon et Udon Thani, reliant de grands sites entourés de douves et de remparts de forme quadrangulaire dont la tradition mythique a gardé les noms. Il s'agit de Nong Han Luang (actuelle ville de Sakorn Nakhon) et Nong Han Noy (actuel Nong Han), autour desquels existent plusieurs monuments de l'époque angkorienne - en fait les derniers attestés avec certitude pour la limite septentrionale de l'empire khmer. À proximité immédiate de Sakorn Nakhon on rencontre les Prasat Phra That Dum, Phra That Choeng Chum et Phra That Narai Cheng Weng. Plus à l'ouest, sur les hauteurs d'une petite chaîne qui sépare le bassin de la Nam Songkhram de ceux de la Nam Chi et de la Nam Mun, domine le Prasat Phra That Phu Phek. Nong Han se trouve sur le même axe, alors que les sanctuaires d'hôpitaux du Prasat Ban Phan Na et du Prasat $\mathrm{Ku}$ Kaeo Ban Chit sont situés respectivement un peu plus au nord et un peu plus au sud. L'occupation de cette région est en fait très ancienne, comme l'attestent le nombre et la richesse des sites d'habitat préhistoriques - à commencer par celui de Ban Chiang que $15 \mathrm{~km}$ à peine séparent de Nong Han. Ce dernier site, encore parfaitement entouré de sa douve d' $1 \mathrm{~km}$ de côté, semble avoir été l'intersection à partir de laquelle les voyageurs 
de l'est rattrapaient la route qui, venant des bassins méridionaux de la Mun et de la Chi, permettait de rejoindre au nord le Mékong, distant d'une soixantaine de kilomètres, et surtout d'accéder à l'autre grande plaine située sur sa rive gauche, celle de Vientiane ${ }^{4}$.

\section{Les sites de la plaine de Vientiane}

Cette présentation des sites historiques de la plaine de Vientiane antérieurs à la période lao (reconnue pour débuter avec la fondation du royaume du Lān Xāng au milieu du XIV siècle) s'appuie en partie sur les travaux pionniers qui ont été effectués entre 1974 et 1977 par Pierre-Marie Gagneux, en coopération avec le service des Monuments historiques du ministère de l'Éducation, des Sports et des Cultes du Laos. Il sera fréquemment fait référence au rapport ronéotypé dans lequel cet auteur inventorie, en même temps que leur site d'origine, une centaine de vestiges archéologiques supposés être liés à la civilisation mône ${ }^{5}$. Les conditions qui nous permettent d'approcher cette période historique ont cependant changé depuis une quarantaine d'années, et il était important de procéder à une mise à jour complète des données. Un certain nombre des vestiges qui avaient été relevés in situ avant 1977 ne sont plus visibles sur place aujourd'hui. Plusieurs d'entre eux ont été rapportés à Vientiane pour y être conservés, notamment dans la galerie occidentale du That Luang (qui est ainsi devenu un musée) et au Vat Ho Phra Kèo. Il est devenu par ailleurs fréquent, en particulier depuis les années 1990, de découvrir, à l'occasion de travaux de construction, de nouveaux vestiges môns dans des endroits parfois inattendus. Ces pièces viennent alors compléter et affiner notre perception de ce passé ancien. Un autre rapport produit par Madeleine Giteau, faisant référence à quelques pièces d'art khmer conservées au Vat Ho Phra Kèo dans les années 1960, rendait également nécessaire qu'on portât un nouveau regard sur la possibilité d'une extension très septentrionale de la civilisation angkorienne ${ }^{6}$.

On reprendra dans ce long chapitre le principe de classement qui a été adopté pour les régions plus méridionales : la répartition en espaces caractérisés par une configuration physique facilement reconnaissable, en particulier les bassins hydrographiques. On peut déterminer, pour la plaine de Vientiane, plusieurs ensembles aux contours relativement précis, tous marqués par des positions de sites archéologiques bouddhiques à proximité immédiate de cours d'eau et surtout de confluents (cf. carte supra, p. 38).

\section{Le confluent de la Nam Ngum et de la Nam Lik}

Le site historique pré-lao le plus septentrional de la plaine de Vientiane semble être à Ban Pak Pot (ou Ban Nam Pot), sur les bords de la Nam Lik, à 2 km à peine du confluent

4. Le riche potentiel archéologique de la partie septentrionale du nord-est de la Thaïlande sera présenté dans une nouvelle étude sur l'espace môn-khmer ancien dans la vallée moyenne du Mékong.

5. P.-M. Gagneux, Les sites anciens de la plaine de Vientiane (VII'-IX' siècles), rapport pour le service des Monuments historiques du Laos, 1977. Ce document (non paginé) contient un avant-propos, 19 pages concernant les lieux où les vestiges étaient conservés in situ ou avaient été déplacés, 10 pages de réflexion sur les liens entre un supposé « art de Sikhottabong », l'art de Dvāravatī et l'art du Lān Xāng, 16 pages d'inventaire détaillant 105 pièces jugées mônes (M1 à M105), 16 pages contenant 81 croquis de ces pièces (très utiles pour retrouver leur lieu actuel), 6 pages d'index, 2 pages de bibliographie et 2 cartes. Il a été réédité presque intégralement dans Péninsule 11-12, 1985-1986, p. 27-83.

6. Madeleine Gitedu, Laos. Étude de collections d'art bouddhique, 17 décembre $1968-17$ mars 1969 , rapport de mission Unesco (distribution limitée), 69 pages +20 pages de photographies et d'illustrations. Ce document est en grande partie consacré aux pièces bouddhiques d'époque lao et ignore les pièces relevant du monde môn. 
avec la Nam Ngum, importante rivière descendant du plateau de Xieng Khuang. Les terres qui entourent ce confluent paraissent avoir constitué un foyer très important de culture mône, puisque si les vestiges y sont particulièrement concentrés, certains d'entre eux figurent également parmi les plus remarquables découverts au Laos. La raison de l'importance de cette région est d'abord purement géographique : le point de contact entre les deux rivières se trouve en effet dans la zone où se rejoignent les deux lignes de relief qui entourent la plaine de Vientiane. À l'ouest, dans une direction globalement nord-sud, le Say Phu Pha Daeng, que prolonge le Say Phu Pha Nang, touche le Mékong à une vingtaine de kilomètres en amont de la capitale lao. Orienté vers le sud-est, sur une distance de plus de $60 \mathrm{~km}$, le massif montagneux où culmine le Phu Khao Khuay rejoint quant à lui le fleuve bien plus en aval, à la limite de la province de Borikhamxai. La Nam Lik et la Nam Ngum, qui viennent respectivement du nord-ouest et du nord-est, se frayent d'ailleurs un passage dans l'une et l'autre de ces chaînes montagneuses. Les enquêtes n'ont pas encore révélé d'occupation mône au-delà de ces reliefs. Mais, à titre d'hypothèse, il n'est pas absurde d'imaginer un lien entre ces populations austro-asiatiques de la plaine de Vientiane qui se sont distinguées par l'érection de stèles et de piliers en pierre (sema), témoignant de l'adoption du bouddhisme, et la civilisation protohistorique qui a parsemé le plateau de Xieng Khuang de grandes jarres et de monolithes taillés dans la roche.

\section{Ban Pak Pot}

En 1976, à l'occasion d'un défrichement, des cultivateurs ont exhumé un sema sculpté portant l'image stylisée caractéristique du stūpa à proximité immédiate du confluent de la Nam Pot et de la Nam Lik. L'emplacement de la découverte nous a été précisé par une vieille habitante du village : il est situé dans un jardin, sur la rive gauche de la Nam Pot, à une centaine de mètres à peine de son lit. Il est utile de noter qu'il existe entre ce jardin et le cours d'eau une sorte de grand tertre ovale (aucune trace de matériaux de construction en surface), cerné par une espèce de douve de 8 à $10 \mathrm{~m}$ de large, dont le côté sud jouxte directement la Nam Pot. Cette particularité physique est repérable en photographie aérienne et aurait pu être négligée si le sema n'avait pas été trouvé en ce point. La pièce (hauteur : $140 \mathrm{~cm}$, largeur : $58 \mathrm{~cm}$ ), qui a été rapportée après 1976 dans la galerie occidentale du That Luang (fig. 1), présente un intérêt certain par sa facture originale - et il est difficile de penser qu'elle ait pu être totalement isolée. L'intérieur de l'image du stūpa est constitué de quatre (voire cinq) niveaux. Le premier peut être considéré comme une base ${ }^{7}$. Il est possible que le second niveau représente deux personnages en prière, car l'irrégularité des formes et le rétrécissement de leur partie supérieure empêche de les identifier à des pièces de soutènement. Ce sont bien des colonnes, fines, qui semblent en revanche apparaître au troisième niveau : elles soutiennent le niveau supérieur et entourent ce qui semble être une image de Buddha assis. Ce type iconographique d'un Buddha positionné au-dessus de deux personnages est fréquent dans les stèles historiées mônes.

\section{Ban Thin Kèo}

Ban Thin Kèo est un village établi au confluent de la Nam Lik et de la Nam Ngum, sur la rive gauche de la seconde, en face de Ban Thalat. Des vestiges ont été mis en évidence dans deux temples situés sur la berge, que $200 \mathrm{~m}$ à peine séparent. Il n'est pas impossible

7. La base proprement dite du sema, c'est-à-dire la partie devant être enterrée, de même que la frise horizontale sur laquelle pouvait reposer l'image du stūpa, ne sont malheureusement plus visibles - et il semble qu'elles manquaient déjà au moment de la découverte, puisqu'elles ne figurent pas sur le croquis de P.-M. Gagneux. 
que ces vestiges aient fait partie à l'origine d'un même lieu de culte. Un troisième temple, sis précisément en face du confluent, conserve peut-être également un témoignage ancien.

Le Vat Thin Kèo se trouve à $700 \mathrm{~m}$ en aval du confluent. Encore complètement ruiné et abandonné il y a une dizaine d'années, il laissait tout de même voir, autour des restes d'un autel en briques, deux des trois vestiges qui avaient été relevés par P.-M. Gagneux en 1976. Début 2011, le temple avait toutefois retrouvé des moines et des novices, après que furent construits plusieurs bâtiments dont une sãlā et des kuți. Sur une butte dominant la rivière, un nouveau $\operatorname{sim}$, de grande dimension, était en outre pratiquement achevé. Il semble par ailleurs que ce soit sur les ruines de l'autel qu'ait été érigé très récemment un petit stūpa dont la forme rappelle la flèche du That Luang, pratiquement accolé à la sālā. Cette dernière contient plusieurs vestiges archéologiques, parmi lesquels on reconnaît la grande image en grès du Buddha assis qui avait été notée par P.-M. Gagneux (M28). Elle était également identifiable en 2000 , mais cassée en deux fragments, la tête reposant à côté du tronc (dont toute la partie inférieure était alors enterrée). La statue est aujourd'hui à nouveau visible dans son état complet (fig. 2), puisqu'elle a été entièrement dégagée et que la tête (très abîmée) a retrouvé sa position initiale, sans être toutefois fixée. Des restes de stuc apparaissent nettement sur le corps : une couche de ce matériau semble avoir recouvert l'intégralité de l'image, comme c'est le cas pour beaucoup d'autres objets de culte très anciens qui ont étć rćutilisćs à l'ćpoque lao. Parmi les deux autres vestiges du Vat Thin Kèo signalés par P.-M. Gagneux, il en est un (M31) auquel il accordait une attention particulière et qui est maintenant conservé dans une vitrine de la galerie occidentale du That Luang. Il s'agit d'une tête de " divinité » (en fait, très probablement le Buddha) recouverte de plusieurs couches de stuc, mais dont le noyau initial, en grès, est rendu visible au niveau du visage (fig. 3). La pièce est assez étonnante, tant par la qualité de sa facture que par son apparence complexe (juxtaposition des enduits). Elle contraste singulièrement avec la troisième pièce signalée en 1976 et retrouvée sur le site en 2000 : le fragment d'un Buddha assis adossé à une stèle en grès, dont la tête et tout le côté droit manquaient (M29). L'image paraissait tellement fruste qu'il ne semblait s'agir que d'une ébauche. Or, à l'occasion des travaux qui ont été effectués en 2009 pour l'érection des $k u t i i$ et de la sālă, trois nouvelles pièces (au moins) en grès ont été mises au jour - et l'on trouve parmi elles le fragment droit d'un torse de Buddha adossé à une stèle qui s'ajuste parfaitement au vestige mentionné précédemment. Si la partie supérieure comprenant la tête n'a pas encore été retrouvée, il s'avère en tout cas que, dans son état initial, la statue était de meilleure facture que ce qu'on pouvait penser, puisque le modelé du côté droit a bien été préservé. L'enfouissement prolongé de celui-ci l'a manifestement protégé des détériorations qu'ont dû subir la base et le côté gauche, dont on s'est peut-être longtemps servi pour l'aiguisage des couteaux. La deuxième pièce récemment mise au jour appartient sans conteste aux vestiges môns de la province de Vientiane, mais elle est pourtant unique, tant par son traitement général que par son iconographie. Il s'agit d'une borne (beaucoup plus que d'une stèle) sur laquelle est sculpté en bas-relief un personnage masculin (l'identification au Buddha n'est pas tout à fait certaine) debout - dont la tête a disparu - et qui est représenté dans une attitude particulière, puisque son bras droit est replié vers l'avant, alors que son bras gauche semble reposer sur son ventre (fig. 4). Si la facture est très maladroite, on ne peut manquer pour autant d'être intrigué par l'ensemble de cette grande composition (hauteur : $117 \mathrm{~cm}$, largeur : $33 \mathrm{~cm}$ ) où se mélangent des éléments qui appartiennent autant au Buddha debout qu'au Buddha assis. La troisième pièce retrouvée en 2009 est une tête de Buddha d'une hauteur de $25 \mathrm{~cm}$. Elle ne paraît pas correspondre à la partie manquante de l'image précédente (traitement en ronde-bosse) 
et elle est probablement issue d'un corps qu'on n'a pas encore retrouvé. Bien que très abîmée, notamment au niveau du visage, elle fait partie des plus belles pièces de ce type qui ont été mises au jour dans la plaine de Vientiane. En 2000, les ruines du Vat Thin Kèo conservaient encore deux autres vestiges en grès - un capuchon de nāga et un torse de Buddha - qui semble-t-il n'avaient pas été vus par P.-M. Gagneux et qui pourraient donc avoir été découverts après 1976. Ils n'ont plus été retrouvés en 2011 . Une grande stèle non achevée ou très abîmée, dressée entre la sālā et les kutii, constitue probablement un sema non historié.

Le Vat Okat de Ban Thin Kèo était de reconstruction récente en 1968. Le torse masculin et le Buddha assis en grès (sans tête) vus par P.-M. Gagneux ne semblent plus y être conservés, mais le croquis qui est donné du premier montre qu'il était de même type que les images TL I/31 et TL I/41-1 visibles au That Luang (cf. infra). Le temple abrite en revanche deux grands monolithes (plus de $120 \mathrm{~cm}$ de long) que P.-M. Gagneux qualifiait de « linga en pierre, de section elliptique et de facture très réaliste ». À l'époque, seul l'un d'eux (M74) était totalement dégagé, tandis que l'autre (M75) émergeait verticalement du sol dans une position inversée (le bas en haut) qui renforçait son aspect phallique. Le côté " réaliste » était encore accentué par la présence, sur chacune des pièces, d'un bandeau (double ou simple) délimitant une extrémité à la forme suggestive (fig. 5 ) $^{8}$. La confusion n'est plus guère possible aujourd'hui : ces deux sema - puisque c'est bien de cela qu'il s'agit - sont désormais dressés dans leur position normale sur un socle de béton, qui laisse tout de même voir une partie de leur base (réduite et donc moins suggestive) avec leur bandeau. Le traitement plus complet d'un de ces bandeaux, divisé en deux frises ornées de boutons, de même que la présence de faces plus plates, prouvent bien que l'identification à des linga était erronée. Le temple conserve également deux Buddha debout : il s'agit peut-être de sculptures anciennes qu'on a recouvertes avec de nouveaux matériaux (ciment, stuc, peinture). Cette observation s'applique en particulier à une grande image au traitement plastique assez grossier qui est adossée à une stèle d'aspect totalement moderne. Comme rien n'indique que les sema môns du Vat Okat ont été découverts in situ, il est possible qu'ils aient été rapportés du Vat Thin Kèo tout proche, dont on a pu vérifier encore récemment que le sol recelait une richesse en vestiges certaine.

Le Vat Ongteu est situé juste en face du confluent de la Nam Ngum et de la Nam Lik, à un endroit qui devrait en principe lui conférer une importance toute particulière. Le potentiel archéologique du site n'a cependant pas encore été vérifié, sauf peul-être avec le fragment très abîmé d'une tête de Buddha adossée à une stèle, qui semble bien être en grès rose. La pièce étant confinée à l'arrière du Buddha principal (moderne), il est difficile de dire s'il s'agit de la partie manquante du Buddha assis du Vat Thin Kèo.

\section{Ban Thalat}

Ban Thalat est un village qui a connu un fort développement à partir de la fin des années 1960, avec la construction du premier barrage hydraulique au Laos. L'importance archéologique de l'endroit a été mise en évidence une première fois en octobre 1968, de façon particulièrement éclatante, avec la découverte fortuite d'une très belle image du Buddha et d'une inscription en langue mône qui ont pu être datées du viII ${ }^{\mathrm{e}}$ siècle environ

8. Ce caractère réaliste, encore plus évident avec d'autres pièces, comme l'un des sema de Ban Na Sone (cf. infra), nous avait nous-même d'abord induit en erreur, puisque nous avions « reconnu » huit linga dans la province de Vientiane. Cf. M. Lorrillard, « Pour une géographic historique du bouddhisme au Laos », dans Yves Goudineau \& Michel Lorrillard (éd.), Recherches nouvelles sur le Laos, Études thématiques $n^{\circ} 18$, Vientiane / Paris, EFEO, 2008, p. 124 et fig. 15 p. 175. 
(fig. 6-7) ${ }^{9}$. Les deux pièces ont été trouvées ensemble au même endroit, au sommet d'une petite colline où l'on a d'abord construit un camp militaire, puis un temple : le Vat Pho Seng Arun.

C'est sur cette même colline qu'ont été mis au jour le 8 février 2004 quatre sema dont un, assez abîmé, porte l'image stylisée du stüpa avec intégration du motif du kumbha (pot à eau rond), caractéristique fréquente sur les vestiges de ce type dans le nord-est de la Thaïlande ${ }^{10}$. Cette particularité iconographique qui consiste à associer en un même ensemble deux symboles de bon augure et deux formes distinctes (une pointe posée sur une base circulaire) se retrouve dans quelques autres sites de la plaine de Vientiane (cf. infra), mais jamais de façon aussi prononcée ${ }^{11}$.

Il semble que ce soit quelques jours plus tard que cinq autres sema furent exhumés, dont un était également historié ${ }^{12}$. Il s'agit probablement de celui qu'on peut voir aujourd'hui dressé devant le sanctuaire : une face montre l'image du stūpa-mais dans son traitement le plus simple, en forme d' «épine »-alors que l'autre face, « restaurée » à grand renfort de ciment, paraît avoir également présenté une image qui est maintenant recouverte.

Le site de Ban Thalat est d'un point de vue archéologique le plus significatif de tous les sites môns identifiés au Laos, puisqu'on y a retrouvé les trois types de vestiges qui prouvent le mieux leur appartenance à cette culture : une inscription en écriture et en languc môncs, unc grande image du Buddha en grès dont la facture est tout à fait caractéristique, et enfin des bai-sema avec image du stüpa.

Il n'est pas inutile de préciser que le Vat Pho Seng Arun de Ban Thalat et le Vat Ban Thin Kèo se font pratiquement face - de part et d'autre de la Nam Ngum - et que $600 \mathrm{~m}$ à peine (à vol d'oiseau) les séparent.

\section{Le confluent de la Nam Ngum et de la Nam Cheng}

La Nam Cheng est une petite rivière qui prend sa source sur les contreforts de la chaîne Say Phu Pha Nang, à quelque $400 \mathrm{~m}$ d'altitude. Elle suit d'abord une direction nord-est, puis oblique vers le sud-est, à l'endroit où elle est franchie par la route $\mathrm{n}^{\circ} 13$ (nord), jusqu'à sa jonction avec la Nam Ngum. Si son débit n'est pas important, il semble en tout cas - au vu de la répartition de certains vestiges - qu'elle ait constitué une voie de pénétration vers l'ouest pour certaines populations. Celles-ci se sont d'abord concen-

9. P.-M. Gagneux, « Vers une révolution dans l'archéologie indochinoise : le Buddha et la stèle de Thalat, Vientiane ", Bulletin des Amis du Royaume Lao 7-8, 1972, p. 83-105 ; Emmanuel Guillon, «Recherches sur quelques inscriptions môn, I. Traces de l'influence môn dans la plaine de Vientiane (Laos) ", BEFEO 61, 1974, p. 339-348. Christian Bauer a montré récemment (communication personnelle, 2011) qu'une transcription et une lecture légèrement différentes de l'inscription pouvaient être effectuées. Malgré la découverte d'autres épigraphes mônes dans la plaine de Vientiane ces dernières années (cf. infra), celle de Ban Thalat reste à ce jour la plus importante, dans la mesure où elle présente un texte complet dont le sens général ne pose pas de problème particulier : elle commémore l'œuvre méritoire d'un seigneur qui fait don à un sanctuaire d'esclaves et de bovins. E. Guillon donne dans son article une description détaillée du Buddha de Ban Thalat, qui est également la plus belle image mône trouvée jusqu'à présent dans cette partie septentrionale du bassin inférieur du Mékong.

10. S. A. Murphy, The Buddhist Boundary Markers..., op. cit. On pourra aussi parler à propos de ce pot de pürnaghața ou de pürnakalaśa, noms qui expriment alors les notions d'abondance et de prospérité.

11. Le fait qu'un sema aussi typé soit associé au Buddha et à la stèle de Ban Thalat, qui sont euxmêmes des vestiges tout à fait exceptionnels, n'est certainement pas fortuit. Des études plus poussées permettront peut-être d'établir un lien particulier avec un site du nord-est de la Thaïlande.

12. Pour les conditions relatives à ces découvertes, cf. Anna Karlström, Preserving Impermanence. The Creation of Heritage in Vientiane, Laos, Studies in Global Archaeology 13, Uppsala, Uppsala Universitet, 2009, p. 131-133. 
trées autour du confluent des deux rivières, entre Ban Muang Kao, à $2 \mathrm{~km}$ en amont, et Vieng Kham, à environ $5 \mathrm{~km}$ en aval. Vers l'intérieur, le site rupestre de Vang Sang a dû constituer un lieu privilégié pour la pratique religieuse.

\section{Ban Muang Kao}

Le village de Ban Muang Kao n'est qu'à une dizaine de kilomètres de Ban Thalat, en aval (au sud), mais la rivière se fraye entre ces deux points un passage dans un petit massif qui, aujourd'hui encore, est pratiquement inoccupé. Une large couverture végétale recouvre sur les deux rives cette zone intermédiaire. Il n'est pas interdit de croire que des vestiges pourront y apparaître lorsque l'activité humaine s'y sera davantage développée.

P.-M. Gagneux soulignait en 1977 l'ancienneté de Ban Muang Kao, rappelée par son nom même (« village du muang ancien »). Trois sites ont été mis en évidence en 1973, mais la répartition des vestiges a quelque peu changé depuis cette date.

Le Vat Keng Noy Kao, «monastère abandonné, situé sur une petite colline, dominant la rivière, à $2 \mathrm{~km}$ environ en amont du centre du village ${ }^{13} »$, abritait il y a une quarantaine d'années deux vestiges intéressants : un sema avec figuration du stüpa sur ses deux faces, et ce qui était présenté comme un linga. Le premier est aujourd'hui conservé dans la galerie occidentale du That Luang (fig. 8). La face qui reste visible (l'autre est pratiquement collée au mur) présente sans doute l'image de stūpa la plus élaborée de toute la plaine de Vientiane (même si la représentation reste relativement sobre). Le motif du kumbha y est visible (position centrale) et l'on retiendra en particulier l'exécution soignée de la base, avec ses divers types de frises. La qualité de cette stèle prouve à elle seule l'importance que pouvait avoir le site. De la seconde pièce, qui avait été réutilisée dans les années 1970 pour figurer la pointe terminale d'un mausolée funéraire, P.-M. Gagneux nous disait qu'il s'agissait d'un linga de section octogonale terminé par un gland ogival. La description correspond à ce qui est visible aujourd'hui, mais le vestige est recouvert d'une couche de peinture dorée, et probablement d'un enduit qui a accentué l'aspect rond - terminé par une petite pointe - de la partie supérieure. Pas plus que pour les vestiges du Vat Okat et quelques autres pièces dont nous parlerons plus loin, l'identification au symbole phallique ne doit cependant être retenue. Le « gland » était certainement la base d'un sema non historié.

Le Vat Muang Kao, situé au centre du village, était de construction récente au début des annćcs 1970. Aujourd'hui, on ne prêterait pas une trop grande attention à l'ensemble des Buddha assis dorés qui reposent sur son autel, si P.-M. Gagneux ne nous avait appris que neuf d'entre eux furent rapportés des ruines du Vat Mün Xu et qu'ils sont faits du même grès rose que le Buddha debout de cet endroit, emporté au That Luang. La facture générale semble assez grossière à cause des proportions qui ne sont pas réellement respectées et du lissage de tous les détails par l'épaisse couche qui recouvre les images (certaines d'entre elles avaient déjà été retouchées au ciment lorsqu'elles furent signalées en 1977). Les mains de deux de ces Buddha sont dans une position qui n'est pas habituelle : elles sont croisées sur les mollets ou traitées à l'inverse de la position en bhümisparśamudrä (c'est alors la main gauche qui touche le sol). Elles sont de taille assez grande - sept approchent ou dépassent $1 \mathrm{~m}$ de hauteur - et l'on peut reconnaître, en essayant de faire abstraction de leur " maquillage », un type qui est bien représenté dans la collection de la galerie occidentale du That Luang. 
Le peu qui subsiste aujourd'hui du Vat Mün Xu se trouve un peu plus en aval, en face de l'actuel village de Ban Pak Kagnung, et à 1200 mètres au nord du confluent de la Nam Cheng. Le temple, qui se réduisait déjà aux ruines d'un autel il y a une quarantaine d'années, n'était plus repérable que par un petit groupe de vestiges en 2000 , et n'existe plus guère aujourd'hui que dans le souvenir qu'en conservent certaines personnes - puisqu'il est maintenant remplacé par le jardin d'une propriété privée. P.-M. Gagneux avait relevé dans son périmètre trois pièces en grès. La première était une grande image $(178 \mathrm{~cm}$ de hauteur) de Buddha debout adossée à une stèle. Cette statue, très abîmée, est maintenant conservée dans la galerie occidentale du That Luang sous le $n^{\circ}$ TL I/10 (fig. 9) ${ }^{14}$. Il en est de même pour la troisième pièce mentionnée (TL $\mathrm{I} / 12$ ), un grand «ling $a$ » de section circulaire avec une base de section carrée, qui fut trouvé à proximité, " dans la forêt » (fig. 10). L'identification de P.-M. Gagneux est-elle ici plus juste ? La pureté de forme de cette pièce - qu'on pourrait éventuellement rapprocher de celle des linga angkoriens très stylisés - ne permet pas de formuler un jugement définitif. En raison de sa hauteur $(192 \mathrm{~cm})$, il faudra toutefois la comparer plutôt à quelques grands sema octogonaux qu'on trouve dans la proche région (cf. infra). Par sa facture, elle est aussi très proche d'une autre pièce conservée au That Luang, de provenance apparemment inconnue (ancien numéro d'inventaire : 21-163/1), qui n'est peut-être que la partie supérieure d'un vestige beaucoup plus grand. P.-M. Gagneux avait par ailleurs repéré au Vat Mün Xu une stèle très érodée, de forme ogivale, portant la représentation stylisée d'un stüpa sur l'une de ses faces. Des trois pièces signalées en 1977, celle-ci est la seule qui soit restée à proximité directe de l'ancien temple, à terre, à quelques mètres même de la rivière. Elle voisine encore avec la base émergeant du sol d'un autre sema, partie que délimitent bien deux bandes horizontales et que P.-M. Gagneux aurait certainement identifiée au sommet d'un linga. Il serait souhaitable que ce vestige soit totalement exhumé.

En 2000, une pièce analogue (avec une frise ornée de boutons), un sema portant sur l'une de ses faces l'image stylisée du stūpa et deux autres bornes aux formes plus imprécises étaient encore visibles sur le même terrain. Les deux premiers vestiges ont été emportés au tout récent Vat Tha Pho Xay, à $400 \mathrm{~m}$ à l'est (ils ont alors été éloignés du bord de la rivière), et les deux autres semblent avoir été dispersés sans grande attention (c'est peut-être l'un d'eux qui se dresse aujourd'hui à proximité, au bord de la route). Remarquons que par leur facture, les deux parties visibles de sema / linga du Vat Mün $\mathrm{Xu}$ ressemblent fort aux bases des pièces complètes qui sont conservées au Vat Okat de Ban Thin Kèo.

\section{Ban Don Koat}

Le temple de Ban Don Koat, riverain de la Nam Ngum et situé à 3,5 km au sud du Vat Mün Xu (2,2 km en aval du confluent de la Nam Cheng), a récemment révélé la présence de six stèles en grès, dont quatre ne consistent plus qu'en de gros fragments. Toutes paraissent avoir la même origine et ressemblent aux sema non historiés môns, comme il s'en retrouve en amont et en aval. Trois de ces stèles portent des inscriptions en langue et écriture lao inédites (datées apparemment de 1520, 1574-1575 et 1631), mais cette caractéristique ne doit pas nous tromper sur l'origine des supports, car d'autres

14. La stèle à laquelle s'adosse la statue présente une particularité : elle n'est pas totalement plate et sa partie supérieure se prolonge au-dessus de la tête, à la manière d'un capuchon. D'ailleurs, de chaque côté de celui-ci, on peut voir les restes d'une tête de nāga, dont le corps apparaît également à l'arrière. Si les avant-bras du Buddha manquent, il semble bien qu'ils étaient tous les deux repliés vers les aisselles et qu'ils effectuaient soit le geste de l'abhayamudrā, soit celui de la vitarkamudrā. 
exemples - en particulier les deux stèles inscrites de Vieng Kham (cf. infra) - montrent clairement que les plus anciennes inscriptions lao ont été très souvent gravées sur des blocs de grès de facture mône, dont la sculpture était antérieure de plus de cinq siècles.

\section{Ban Thin Yung}

Une visite, en 2004, du temple de ce village situé au sommet d'un méandre étroit de la Nam Ngum, à $2,5 \mathrm{~km}$ en aval de Ban Don Koat, avait montré la présence, émergeant du sol, du sommet d'un bloc de grès de forme arrondie sur lequel était gravé de façon maladroite (et sans doute à une date récente) un faux disque horoscopique ${ }^{15}$. Ce sommet semblait être en fait la base d'un sema du type de ceux qu'on retrouve à proximité. Il doit s'agir de l'extrémité à peine visible aujourd'hui de l'un des deux blocs de grès qui ont été coulés dans la chape de béton entourant le sanctuaire depuis quelques années. L'hypothèse d'une identité mône de ce sema est renforcée par la présence dans le temple de deux images de Buddha assis en position de méditation - adossés à une stèle -, dont l'ancienneté est certaine. La plus grande $(98 \mathrm{~cm}$ de hauteur) montre une coiffure qui pourrait faire penser à une production khmère, mais elle demeure par sa facture générale associée aux images mônes, auxquelles se rattache parfaitement la seconde statue.

\section{Ban Vieng Kham}

Le nom de Vieng Kham s'est conservé dans les chroniques lao les plus anciennes, et même dans l'épigraphie de Sukhothai (inscription de Rama Khamhaeng, 1292). Il est alors associé à celui de Vieng Chan (Vientiane), comme si ces deux muang avaient longtemps eu une destinée commune ${ }^{16}$. Le site n'a cependant pas connu le même développement (à l'époque contemporaine, c'est Ban Keun, situé pratiquement en face, qui est devenu un bourg important), et il ne diffère guère aujourd'hui d'autres petits villages. Il est même absent de l'inventaire effectué par P.-M. Gagneux ${ }^{17}$, qui ne semble pas avoir noté que les deux inscriptions lao retrouvées dans le «Vat Kao »-ruines d'un ancien temple - et rapportées à Vientiane sont en fait gravées sur des grands sema de facture typiquement mône (fig. 11) ${ }^{18}$. En février 2004, lorsqu'Anna Karlström effectua des recherches archéologiques sur le domaine de cet ancien sanctuaire, d'autres sema, dont deux portaient sur leurs deux faces l'image stylisée du stüpa, étaient toujours visibles en surface. Un sondage permit d'excaver complètement une pièce dont seul le sommet apparaissait jusqu'alors et qui se révéla de belle taille (plus de $1,50 \mathrm{~m}$ ), avec une forme

15. Observations faites à partir de photographies prises et communiquées gracieusement par Anna Karlström.

16. M. Lorrillard, «Vientiane et le Mékong : situation de la ville dans l'espace régional et la longue durée ", dans Sophie Clément-Charpentier, Pierre Clément, Charles Goldblum et al. (dir.), Vientiane, architectures d'une capitale. Traces, formes, structures, projets, Paris, éditions Recherches / Ipraus, 2010 , p. $33-50$.

17. Cet auteur mentionne cependant ce site comme un ancien sanctuaire lao, dans Pierre-Marie Gagneux \& Georges Pfeiffer, Reconnaissances archéologiques dans la plaine de Vientiane, document ronéotypé de la Mission d'enseignement et de coopération culturelle, 1970, 29 pages + 2 cartes, p. 17.

18. Le premier de ces sema, conservé (en deux fragments) au Vat Ho Phra Kèo de Vientiane depuis 1967, porte sur ses deux faces une inscription en lao qui indique, entre autres données, les années 1549 et 1561-1562. Le second, qui fut transporté au That Luang en 1976 ( $\mathrm{n}^{\circ}$ TL I/13), montre une facture épaisse mais régulière, et ne porte sur la partie supérieure d'une de ses faces que quelques mots lao indiquant une date : apparemment le 29 novembre 1601 (il s'agissait d'un jeudi, mais c'est un mercredi qui est mentionné). Le traitement de la base des deux blocs ne laisse aucun doute sur l'ancienneté de la sculpture, bien antérieure au $\mathrm{XVI}^{\mathrm{e}}$ siècle. 
hexagonale et une base de facture typiquement mône marquée par une frise ${ }^{19}$. Un autre sondage révéla un nouveau sema, mais de taille moins importante, à deux faces plates et non historiées, qui fut emporté à Vientiane pour y être exposé. Le site n'a plus rien révélé d'autre, même si des travaux y ont été effectués depuis quelques années pour édifier un nouveau temple. Il est possible, comme le suggère A. Karlström, que Ban Vieng Kham ait été protégé par une levée de terre, bien que la photographie aérienne ne puisse l'attester de façon certaine ${ }^{20}$.

Ban Vieng Kham est pour l'instant - parmi les sites môns directement riverains de la Nam Ngum - celui qui se trouve le plus en aval (seuls $20 \mathrm{~km}$ le séparent pourtant du confluent de la Nam Lik). Il fait partie d'un ensemble où nous avons identifié au moins neuf lieux d'occupation. La rivière étendant encore son cours méandreux sur près de $100 \mathrm{~km}$ avant d'atteindre le confluent du Mékong, il ne serait pas étonnant que d'autres sites puissent être découverts sur ses berges à la fois plus méridionales et orientales. Le site protohistorique de Lao Pako, situé à plus de $50 \mathrm{~km}$ en aval, a montré des vestiges très différents (céramique, bronze, pierre taillée, etc.), significatifs d'un contexte d'occupation distinct : il s'agit ici d'un lieu de vie et de mort (sépultures), et non pas d'un lieu de culte bouddhique ${ }^{21}$. Mais le fossé culturel et chronologique existant entre ces types de sites n'est pas forcément aussi large qu'on pourrait le croire.

\section{Vang Sang}

Le site de Vang Sang est riverain de la Nam Cheng, mais une douzaine de kilomètres (à vol d'oiseau) le séparent du confluent avec la Nam Ngum. La petite rivière permet de le lier directement au groupe des sites qui précèdent. Du point de vue de la latitude, il est à peu près à la même hauteur que Ban Muang Kao - et il n'est pas plus distant de ce point que ne l'est Ban Pak Pot.

Vang Sang est le site ancien de la plaine de Vientiane qui a été à la fois le plus tôt et le plus fréquemment mentionné, puisque des descriptions apparaissent chez Charles Batteur, Suzanne Karpelès et Pierre-Marie Gagneux ${ }^{22}$. Il se signale principalement par la présence de ses groupes de Buddha - certains exceptionnels par leur taille - sculptés en bas-relief dans une paroi rocheuse. En cela, il peut comme Dan Sung (cf. infra) être qualifié de « site rupestre ».

Toutefois, les images qui sont visibles aujourd'hui diffèrent sensiblement de celles qui ont été sculptées à l'origine, car des retouches importantes ont été faites au milieu du $\mathrm{xx}^{\mathrm{e}}$ siècle. Les différents enduits (ciment, peinture) qui ont été ajoutés tendent à estomper l'aspect du modelé original, en particulier sur les visages, à tel point que l'ancienneté de certains de ces vestiges est devenue à peine reconnaissable. Dans la mesure où les rapides

19. A. Karlström, Preserving Impermanence..., op. cit., p. 168.

20. A. Karlström, Preserving Impermanence..., op. cit., p. 159. Ce sont des dépressions identifiées à des fossés / douves («moat »), et non des murs, qui semblent avoir été reconnues. Le tracé que donne l'auteur correspond sans doute à celui d'un ancien méandre.

21. Anna Källén, And Through Flows the River: Archaelogy and the Pasts of Lao Pako, Studies in Global Archaeology 6, Uppsala, Uppsala Universitet, 2004.

22. Charles Batteur, « Sculptures rupestres au Laos », BEFEO 25/1, 1925, p. 203-204 ; Suzanne Karpelès, «Chronique : Laos», BEFEO 33, 1933, p. 1144 (premières indications sur le site) - « Les grottes sculptées de la province de Vientiane, vestiges de l'art de Lavapuri », Bulletin des Amis du Laos 4, 1949, p. 141-148 - "Les grottes sculptées de la province de Vientiane », France-Asie 118-120, 1956, p. 770-772 (résumé non illustré de l'article précédent) ; P.-M. Gagneux, Les sites anciens..., op. cit. 
témoignages publiés jusqu'à présent sur ce site entretiennent eux-mêmes une certaine confusion, il est utile, ici, de revenir sur certaines descriptions.

Ch. Batteur est le premier à signaler le site de Vang Sang - alors dénommé « anfractuosité de Nong Phin " (nom d'un village supposé proche) - dont il écrit qu'« il [lui] a donné l'impression - sujette à vérification en raison de la rapidité de [s]a visite - d'être le reste d'une grotte, d'une anfractuosité ou d'une fosse naturelle, en partie aménagée par l'homme et qui se serait presque totalement écroulée. Il subsiste des parois qui semblent être comme deux des angles d'une immense salle, ou le fond ou le côté d'un vaste et grossier temple rupestre, sans plafond ${ }^{23}$ ». Le premier plan qu'il dresse en juillet 1923 à l'occasion de cette visite (fig. 12), légèrement différent et sans doute plus explicite que le plan publié avec sa note - renforce effectivement cette impression de « salle ", à laquelle est donnée de façon schématisée une forme rectangulaire. L'un des grands côtés (nord) est alors ouvert sur l'extérieur, tandis que l'autre (sud) forme la masse de la paroi (P - P'), rompue en deux endroits - de façon transversale -, créant ainsi trois ensembles distincts d'une hauteur de 6 à $8 \mathrm{~m}$. Les petits côtés sont représentés à l'est par un angle droit de la paroi qui s'avance vers le nord sur une dizaine de mètres (avant de s'infléchir à nouveau vers l'est où il se prolonge sur une bonne distance) et à l'ouest par une autre avancée, un peu plus courte, qui constitue cependant la seconde tranche détachée du massif, laquelle révèle à l'intérieur de celui-ci une étroite et profonde fissure formant une sorte de corridor. Le terme « grotte » ne peut cependant être employé pour l'ensemble (« abri-sous-roche » ne conviendrait pas davantage), tant il est certain que le site ne ressembla jamais à une vaste cavité naturelle dans laquelle on aurait pu s'enfoncer. L'emploi du mot " fosse ", signifiant qu'on aurait eu accès à l'endroit par une partie en surplomb, paraît encore plus erroné. S'il est certain que cette extrémité de massif rocheux a été fracturée en trois ensembles, il est très imprudent de penser que ce bouleversement géologique se serait produit après un aménagement du lieu par l'homme ${ }^{24}$. On peut supposer par ailleurs, contrairement à l'opinion de Ch. Batteur, qu'il y eut là un « plafond », ou en tout cas un ensemble de structures destinées à couvrir une partie du sanctuaire. Leur existence est prouvée par la présence à différents endroits de la paroi, y compris dans sa prolongation vers l'est, de plusieurs mortaises de forme rectangulaire dans lesquelles des «poutres » devaient s'encastrer.

La première paroi sculptée de Vang Sang fait face au nord. Les cinq Buddha qu'on pcut observer en arrivant par le chemin habituel se trouvent dans l'angle sud-ouest, correspondant à la partie $\mathrm{B}$ du plan de $\mathrm{Ch}$. Batteur. Celui-ci leur donne à tous une attitude identique, alors que $\mathrm{S}$. Karpelès remarque, à juste titre, que l'image centrale, plus grande que les autres, effectue de la main droite le geste de l'argumentation. Les quatre autres statues sont en position de méditation - les deux mains reposant dans le giron - et semblent avoir été exécutées selon le même modèle. Des photographies anciennes montrent que c'est la cinquième image (e), isolée sur la partie détachée de la paroi, qui

23. Ch. Batteur, « Sculptures rupestres au Laos », op. cit. p. 203.

24. Ch. Batteur rapporte dans sa fiche de terrain datée de juillet 1923 : « Le groupe de statues B était un tout symétrique [...]. Un accident (?), en rompant le rocher, en $x$ [lettre indiquant la fissure sur le plan], a écarté les statues d et e ». Rien n'empêchait cependant le sculpteur de réaliser son œuvre après la rupture de la paroi, c'est-à-dire de tailler d'abord les quatre premiers Buddha $(a, b, c$ et d) et de continuer sur la paroi opposée avec un cinquième Buddha (e) en regard. Il est au contraire assez. étonnant de penser que l'énorme brèche aurait pu se produire postérieurement à la sculpture - d'autant plus qu'elle se trouve précisément à la limite entre le quatrième et le cinquième Buddha, et qu'elle n'a altéré aucune des images. 
a le plus souffert des affres du temps au cours de ces dernières décennies (fig. 13-14). La finesse de son visage et de sa chevelure a disparu. Ces cinq Buddha ne semblent pas avoir été l'objet des mêmes restaurations que le groupe des images situées dans l'autre angle, car ils ont échappé aux enduits de ciment et n'ont été recouverts que de peinture. On peut encore noter, à la suite de $\mathrm{S}$. Karpelès, que la quatrième image $(\mathrm{d})$, en bordure de la grande fissure, paraît moins achevée.

Les informations que livre $S$. Karpelès pour les images de l'angle sud-est, groupe le plus intéressant, sont à considérer avec plus de prudence. Elle se trompe en plaçant sur la face orientale (qu'elle présente d'ailleurs comme occidentale) de la paroi trois figures sculptées, "deux petites, chacune dans une niche, et une grande de trois mètres de haut, assises à l'indienne et faisant le geste de l'argumentation ». Cette paroi ne compte en effet que deux images, et toutes deux sont très grandes et placées dans une niche (fig. 15). Celle qui est à droite, avec ses $4 \mathrm{~m}$ de hauteur, dépasse l'autre d'une cinquantaine de centimètres seulement. Sa niche, par ailleurs, présente de chaque côté des motifs divers dont l'identification n'est pas forcément aisée (fig. 16). S. Karpelès évoque à leur propos " au centre [...], deux crocs à éléphants, au-dessus desquels se trouvent deux fleurs de lotus ; en haut et en bas on reconnaît deux bannières, deux feuilles de lotus, deux écrans dentelés pareils à une feuille de l'arbre de l'Illumination, dont l'un est pourvu d'un long manche ; sous l'oriflamme du bas se trouvent cinq traits verticaux », mais il ne s'agit là que des éléments placés à droite du plus grand Buddha, et il semble qu'un écran dentelé ait été compté en trop, alors que manque une figure en forme de flamme. À gauche du même Buddha, se trouveraient alors deux fleurs de lotus, deux feuilles de lotus, des bannières et peut-être les crocs à éléphants. Il est très difficile aujourd'hui de reconnaître l'identité précise de ces motifs - toutefois certains d'entre eux, par leur forme, pourraient aussi correspondre à des insignes valorisants qu'on retrouve dans l'iconographie des bas-reliefs du Cambodge angkorien, comme les chasse-mouches, les éventails et les parasols ${ }^{25}$. L'unique autre exemple de ce type d'élément décoratif se trouve à Dan Sung (cf. infra).

$\mathrm{S}$. Karpelès rapportait que la figure sise à droite du grand Buddha était assez endommagée. Les photographies anciennes montrent que c'est surtout la grande image ellemême qui était abîmée, en particulier sa partie inférieure, puisque les jambes croisées avaient pratiquement disparu. Elles furent apparemment refaites en ciment vers 1957, et les « restaurateurs » en profitèrent pour remodeler d'autres parties, dont les visages de plusieurs images. Les enduits et la peinture modifièrent ainsi les expressions de certaines statues en leur ouvrant les yeux (ils étaient à l'origine clos, paupières baissées). Cette altération des formes est évidente sur les deux grands Buddha en vitarkamudrā, mais elle est encore plus flagrante sur les deux images assises en position de méditation (fig. 17-18) appartenant au petit groupe (c) de trois statues sculptées sur une roche qui s'est apparemment détachée de la falaise. La restauration moderne a ici complètement effacé le caractère très typé des visages dont les traits étaient bien marqués (tel l'aspect charnu des lèvres), rappelant des images de facture khmère. À gauche de ces deux Buddha figure une image debout dont l'avant-bras, brisé, montre tout de même que la main était levée : celle-ci tenait une tige avec une fleur de lotus reposant sur le buste, détail noté par $S$. Karpelès et qui est parfaitement visible sur une photographie ancienne - mais qui n'est plus identifiable aujourd'hui. La paroi révèle aussi, en plus des Buddha sculptés en basrelief, une petite image d'éléphant qui ne semble avoir été relevée ni par Ch. Batteur ni

25. Dominique Soutif, Organisation religieuse et profane du temple khmer du vIr au xIIr siècle, thèse de doctorat, Paris III, 2009, vol. I, p. 209-217, et vol. III, pl. XVII et XLVI. 
par S. Karpelès. Le caractère très particulier de cette figure, qui est sans doute à l'origine du nom du site ("Vang Sang " peut être traduit par " enclos des éléphants »), ne permet pas d'affirmer qu'elle doive être rattachée à la même époque que les Buddha. Entre les deux grandes images sont d'ailleurs gravés trois caractères qui leur sont en tout cas bien postérieurs : il s'agit du chiffre 928 en écriture lao, lequel correspond très certainement à une année de la petite ère (1566-1567 de l'ère chrétienne) et à une période - le règne de Setthāthirāt - où bien d'autres exemples montrent que des sites et des vestiges môns furent respectivement réoccupés et réutilisés.

\section{Ban Nong Khon}

Le village de Ban Nong Khon est situé à $8 \mathrm{~km}$ au sud-ouest de Vang Sang et à $14 \mathrm{~km}$ à l'ouest de la Nam Ngum, à peu près à la même latitude que Ban Thin Yung. La rive la plus proche de la Nam Cheng se trouve à $6 \mathrm{~km}$ à l'ouest. La position de Ban Nong Khon, où plusieurs sema môns ont été retrouvés, ne peut donc être comparée absolument à celle de tous les sites mentionnés précédemment, puisque ceux-ci étaient systématiquement installés au bord d'une rivière. Si la proximité de Vang Sang n'avait été établie, on l'aurait comparé davantage aux « sites de terre " groupés autour de Dan Sung, à une trentaine de kilomètres au sud. Il est toutefois fort probable que Ban Nong Khon ait été l'une des premières étapes d'un axe de communication terrestre reliant l'ensemble des sites " ripuaires » (Nam Lik, Nam Ngum, Nam Cheng) aux communautés plus méridionales, et même aux communautés occidentales établies derrière l'imposante barrière que forme la chaîne du Say Phu Pha Nang, surtout sur son flanc occidental. Le site se trouve en effet sur la seule route qui permette de descendre (par une passe) dans la vallée de la Nam Thon, à une quinzaine de kilomètres au sud-ouest - outre, naturellement, la voie qui longe le Mékong, bien plus au sud, et celle qui se trouve à l'extrémité du Say Phu Pha Daeng, bien plus au nord. Il y a donc bien une logique géographique dans le choix de cette zone.

Les sema môns de Ban Nong Khon sont dressés tout autour du temple moderne du village. On en compte une douzaine, mais neuf parmi eux ont vraiment fait l'objet d'un travail soigné, et seuls six portent l'image du stūpa (un seul exemple pour une sculpture sur les deux faces). Parmi ceux-ci, deux ont, autour de cette image (traitée en forme d'épine, voire de «tige »), des motifs qui lui sont liés à la base et qui s'en écartent en s'élevant, comme des feuilles. On reconnaîtra une facture semblable autour de Vientiane. Un des sema non historiés montre quatre côtés plus ou moins égaux en largeur (entre 30 et $35 \mathrm{~cm}$ ) et une hauteur hors du sol de $96 \mathrm{~cm}$, soit une taille bien supérieure aux autres vestiges. Le temple conserve une statue de Buddha méditant de facture assez maladroite, mais la couche d'enduit et de peinture qui la recouvre cache peut-être une production ancienne.

\section{Le bassin de la Nam Hum}

La Nam Hum est un petit affluent non navigable de la Nam Ngum qui prend, comme la Nam Cheng, sa source sur le massif du Say Phu Pha Nang. Le confluent se trouve à Tha Ngon, là où la Nam Ngum qui suivait jusque-là une direction sud opère une grande boucle pour se diriger résolument vers l'est, où elle finit par rejoindre le Mékong. Les rives mêmes de la Nam Hum n'ont pour le moment révélé aucun vestige, mais son bassin, auquel appartiennent d'autres petits cours d'eau comme le Huay Sone et la Nam Khone, montre un ensemble cohérent de plusieurs sites que dominait certainement le sanctuaire de Dan Sung, placé sur un plateau, à quelque $20 \mathrm{~km}$ à peine à l'ouest du cours de la Nam Ngum. 


\section{Dan Sung}

Dan Sung, comme son nom l'indique (« domaine haut »), est un site surélevé. D'une altitude moyenne de $290 \mathrm{~m}$, il apparaît comme le contrefort avancé de la chaîne de montagnes qui enferme à l'ouest toute la plaine de Vientiane. Cette vaste plate-forme, qui présente une falaise sur son flanc oriental, a dû exercer un fort attrait sur les populations anciennes en raison de son caractère physique particulier et saisissant. Il s'agit en effet d'un amoncellement d'énormes blocs de grès où il est possible de se faufiler avec plus ou moins de facilité. Le lieu comporte de véritables grottes auxquelles on accède par des boyaux, mais il apparaît d'abord comme un ensemble d'abris-sous-roche. S'il n'est pas possible, à ce jour, de prouver que Dan Sung a été un lieu d'habitat pour une grosse communauté, il est sûr en tout cas, au vu des témoignages significatifs dont on dispose, que le site a eu une grande importance sur le plan religieux.

S. Karpelès et P.-M. Gagneux ont en effet décrit avec quelques détails Dan Sung qu'ils ont reconnu comme un important site religieux pré-lao. Le second l'identifie clairement à un site môn - alors que la première évoque à propos de ses vestiges l'art de Lavapuri (Lopburi), qui est généralement présenté comme une synthèse de l'art khmer angkorien et de l'art môn de Dvāravatī. Tous deux omettent toutefois certains vestiges qui permettront peut-être de préciser les affiliations.

L'cnscmblc des témoignages archéologiques est pour l'instant réparti dans deux endroits distincts qui constituent en quelque sorte les extrémités nord et sud du site. La distance qui les sépare n'est que de $150 \mathrm{~m}$ à vol d'oiseau, mais le « dédale de rochers et de cavernes fort pittoresques " (dit S. Karpelès) qu'on est obligé d'emprunter pour relier les deux points fait souvent croire à une distance plus longue.

La première « grotte » $\left(\mathrm{n}^{\circ} 12\right.$, selon une nomenclature déjà utilisée dans les années 1970) qu'on rencontre en arrivant du sud, par l'unique chemin d'accès au plateau, est occupée par plusieurs images. Cependant, comme le soulignait déjà $\mathrm{S}$. Karpelès, « la seule statue qui vaille la peine d'être mentionnée [les autres sont des productions relativement récentes, de petite taille et en ronde-bosse] est celle d'un Buddha méditant, sculpté à même le roc, de $1 \mathrm{~m}$ de haut et $90 \mathrm{~cm}$ de large. De chaque côté, se trouvent des protubérances que la population désigne sous le nom de têtes d'éléphants ; mais l'épaisse couche d'enduit qui les recouvre ne permet de faire aucune conjecture à leur sujet ${ }^{26}$ " (fig. 19). Nous en oserons tout de même une : par leur forme, ces deux protubérances rappellent fort les deux motifs couplés et bien mis en relief qui se trouvent de part et d'autre du plus grand Buddha de Vang Sang - que S. Karpelès elle-même identifiait à des fleurs de lotus. On verra plus bas que la correspondance existant entre les deux sites sur le plan de l'iconographie ne se limite pas à ces deux seules représentations de motifs « décoratifs ", et il fait peu de doute que les sculpteurs ont voulu représenter ici le même type d'insigne. Les remarques de $\mathrm{S}$. Karpelès sur l'enduit qui recouvre les protubérances valent également pour le Buddha lui-même, dont le vêtement et l'écharpe sont très certainement un ajout fait à une époque plus récente.

La seconde " grotte », à laquelle est donnée depuis les années 1970 (au plus tard) le numéro 11 , est comme la première un abri-sous-roche, constitué d'une base assez étroite que recouvre un énorme chapeau en pierre (fig. 20). Ce type de composition géologique se retrouve ailleurs sur le site, parfois de façon plus imposante encore - et peut être

26. S. Karpelès, « Les grottes sculptées... », op. cit., p. 771. 
également observé dans d'autres lieux qui ont été utilisés de la même façon, comme sur le site de Phu Phra Bat dans la province thaïlandaise d'Udon Thani ${ }^{27}$.

Le choix de cet abri-sous-roche pour y établir un sanctuaire est bien sûr lié à sa configuration « accueillante » et facilement exploitable, mais il tient sans doute aussi à sa position tout en bordure - puisque ses côtés immédiats nord et est se situent déjà dans la pente qui dévale rapidement vers la plaine.

L'intérêt porté à cet endroit s'était jusqu'à présent limité à un ensemble d'images sculptées en bas-relief sur les flancs ouest et sud du bloc rocheux, qui a une forme globalement ovale (fig. 21). De gauche à droite, en leur faisant face, on observe d'abord dans un premier pan de paroi, sculptés à l'intérieur d'une niche, deux Buddha assis d'une hauteur de 1,70 m environ, en position de méditation. Leur apparence initiale a été modifiée par des enduits, en particulier pour le premier dont la partie inférieure et la coiffe ont certainement été refaites. Son visage semble cependant conserver des traits originaux très marqués. Le second a un aspect plus grossier, notamment le visage émacié, mais ses jambes paraissent avoir gardé leur disposition originale qui est celle qu'on retrouve également sur la plupart des images de Vang Sang (vajrāsana). À droite de ce Buddha, on pouvait encore voir dans la première moitié du $\mathrm{Xx}^{\mathrm{C}}$ siècle une image de Phra Kaccay qui a disparu ensuite. Cette image était à l'évidence une production tardive, en stuc, collée à la paroi.

Le premier pan de paroi fait alors place à une anfractuosité permettant d'accéder au cœur de la base sur laquelle repose l'énorme roche qui forme un couvercle. C'est à cet endroit qu'on peut trouver, dans un bloc de grès indépendant d'environ $1,50 \mathrm{~m}$ de haut, un groupe de trois Buddha debout, placés dos à dos - dont deux ont une main levée (la droite pour l'un, la gauche pour l'autre), les autres mains étant placées le long de leur corps. Ces images ont été largement recouvertes par des enduits, et les quelques traits originaux qu'on peut encore relever concernent le plissé des vêtements dans leur partie inférieure (sous la ceinture), ainsi que la présence, sur l'une des statues, de deux bracelets (S. Karpelès n'en a vu qu'un; aussi l'autre est-il peut-être un ajout postérieur). À droite de ce groupe, indépendant lui aussi de la roche, figure un "Bouddha debout, hauteur $1 \mathrm{~m}$. 50, largeur des épaules $0 \mathrm{~m} .63$, dont la tête, le cou, la main droite et le pied gauche sont de grossières réparations de date toute récente ; la main gauche pend le long du corps, la paume à l'intérieur, et le vêtement couvrant le buste jusqu'aux poignets se compose d'une étoffe dont le jabot est retenu par une ceinture en relier, qui descend jusqu'aux chevilles et forme sur le devant un pli creux à double pan relevé de chaque côté ; le bras droit et l'avant-bras droit, dont la main est relevée, la paume à l'extérieur, sont ornés de bracelets en relief, et un collier en forme de pétales dentelés, se détache également en relief autour du $\operatorname{cou}^{28}$ ». On peut compléter cette description en remarquant qu'il y a peu de chances pour que le corps ait à l'origine été prolongé par une telle tête. La taille énorme et totalement disproportionnée de cette dernière ne peut s'expliquer que par l'ajout d'enduits (sauf pour la coiffure), d'autant que certains traits bien marqués du visage ont subsisté. Il s'agit certainement d'une tête de substitution qui provenait d'une autre image. Les bijoux et la ceinture mis à part, le corps nous rappelle beaucoup celui qui est aujourd'hui conservé, sans tête, dans la galerie occidentale du That Luang sous le $\mathrm{n}^{\circ} \mathrm{TL} \mathrm{I} / 24$. Le drapé est quasiment identique, et les deux statues possèdent une face

27. Ce site fait d'ailleurs partie du même massif s'étirant sur les dcux rives du Mékong, dans unc direction générale nord-sud. Il n'est distant de Dan Sung que d'une quarantaine de kilomètres à vol d'oiseau.

28. S. Karpelès, ibid. 
postérieure plate (il s'agit donc d'une fausse ronde-bosse), comme si elles étaient en fait adossées à une stèle qui s'arrête au niveau de leurs épaules. Si le collier et la ceinture figurent bien parmi les motifs ornementaux d'origine, ils pourraient nous rappeler, par leur traitement, des exemples qu'on trouve dans la sculpture khmère. La dernière grande image de la grotte est un Buddha assis en haut-relief d'une hauteur de 1,70 m environ, adossé à une stèle. S. Karpelès rapporte à son propos que seuls les avant-bras et la tête sont originaux. Ce n'est plus l'impression que donnent les premiers aujourd'hui, mais la seconde conserve effectivement des traits très marqués, dont la présence d'une moustache n'est pas le moindre.

À ces statues remarquées par tous les visiteurs il faut ajouter quelques vestiges qui ne sont pas toujours mentionnés. À droite du Buddha « moustachu », ou plutôt derrière lui, sculptée sur la paroi orientale du bloc rocheux, se trouve l'ébauche d'un Buddha assis que le regard n'identifie pas forcément tout de suite. Le modelé général du corps reste reconnaissable, mais la tête a pratiquement disparu. La partie inférieure d'un Buddha assis en ronde-bosse, très abîmée (jambes, avant-bras), est par ailleurs posée sur un rebord de roche, à l'endroit où se trouvait au début du $x^{\mathrm{e}}$ siècle le Phra Kaccay en stuc. Une photographie ancienne (fig. 22) montre qu'il était associé à deux autres fragments en grès appartenant à des images très distinctes : une base avec des pieds (il en existe également une dans la galerie orientale du That Luang, mais elle est différente) peutêtre à mettre en rapport avec le Buddha debout -, et le côté droit de la tête d'un Buddha sculpté en bas-relief qui semble avoir été arraché de la roche. Il s'agit probablement de la pièce issue de la grotte 11 que releva P.-M. Gagneux dans son inventaire et qu'il transporta lui-même, en 1969, au Vat Ho Phra Kèo où elle reçut d'abord le $n^{\circ} 1198 / 1^{29}$. Avec son modelé subtil, ce fragment de visage non modifié par les ajouts d'enduits est l'une des pièces qui montrent le mieux l'influence de l'art khmer. Un autre vestige peut être associé à cet ensemble : il s'agit d'un bloc de pierre de $34 \mathrm{~cm}$ de hauteur qui, par sa face antérieure, sculptée, pourrait correspondre à un petit sema (base resserrée sur laquelle apparaissent trois bandes superposées, dont la plus haute semble figurer trois feuilles ; partie supérieure en forme d'accolade) - mais ses côtés et sa partie antérieure étant demeurés totalement bruts, il s'agit manifestement d'un objet inachevé.

Nous avons évoqué la présence d'une ébauche de Buddha sur la paroi orientale de la grotte 11 de Dan Sung. Cette partie à ciel ouvert présente bien d'autres traits intéressants. Il apparaît d'abord certain qu'elle possédait autrefois, tout comme les autres faces du sanctuaire sous-roche, une épaisse couverture sur toute sa longueur (environ $7 \mathrm{~m}$ ). Celleci a été brisée et repose au sol à $2 \mathrm{~m}$ environ de l'ensemble, à la suite d'un glissement. La rupture paraît bien avoir précédé les différents travaux de sculpture, mais ceux-ci ont pu également la provoquer. En chutant, l'énorme bloc de grès qui a longtemps constitué une sorte de plafond a laissé subsister, au niveau de la base rocheuse, un rebord plat, perpendiculaire à la paroi verticale, qui constitue une sorte de chemin, large d' 1 m environ. Il apparaît que ce rebord a été exploité à des fins utiles, puisqu'au moins cinq cavités aux formes plutôt régulières y ont été creusées assez profondément (fig. 23). L'extrémité nord se caractérise en particulier par la présence de deux mortaises rectangulaires dépassant $40 \mathrm{~cm}$ de longueur - et, pour l'une, $30 \mathrm{~cm}$ de largeur. Au centre, deux autres mortaises circulaires ont un rayon respectif de $25 \mathrm{~cm}$ et $14 \mathrm{~cm}$. Une cinquième ouverture consiste en un trou circulaire creusé dans un cadre carré. La fonction de ces cavités ne donne lieu qu'à des conjectures : emplacements destinés à recevoir les tenons de statues

29. P.-M. Gagneux, Les sites anciens..., op. cit., pièce Ml1. Elle n'a pas encore été retrouvée. 
ou des piliers, cuves, dépôts? Un autre creux de forme circulaire occupe le centre de la « grotte » même, c'est-à-dire dans la partie couverte. Si ce type d'aménagement au sol s'avère pour l'instant unique au Laos, on ne peut s'empêcher d'opérer un rapprochement avec les mortaises rectangulaires creusées dans la paroi verticale de Vang Sang - quoique leurs fonctions étaient sans doute très différentes.

Un autre aspect fondamental - jusqu'ici passé sous silence - de la richesse archéologique de Dan Sung est la présence, autour de la grotte 11, de plusieurs sema. Six de ces pièces ont pour l'instant été identifiées, mais il n'est pas interdit de penser, vu la difficulté d'accès à certaines parties du terrain (végétation dense et sol en pente), que d'autres pourront encore être découvertes. Elles se présentent toutes comme des stèles à deux faces, très bien taillées, avec un sommet en forme d'accolade (l'usure a arrondi la pointe sur certaines pièces). Ces sema ont d'importantes dimensions, puisqu'ils atteignent tous une hauteur minimale de 1,50 m (l'un d'eux atteint $1,90 \mathrm{~m}$ ), une largeur de $70 \mathrm{~cm}$ au moins, et une épaisseur supérieure à $30 \mathrm{~cm}$. On peut sans conteste les considérer comme le groupe le plus important, en termes de volume, de toute la plaine de Vientiane. Aucun d'entre eux n'est historié (l'image du stüpa en est totalement absente), mais l'une de ces stèles (côté nord-est), qui se distingue également par sa forte base, plus épaisse et bien marquée, porte sept lignes d'écritures (fig. 24). Le texte est très effacé et il n'a pas encore été possible de produire une transcription complète de cette inscription, préalable à une traduction. Quelques observations peuvent toutefois être faites sur la composition d'ensemble qui présente des caractéristiques intéressantes. Les trois premières lignes semblent être en caractères " post-pallava », alors que les quatre autres consistent en des caractères khmers de l'époque angkorienne - certaines consonnes étant bien visibles avec les « cheveux » qui ornent leur partie supérieure ${ }^{30}$. Chaque ligne révèle en son centre une césure, et de part et d'autre sont apparemment réparties deux séries de huit syllabes (les consonnes, simples ou doublées, sont identifiables, de même que les voyelles suscrites et souscrites). Il y a donc tout lieu de penser que le texte est versifié et que sa langue est le sanskrit ${ }^{31}$. On aurait alors ici un témoignage d'écrit religieux tout à fait exceptionnel pour la plaine de Vientiane - l'exemple de la stèle de Say Fong étant mis à part - mais qui n'est pas absolument surprenant, puisque des inscriptions sanskrites liées aux contextes môn et khmer ont été retrouvées sur la rive droite du Mékong, dans le nord-est de la Thaïlande ${ }^{32}$. En attendant une confirmation ou une infirmation de cette hypothèse, on peut déjà mettre en évidence des points qui nécessitent une réflexion. Comment faut-il interpréter le fait, en premier lieu, que l'inscription soit manifestement rédigée avec deux écritures différentes, appartenant en principe à des périodes bien démarquées ? Est-il possible de croire à des phases de rédaction appartenant à des contextes culturels distincts, puisque la marque de l'écriture khmère s'oppose bien à l'identité supposée mône de la grande majorité des vestiges rencontrés jusqu'à présent dans la plaine de Vientiane ? Les sites rupestres de Vang Sang et de Dan Sung, à l'instar de certains sites de la même catégorie situés à proximité sur la rive droite du Mékong (Phu Phra Bat étant le meilleur exemple), ont certainement été occupés pendant plusieurs siècles, de façon

30. Cette impression est confirmée par D. Soutif qui ajoute - avec prudence, car les images communiquées étaient de mauvaise qualité - que les caractères pourraient dater des $1 \mathrm{X}^{\mathrm{e}}-\mathrm{X}^{\mathrm{e}}$ siècles.

31. La présence du terme $\dot{s} r \bar{\imath}$ est certaine, de même que des visarga en finale. $\mathrm{Ch}$. Bauer précise que cela existe également dans des inscriptions en langue mône. Des mots spécifiquement môns n'ont cependant pu être identifiés pour l'instant (communication personnelle).

32. Ch. Bauer, "Notes on Mon Epigraphy ", Journal of the Siam Society 79/1, 1991, p. 31-83, en particulier le tableau $\mathrm{H}$, p. 56 . 
continue, par des populations qui se sont mélangées et dont les pratiques ont évolué. C'est peut-être le point de vue de $\mathrm{S}$. Karpelès lorsqu'elle rapporte, à propos des deux sites qu'elle a visités: "Si l'on examine les traits des visages des diverses statues qui se trouvent dans ces grottes, on est frappé par la similitude qui existe entre leur facture et celle qui caractérise l'art khmèr (arcades sourcilières horizontales, manière de traiter le nez et la bouche, etc.), alors que les représentations de boucles de cheveux, la façon d'indiquer le vêtement ainsi que les diverses attitudes, portent encore l'empreinte de l'art de Dvāravat $\overline{1}^{33}$ ». Elle conclut alors à un art de synthèse, comme celui de Lavapuri (Lopburi) «qui a rayonné jusque dans des régions excentriques, du $x^{\mathrm{e}}$ siècle aux $\mathrm{xl}^{\mathrm{e}}$ et $\mathrm{XII}^{\mathrm{e}}$ siècles ». Dans le cas de Dan Sung, les vestiges identifiés pourraient peut-être se rattacher aux deux derniers siècles du premier millénaire.

\section{Ban Na Sone}

Le village de Ban $\mathrm{Na}$ Sone est situé à $7 \mathrm{~km}$ au sud-est de Dan Sung et à $5 \mathrm{~km}$ environ de la pente qui y mène. Il tend aujourd'hui à se confondre avec Ban Hua Khua, établi sur la route $n^{\circ} 13$, à l'endroit où celle-ci passe au-dessus du Huay Sone, ruisseau dont la source est très proche de Dan Sung, sur son flanc septentrional. Depuis une date indéterminée, le temple de Ban Na Sone abrite, plantés sur le côté est du sanctuaire, deux sema de taille moyenne (parties visibles inférieures à $60 \mathrm{~cm}$ de hauteur) qui portent tous les deux l'image du stüpa. Pour l'une des pièces, pratiquement collée au mur, cette image n'est plus identifiable que sur une face : elle est alors très stylisée, réduite à une pure forme en épine. La seconde pièce révèle l'image sur ses deux faces, mais traitée de façon différente : un côté montre un stüpa élancé, marqué de deux doubles bandes horizontales entourant une partie centrale légèrement arrondie (le kumbha?) ; l'autre côté présente une image un peu plus élaborée, avec un sommet formé de motifs en flammèches, qu'on retrouve sur un sema proche, dont nous parlerons bientôt. Cette correspondance est peutêtre significative quant au véritable lieu de provenance de ces deux vestiges.

À $800 \mathrm{~m}$ au nord-ouest du temple de Ban Na Sone ont été mis au jour, à l'occasion de l'aménagement d'un terrain de football, sur deux sites distants l'un de l'autre d'une centaine de mètres à peine, plusieurs vestiges môns qui ont contribué à renforcer l'intérêt pour cette zone proche du Huay Sone. Le premier site a été découvert en mars 2005 : il a révélé cinq grands blocs de pierre sculptés pouvant être assimilés à des sema, plus quelques autres fragments, dont deux au moins appartiennent également à des bornes bouddhiques. La forme très suggestive d'un des grands vestiges nous avait conduit à le confondre, dans un article précédent, avec un linga. Une étude plus attentive de ce genre de sculptures nous fait conclure aujourd'hui de façon quasi certaine que l'extrémité de la pierre, sculptée à l'image d'un gland, se trouve être en fait la partie inférieure - donc la base - d'un sema non historié (fig. 25) ${ }^{34}$. Il est intéressant de constater que les quatre autres grands sema ne se ressemblent pas - et que chacun d'eux semble avoir été sculpté dans une roche différente, les couleurs variant du rose très prononcé au blanc, en passant par le gris. La plus grande de ces pièces mesure 1,80 m et est marquée d'une fine double

\section{S. Karpelès, « Les grottes sculptées... », op. cit., p. 771-772.}

34. M. Lorrillard, «Pour une géographie historique... », op. cit., p. 124. L'identification avec un linga était renforcée par l'interprétation que les habitants lao donnèrent immédiatement de cette pièce, et qui les conduisit à organiser très rapidement des rituels liés à la fertilité, avec utilisation par les jeunes femmes d'une eau consacrée par l'objet pour des ablutions. La même erreur a été faite avec d'autres vestiges à l'aspect semblable répartis ailleurs dans la plaine de Vientiane (Ban Thin Kèo, Ban Muang Kao, Ban Sapheu). 
bande horizontale qui sépare la base normalement enterrée de la partie destinée à rester visible. La forme ogivale de cette dernière est très irrégulière et fait pencher l'ensemble vers un côté. À cette facture assez fruste s'oppose celle d'un sema à deux faces plates, un peu moins grand, mais dont la base est plus large que la partie supérieure. Une frise que constituent deux fines bandes enfermant des motifs ronds en forme de boutons, lesquels alternent avec des espèces de trèfles à trois feuilles, assure ici la transition. Le sommet de cette stèle en forme d'ogive a été brisé. La partie qui s'en était détachée est maintenant (2011) fixée de façon précaire par un ruban. À la hauteur de cette brisure figurent les restes à peine discernables d'au moins trois rangées (pour ne pas dire lignes) de caractères. Parmi eux, deux sont plus visibles et sont ornés à leur sommet des « cheveux » qui caractérisent l'écriture khmère déjà développée. La forme des lettres, notamment celle d'un « $\mathrm{k}$ » et d'un « $\mathrm{t}$ », paraît cependant encore bien archaïque. Étant donné l'état de l'inscription, il est fort peu probable que son objet soit jamais identifié ${ }^{35}$. Un quatrième sema, dont la base et la partie supérieure sont manquantes (elles figurent peut-être parmi les fragments découverts à proximité), ne semble pas avoir été véritablement achevé par le sculpteur, à moins qu'il n'ait davantage subi l'usure du temps que les autres pièces. Il est encore possible de discerner une partie du bandeau supérieur de la base, ainsi que certains motifs qu'elle coiffait. Leur dessin est très proche de celui que nous avons associé à un trèfle à trois feuilles. Le cinquième sema est moins bien conservé, puisque tout un côté est manquant ou fortement abîmé. L'autre côté montre la forme générale (ogive), ainsi qu'un traitement sur deux plans, la partie centrale étant proéminente, comme si elle était encadrée.

Sur le même terrain de Ban Na Sone, quelques mois plus tard, ont été mis au jour de nouveaux vestiges en deux points distants l'un de l'autre d'une vingtaine de mètres, mais appartenant probablement à un même site, distinct de celui que nous venons d'évoquer. Le point le plus au nord a révélé deux sema dont l'un, plus grand, est pratiquement couché sur l'autre et le recouvre en grande partie. La facture de la pièce principale est exceptionnelle. Elle est légèrement inférieure en taille aux grands sema de Dan Sung, mais elle s'en distingue surtout par sa forme très élaborée, en ogive avec côtés biseautés, et par sa large base ornée d'une frise avec pétales de lotus (fig. 26). La face visible est très plate et peut laisser croire qu'elle était destinée à porter une inscription. Le retournement de la stèle donnera peut-être lieu à une agréable surprise. Le second sema, couvert, ressemble au premicr, mais il faudra attendre son dégagement complet pour pouvoir établir une véritable comparaison. Le point le plus au sud a révélé deux autres sema accolés, mais de taille inférieure et historiés. Ce sont en effet les seuls vestiges de ce terrain de Ban $\mathrm{Na}$ Sone qui portent l'image du stūpa. Le premier, en grès rose, montre un traitement tout à fait particulier, puisqu'au-dessus de la base du stūpa, arrondie et figurant manifestement le kumbha, se trouvent d'abord une espèce de niche qui ne renferme rien - hormis deux formes rondes dans les parties inférieure et supérieure (la plus haute pourrait être la tête d'un petit Buddha dont le corps a disparu) -, puis une flèche composée d'éléments qui font penser à des flammes (fig. 27). Cette dernière partie rappelle le motif supérieur du stūpa de l'un des deux sema conservés au temple de Ban $\mathrm{Na}$ Sone. Le deuxième sema, sculpté dans un grès beaucoup plus clair, est brisé en plusieurs morceaux. Toutefois la partie inférieure montre clairement un stüpa en forme d'épine reposant sur une large

35. Ch. Bauer, qui a vu récemment l'épigraphe, ne peut émettre une conclusion sur la langue utilisée, car il est impossible de restituer un mot complet (communication personnelle, 2011). 
bande horizontale. Au centre de cette dernière a été sculpté un cartouche rectangulaire qui comporte apparemment une fleur avec un bouton entouré de quatre pétales.

\section{Ban Nong Khan Khu}

Le village de Ban Nong Khan Khu borde la route $\mathrm{n}^{\circ} 13$ et n'est établi qu'à $1 \mathrm{~km}$ au nord de Ban Na Sone. Son temple conserve apparemment, depuis une trentaine d'années, quatre vestiges en grès qui ont été mis au jour dans une zone de rizières située à quelques centaines de mètres à l'ouest. Le lieu de découverte se trouve donc à environ $1300 \mathrm{~m}$ au nord-est des sites précédemment mentionnés. Ces quatre vestiges sont très distincts. Il s'agit d'abord de deux sema ornés chacun d'un stüpa sur leurs deux faces. Le premier, dont il ne subsiste que la partie inférieure, présente pour chaque fragment d'image une base très large, divisée en plusieurs sections. Il est probable que la partie supérieure des stüpa était d'un aspect beaucoup plus effilé. Le second sema est complet et montre des stüpa en forme d'épine. À ces stèles semblaient être associés une grosse tête de Buddha haute d'une quarantaine de centimètres et un Buddha assis en position de méditation. La tête manque à ce corps d'une hauteur de $54 \mathrm{~cm}$. Il ne peut s'agir de la pièce précédente qui appartenait à une image beaucoup plus grande. La facture de ces deux vestiges correspond à celle des images que nous avons identifiées comme étant typiquement mônes.

\section{Ban Ilai}

Après Ban Nong Khan Khu, la route $\mathrm{n}^{\circ} 13$ traverse, en direction du nord, le village de Ban Ilai où sont disposés, autour d'un petit tertre - apparemment les restes en briques d'un sanctuaire lao -, neuf sema. Leur position respecte celle des points cardinaux et subcardinaux, sauf sur le côté nord qui compte un sema supplémentaire. Il y a peu de chances pour que cette disposition soit celle qui avait été prévue à l'origine. Le temple moderne du village est situé à $200 \mathrm{~m}$ au sud et présente lui-même, coulé dans le parvis en ciment qui l'entoure, le sommet ogival d'un autre sema appartenant probablement au même groupe. Parmi ces stèles de taille assez imposante (la plus grande laisse voir une hauteur hors du sol de $1,50 \mathrm{~m}$, la plus large dépasse les $90 \mathrm{~cm}$ ) et de facture soignée - elles possèdent chacune deux faces plates et un sommet de forme ogivale ou arrondie -, trois seulement portent l'image stylisée du stüpa en forme d'épine. Celle-ci n'est cependant parfaitement visible que sur un seul sema, sis dans l'angle nord-est. Le site de Ban Ilai est situé à $7 \mathrm{~km}$ au nord-est de Dan Sung, c'est-à-dire à la même distance que celui de Ban Na Sone, au sud-est.

Extérieur au triangle que forment ces trois points, car situé à $5 \mathrm{~km}$ au nord de Ban Ilai, se trouve le village de Ban Na Nat. À proximité de celui-ci, P.-M. Gagneux avait remarqué en 1977, sur le sommet d'un tertre d'où émergeaient des briques, une tête de Buddha en grès de facture ancienne. Ce vestige n'a pas été retrouvé (le tertre n'a pas encore été identifié non plus), mais il peut figurer parmi les pièces qui ont été rapportées ensuite au Vat Ho Phra Kèo et au That Luang pour y être conservées. Ban Na Nat n'est situé qu'à quelques centaines de mètres du cours de la Nam Hum. Il constitue peut-être la limite septentrionale de ce groupe de sites que nous avons placés dans le bassin de cette rivière.

\section{La rive gauche de la Nam Ngum et le bassin de la Nam Kham}

À une latitude intermédiaire entre celles des bassins de la Nam Cheng et de la Nam Hum se trouvent deux sites établis à proximité des ruisseaux qui naissent sur le massif du Phu Khao Khuay et qui se rejoignent rapidement pour grossir le cours de la Nam Kham, affluent de la Nam Ngum sur sa rive gauche. Par leur emplacement, ces sites font donc 
pour l'instant exception, mais toute cette région de la plaine de Vientiane acculée au nord par une chaîne de montagnes est encore très peu développée, et des aménagements futurs permettront peut-être de faire de nouvelles découvertes.

\section{Ban Sapheu}

Le village de Ban Sapheu est situé à $1 \mathrm{~km}$ au nord du Huay Thon - petit ruisseau qui, lorsqu'il atteint la plaine, suit une direction sud-ouest pour rejoindre la Nam Kham, puis la Nam Ngum. Le temple de ce village ne conserve plus qu'un seul témoignage véritablement ancien, mais celui-ci est tout à fait exceptionnel par sa facture. Il s'agit d'un sema octogonal, d'une hauteur visible de 1,80 m (la base enchâssée dans le sol ne doit pas mesurer moins de $40 \mathrm{~cm}$ ) et d'une circonférence de $1,50 \mathrm{~m}$, avec un sommet demi-sphérique. La partie inférieure encore visible montre deux bandes décoratives horizontales attachées, l'une constituée d'une rangée de boutons circulaires, l'autre consistant en une frise de motifs en forme de feuilles. Ce vestige n'était sans doute pas unique, puisque la galerie occidentale du That Luang conserve depuis 1976 un sema de facture totalement analogue - y compris pour les bandes décoratives - qui est référencé comme étant originaire de Ban Sapheu (fig. 28). Couché sur le sol, sa longueur totale est de $2,22 \mathrm{~m}$, mais elle est réduite à $1,81 \mathrm{~m}$ si elle s'arrête à la limite où commence la base, après les deux frises. Les deux sema semblent donc avoir été de hauteur égale, même si le second présente une circonférence un peu plus importante $(1,75 \mathrm{~m})$. Deux sema de cette taille - dont on ne peut trouver des équivalents au Laos qu'à Ban Kang, dans la province de Savannakhet - ne pouvaient être associés qu'à un site de grande importance.

\section{Ban Na Pheng}

À $2,6 \mathrm{~km}$ au nord du temple de Ban Sapheu, à proximité immédiate du village de Ban Na Pheng, entre deux ruisseaux (le Huay Hong Pheng à $250 \mathrm{~m}$ au nord et le Huay Kut à $450 \mathrm{~m}$ au sud) qui se rejoignent un peu plus loin pour aller gonfler au sud-ouest les cours de la Nam Thon et de la Nam Kham, le jardin d'un particulier donne à voir six blocs de grès en partie enterrés qui, malgré leur forme assez grossière, sont le produit d'un façonnage par l'homme et peuvent certainement être considérés comme des sema. Aucun n'est historié. Sur le même lieu, semble-t-il, ont été retrouvées dans les années 1970 deux autres pièces qui sont conservées dans la galerie occidentale du That Luang. La première, enregistrée sous le $n^{\circ}$ TL I/19, cst un sema de forme presque ovale qui présente sur l'une de ses faces l'image d'un stüpa très trapu et divisé en plusieurs sections. La facture reste assez grossière. Il est possible que le second sema enregistré comme provenant de Ban Na Pheng soit celui qui porte le $n^{\circ}$ TL I/41 (et non TL I/15 ainsi que semble l'indiquer l'inventaire), car celui-ci montre un aspect assez semblable, bien qu'un peu plus effilé, tant pour ce qui regarde la forme du matériau que celle du stüpa. Les vestiges de Ban Na Pheng doivent être associés à ceux de Ban Sapheu, vu leur proximité. Il est cependant difficile de les confondre en un même groupe, tant la maîtrise des sculpteurs s'avère différente.

\section{Les bords du marais du That Luang}

De tous les sites supposés môns identifiés dans la plaine de Vientiane, ceux qui se trouvent dans la vicinité du That Luang forment peut-être le groupe le plus surprenant, et par là même celui qui suscite le plus de questions. La proximité de Vientiane peut tout d'abord porter à croire que ces emplacements, où des vestiges caractéristiques ont été retrouvés, étaient intimement liés à la berge où la ville lao ancienne a été établie. Cet 
espace aurait alors constitué une sorte de centre d'où auraient rayonné vers la plaine des zones d'occupation moins importantes. Une attention plus précise portée à la configuration du terrain montre toutefois que cette impression est très probablement biaisée, car les sites identifiés se démarquent nettement de la rive du Mékong. Les vestiges découverts in situ les plus rapprochés du fleuve sont en effet positionnés sur le versant oriental de la colline du That Luang (donc à l'opposé de celui qui descend vers la ville), et les autres s'inscrivent pleinement à l'intérieur des terres, soit vers l'est, soit vers le sud, jusqu'à une certaine limite. Le dénominateur commun de tous ces sites est en fait le marais - ou l'étang (beung) - du That Luang, autour duquel ils sont positionnés, sur des bordures légèrement élevées. Une photographie datée de 1946 montre que ces zones exondées étaient caractérisées par une couverture végétale importante, qui a aujourd'hui pratiquement disparu (fig. 29). Le marais du That Luang constitue une étendue très longue (près de $10 \mathrm{~km}$ ) et assez étroite (il n'excède pas 2,4 km de largeur), nettement orientée nord-sud. Il est loin d'être isolé de la Nam Ngum, dont la boucle la plus proche est à $18 \mathrm{~km}$, puisque des études ont montré que des cours d'eau et des canaux mettaient en relation cette rivière avec le Mékong au niveau de Vientiane - en passant par de larges réservoirs, comme l'étang du That Luang, lesquels servaient à réguler les crues importantes de l'un ou l'autre des deux cours d'eau $^{36}$. Si les vestiges retrouvés relèvent tous de pratiques rituelles et furent donc attachés à des sanctuaires, le cadre physique auquel ils sont liés montre toutefois que la présence permanente de l'élément liquide fut la raison principale du choix des sites - et que ceux-ci étaient avant tout des lieux où l'activité humaine était intense ${ }^{37}$.

\section{Vat Ban Phon Pa Nao}

Le point le plus septentrional de cette ceinture entourant le marais du That Luang - et qui constitue en quelque sorte son "sommet»-est l'espace occupé par le domaine du temple de Ban Phon $\mathrm{Pa} \mathrm{Nao}$, "village » aujourd'hui entièrement intégré à Vientiane, à $1700 \mathrm{~m}$ au nord-est du That Luang. Le nom du temple indique une éminence (phon) qui est peut-être moins due à l'altitude du terrain elle-même - à peine supérieure à celle des rizières qui l'entourent - qu'à la présence passée d'un tertre qui ne serait plus visible aujourd'hui. Selon P.-M. Gagneux, le monastère a été reconstruit en 1959 (il devait alors être totalement en ruine) et l'on a mis au jour à cette occasion deux pièces anciennes en grès qui ont été référencées dans l'inventaire de 1977 : un Buddha assis en méditation adossé à une stèle en forme de nimbe (M30), et une stèle sur laquelle apparaissent au centre, en bas-relief, deux personnages, dont le Buddha assis (M76) ${ }^{38}$. Ces deux pièces existent toujours et n'ont pas quitté le site. La première, haute de $79 \mathrm{~cm}$, n'est pas forcément reconnaissable sur l'instant, car elle a été encastrée dans l'autel du sanctuaire et recouverte d'une bonne couche de peinture dorée. Il serait aisé, aujourd'hui, d'y voir une production contemporaine. La seconde est beaucoup plus visible, puisqu'on a bâti à son intention un petit pavillon qui la protège des intempéries, tout en gardant ses

36. Christian Taillard, « Les berges de la Nam Ngum et du Mékong : systèmes économiques villageois et organisation de l'espace dans la plaine de Vientiane (Laos) », Etudes rurales 53-56, Agriculture et sociétés en Asie du Sud-Est, p. 119-168; «Devenir de l'ancien système hydraulique de Vientiane : la place du patrimoine technique et des héritages paysagers dans le développement urbain ", dans Recherches nouvelles sur le Laos, op. cit., p. 339-359.

37. Les observations qui suivent datent de 2011. Depuis, le «Beung That Luang » a été modifié par des aménagements importants qu'ont financés de grands groupes étrangers. Si des vestiges archéologiques ont été découverts à l'occasion de ces travaux, ils n'ont pas été révélés et conservés dans les lieux adéquats.

38. P.-M. Gagneux, Les sites anciens..., op. cit. 
quatre côtés ouverts (fig. 30). P.-M. Gagneux donnait à cette stèle une hauteur de 1,97 m (la partie inférieure est aujourd'hui insérée dans la maçonnerie qui lui sert de socle) et voyait " sur l'une de ses faces un Bouddha assis en méditation à la gauche duquel se tient un personnage debout ". Si la remarque concernant le Buddha ne prête pas vraiment à discussion, il n'est pas du tout sûr que celle qui décrit le second personnage soit exacte. Il semble plus juste de voir celui-ci en position agenouillée, tourné vers le maître qu'il écoute ou qu'il vénère. Peut-être pourrait-on y voir alors une nouvelle illustration du Vidhurapaṇịta Jātaka, déjà représenté sur des sema du nord-est de la Thaïlande, avec la scène où Vidhura expose la Loi à Punnaka ${ }^{39}$. La face historiée est assez abîmée, contrairement à la seconde face qui est restée plutôt plate (tout comme une des tranches de la stèle), mais la facture du bas-relief ne semble pas avoir été très élaborée. La taille de l'ensemble montre cependant que ce sema était attaché à un lieu de culte important. C'est également le premier exemple de sculpture narrative que nous mentionnons. Nous en retrouverons encore autour de Vientiane.

Quelques autres vestiges aujourd'hui visibles n'ont pas été signalés par P.-M. Gagneux ; ils ont donc probablement été retrouvés après 1977. Parmi eux, une image en pierre du Buddha assis en méditation (l'aspect de la tête procède d'une « restauration ») qui est conservée dans le sanctuaire. À l'extérieur, on peut voir par ailleurs un assemblage curieux, puisqu'au sommet d'une dalle de pierre qui était parallélépipédique, mais dont l'un des côtés semble avoir longtemps servi à affûter des couteaux, est fixée grossièrement, à l'aide d'une sorte de ciment, une antéfixe d'angle en forme de nāga. Les deux pièces sont sculptées dans un grès différent. Si la seconde est évidemment liée à l'art khmer, la première est moins aisée à identifier. On peut penser à un sema non historié, mais la forme qui était semble-t-il très régulière à l'origine laisse également croire à une pièce d'architecture.

L'identité primordialement mône du site est prouvée par le grand sema historié. L'antéfixe apparaît comme un élément isolé qui pourrait avoir été rapporté, peut-être de la zone occupée par le That Luang.

\section{Ban Non Kho}

Dans le village de Ban Non Kho, à 3,5 km de Ban Phon Pa Nao, sur le bord oriental du marais du That Luang, à $1400 \mathrm{~m}$ du bord opposé, a été retrouvé en 1976 un sema d'une hauteur de $117 \mathrm{~cm}$ portant sur ses deux faces unc imagc à pcu près identique du stüpa (fig. 31). La représentation est très sommaire, mais elle est bordée d'un quart de cercle de chaque côté, l'ensemble ressemblant à une tige à laquelle sont attachées deux feuilles (un traitement assez proche avait été remarqué à Ban Nong Khon). La base de la pierre n'est séparée de l'image que par une mince et double bande horizontale. En 1977, ce sema avait déjà été rapporté au Vat Ho Phra Kèo. Les témoignages actuels le font provenir d'un petit tertre d'où émergent des briques. Il correspond très probablement aux ruines qui, selon P.-M. Gagneux, pouvaient être celles d'un ancien monastère lao du $\mathrm{XVIII}^{\mathrm{e}}$ siècle ${ }^{40}$. Dans le même village, à $700 \mathrm{~m}$ au sud-est et toujours au bord du marais, il existe un autre tertre, un peu plus grand, qui recouvre certainement un autre sanctuaire. Il est placé à côté d'un petit « that » en ruine - et de nombreuses pipes en terre cuite, de modèle lao, y ont été retrouvées.

39. S. A. Murphy, The Buddhist Boundary Markers..., op. cit., p. 239-231 (en particulier le sema S591). Puisqu'il s'agit d'un jătaka, Vidhura n'est pas encore le Buddha, mais un bodhisattva.

40. P.-M. Gagneux, Les sites anciens..., op. cit. 


\section{Ban Muang Noy}

Dans le village de Ban Muang Noy, presque en face du temple de Ban Don Koy situé à $2 \mathrm{~km}$ sur le bord opposé du marais, a également été découvert en 1976 un sema de $117 \mathrm{~cm}$ de hauteur, portant sur ses deux faces l'image du stūpa. La facture est supérieure à celle du sema de Ban Non Kho, distant de $2,8 \mathrm{~km}$, et la conservation en est également meilleure. L'image des deux stūpa montre un profil asséz complexe marqué par une base rectangulaire, des divisions dans la hauteur et une flèche qui semble avoir possédé des arêtes. La partie inférieure du sema est nettement séparée de l'image par une large frise horizontale composée de pétales de lotus. Sur le site de la découverte se trouve un tertre avec des briques, correspondant sans doute à un ancien sanctuaire lao. Le sema était déjà déposé au Vat Ho Phra Kèo en 1977.

\section{Ban Dong Phosy}

Le site de Ban Muang Noy est situé à $6 \mathrm{~km}$ au sud-est du Vat Ban Phon Pa Nao. À une distance semblable et en poursuivant exactement dans la même direction, se trouve un vaste terrain, proche du village de Ban Dong Phosy - mais éloigné de $3800 \mathrm{~m}$ de la berge orientale du marais du That Luang - où de grands travaux ont révélé en avril 2010 plusieurs pièces sculptées dans le grès. Celles-ci étaient apparemment réparties, en groupes distincts, sur un espace long de $180 \mathrm{~m}$. Trois de ces groupes formaient semblet-il un ensemble, car ils étaient distants l'un de l'autre de quelques mètres seulement. Les vestiges ont été rapportés rapidement au Vat Ho Phra Kèo, où une partie d'entre eux sont aujourd'hui présentés au public ${ }^{41}$.

Parmi ceux-ci, le plus important est incontestablement un grand sema historié et inscrit, brisé en deux fragments. La partie supérieure, qui mesure $1,55 \mathrm{~m}$, montre que la facture en était très soignée, car elle consiste en une grande stèle plate large de $45 \mathrm{~cm}$, de laquelle se détache, sur une hauteur de $75 \mathrm{~cm}$, l'image d'un personnage assis traitée pratiquement en haut-relief (fig. 32). Le visage de ce personnage s'était apparemment détaché au moment de l'excavation et il a été refixé. On pense davantage à la représentation d'une divinité qu'à celle d'un Buddha, même paré : la tête est entourée et coiffée d'un assemblage d'éléments qui forment une pointe (un mukuta?); sur l'épaule gauche reposent des protubérances, peut-être des parties de la chevelure ; il semble qu'un collier ornait le cou ; le bras droit est replié et la main qui se trouve juste au-dessus de la ceinture tient un objet de forme ovale ; la jambe gauche est clairement repliée à l'horizontale alors que la jambe droite est soit relevée de façon verticale (le genou est alors en contact avec le coude), c'est-à-dire en position royale (mahārājalīlāa), soit pendante (lalitāsana); et le vêtement inférieur paraît plissé, épais et composé de plusieurs pans. Du point de vue du traitement, le modèle iconographique le plus proche est peut-être celui d'un sema historié de Muang Fa Daet qui illustre apparemment une scène du Kulāvaka Jātaka, plaçant au centre Sakka tenant un vajra ${ }^{42}$. Mais les personnages secondaires qui permettraient d'assurer cette identification manquent ici. Au-dessus de cette image sont inscrites huit lignes d'écriture d'aspect très archaïque qu'il n'a pas encore été possible de déchiffrer totalement, mais la référence à un «śri dharmarājā » apparaît clairement

41. Seuls les plus grands blocs sont présentés. La fouille de sauvetage rapide effectuée par le personnel de la Direction générale du patrimoine a permis de collecter de nombreux fragments qui sont actuellement conservés dans les réserves du musée. L'endroit de la découverte est aujourd'hui un terrain de golf.

42. S. A. Murphy, The Buddhist Boundary Markers..., op. cit., p. 210-211. 
(fig. 33) ${ }^{43}$. Deux grosses cassures à la surface de la pierre rendront de toute façon difficile la compréhension du texte, puisque près d'un tiers des caractères, sur les sept premières lignes, ont disparu. L'autre fragment de cette stèle, qui constitue la base, mesure $80 \mathrm{~cm}$ de hauteur (la stèle atteignait donc $2,35 \mathrm{~m}$ ). Sa partie supérieure représente une frise de pétales de lotus sur laquelle le personnage est assis. La forme est légèrement arrondie en arrière et angulaire à l'avant, car le côté postérieur était prolongé par la stèle plate alors que la face antérieure servait de base au personnage en haut-relief. Cette face antérieure, marquée par l'inscription et la représentation d'une scène, focalise l'attention. Il est toutefois important de signaler que la face postérieure, presque totalement cachée par le muret auquel elle s'appuie aujourd'hui, est ornée sur toute sa hauteur de l'image d'un stüpa en épine.

Un autre bloc de grès, long $(2 \mathrm{~m})$ et effilé, constituait semble-t-il un sema, peut-être non achevé. Le sommet est arrondi et porte deux lignes d'inscription dont la gravure a été très peu soignée. La première ligne paraît bien révéler deux mots qui la rattachent à l'épigraphie mône antérieure au $\mathrm{IX}^{\mathrm{e}}$ siècle $^{44}$.

Un troisième sema, dont le côté supérieur est manquant, porte aussi une courte ligne d'inscription, beaucoup plus nette mais incomplète, qui révèle des similitudes avec l'inscription précédente (fig. 34$)^{45}$. Les faces de cette stèle sont plates.

Deux autres pièces au moins ont été historiées ${ }^{46}$ : une base de sema dont il ne subsiste plus que la frise de pétale de lotus et sur laquelle devait être sculpté un Buddha, et la partie supérieure d'un grand et beau sema, haut de $105 \mathrm{~cm}$ et large de $80 \mathrm{~cm}$, dont les faces plates portaient chacune la forme très simple d'un stüpa. La base n'a pas encore été identifiée, mais elle peut figurer parmi les autres vestiges. On peut encore remarquer les deux fragments (parties inférieure et supérieure) d'un sema plat et non historié qui devait mesurer de façon complète $1 \mathrm{~m}$ de hauteur, un autre sema de hauteur semblable et de circonférence presque ovale, enfin un beau bloc de $98 \mathrm{~cm}$ dont on peut considérer qu'il est de forme hexagonale (s'il possède deux faces plates larges de $80 \mathrm{~cm}$ environ, ses deux tranches sont divisées en leur centre par une arête verticale et comptent donc chacune deux côtés).

\section{Ban Somsanouk}

Au début des années 2000 avaient été repérés sur un terrain vague du village de Ban Somsanouk c'est-à-dire à la pointe sud du marais du That Luang, mais dćjà sur son flanc occidental - deux fragments supérieurs de sema portant l'image très stylisée du stüpa.

\section{Ban Don Koy}

Dans l'enceinte du temple du village de Ban Don Koy, sur le bord occidental du marais du That Luang, à la pointe d'une avancée de terre (située pratiquement en face du site de Ban Muang Noy), ont été identifiés en 1975 ou 1976 deux sema portant sur l'une de

43. Ch. Bauer, communication personnelle, 2011. L'écriture ne saurait être postérieure au viII' siècle.

44. Ch. Bauer, communication personnelle, 2011. La première ligne laisse apparaitre wo' et probablement pun $(y a)$ ( «ceci est l'œuvre méritoire »). La seconde ligne, dont pratiquement tous les caractères sont effacés, laisse tout de même apparaître un $\dot{s} r \bar{l}$.

45. On retrouve les mots wo 'et puny $(a)$, plus un mot commençant par pra. Ch. Bauer (communication personnelle, 2011) date pour l'instant cette inscription du VIII' ou IX' siècle.

46. Les pièces historiées étaient plus nombreuses puisque, parmi les fragments de stèle non exposés, figure par exemple le kumbha d'un stūpa. 
leurs faces plates l'image du stīipa ${ }^{47}$. Ces deux vestiges ont été découverts, selon une habitante interrogée récemment, à proximité immédiate, peut-être à côté d'un des étangs rectangulaires dont les larges bords rappellent des trapeang (cf. infra à propos de Ban Saphang Mo) et dont les formes semblent déjà visibles sur la photographie aérienne de 1946. L'un des deux vestiges fut transféré rapidement au Vat Ho Phra Kèo où il est toujours visible. Haut de $82 \mathrm{~cm}$ seulement, sa facture est très basique : l'image du stüpa est traitée dans sa plus simple expression et repose sur une mince frise horizontale faite de pétales de lotus. Le second sema a été coulé dans le ciment qui entoure le sanctuaire et seule la partie supérieure demeure encore visible (P.-M. Gagneux qui l'a vu entièrement lui donnait une hauteur de $1,32 \mathrm{~m}$ ). Elle montre, à la base du stūpa, dont la pointe est aussi traitée de façon très simple, des motifs probablement floraux qui pourraient alors rappeler le sema de Ban Non Kho, tout comme ceux de Ban Saphang Mo et de Ban Simano (cf. infra). Le sanctuaire de Ban Don Koy est entouré d'autres bornes bouddhiques, dont l'une au moins pourrait être la partie inférieure, retournée, d'un sema môn.

\section{Ban Saphang Mo}

Le village de Ban Saphang Mo - qui est aujourd'hui un quartier entièrement intégré à la ville de Vientiane - comporte, comme son nom l'indique, un étang de forme rectangulaire (saphang, du khmer trapeang), parfaitement orienté vers l'est. La présence de ce plan d'eau est surtout visible sur les photographies aériennes de la première moitié du $\mathrm{xx}^{\mathrm{c}}$ siècle, à une époque où la zone était encore très peu occupée. La surface qu'il occupe est importante ( $120 \mathrm{~m}$ sur $90 \mathrm{~m}$ environ) et la largeur des levées de terre qui l'entourent est telle que des bâtiments ont été construits dessus - y compris le temple du village, surélevé, qui occupe tout le côté sud. Cet étang toujours en eau peut être considéré comme l'un des témoignages les plus sûrs d'une forte influence khmère autour du That Luang à un moment donné (cf. infra).

Dans ses environs immédiats, ce sont cependant des vestiges môns qui ont été retrouvés, apparemment tous au même endroit. P.-M. Gagneux signalait déjà, en 1977, la présence à proximité du Vat Saphang Mo d'une stèle (M104) portant sur une face la trace très érodée d'un décor en forme de stüpa. Le croquis qu'il joint à cette description semble indiquer qu'il s'agit du sema ornant un jardin privé, situé à $150 \mathrm{~m}$ au sud du Vat Saphang Mo, où d'autres pièces en grès ont été mises au jour par la suite. À une époque indéterminée, mais antérieure à 2000 , deux blocs de grès correspondant respectivement à un sema non historié de forme assez irrégulière et à un fragment de sema pouvaient déjà être associés au vestige précédemment catalogué. Au début de l'année 2001, deux autres pièces sculptées, découvertes à l'occasion du creusement des fondations d'une maison, purent y être ajoutées : un grand Buddha assis en méditation, amputé de sa tête, mais reposant sur une base solide, et une stèle aux deux faces plates historiées, dont l'une est très abîmée. Ces deux pièces ont rapidement été rapportées au Vat Ho Phra Kèo où elles sont exposées aujourd'hui à proximité du Buddha et de la stèle de Ban Thalat. La première ne se distingue guère des autres Buddha en pierre assis de facture mône, tels qu'on peut les voir dans la plaine de Vientiane : traitement assez fruste, modelé peu marqué, absence de détails, etc. La seconde montre, tout comme les sema du Vat Phon Pa Pao et de Dong Phosy, une iconographie narrative, puisque deux scènes y sont illustrées. La face la plus abîmée ne permet guère de reconnaître plus de deux personnages debout, dont seuls les 
pieds et les jambes subsistent. Ces deux figures apparaissent dos à dos (l'une, positionnée plus bas, pourrait être en avant-plan) et s'en vont peut-être dans une direction opposée, car les jambes semblent esquisser un mouvement. L'autre face présente clairement trois personnages assis, les jambes repliées - l'un, en virrāsana, dominant les deux autres qui paraissent l'entourer et le vénérer, les mains jointes. Les trois figures sont richement habillées, portent des bijoux, et leur tête est ornée d'une coiffure assez complexe, en pointe $(m u k u t a)$. Le traitement est très proche de celui qui caractérise la grande stèle historiée et inscrite de Ban Dong Phosy. Le personnage principal tient d'ailleurs une pose assez semblable, le bras gauche tendu vers la jambe, alors que le bras droit est replié. Une cassure empêche de dire si la main remontant vers la poitrine tenait un objet. Si tel n'était pas le cas, le geste était peut-être celui de l'argumentation (fig. 35$)^{48}$.

À quelques mètres du lieu où furent découverts ces deux vestiges, le propriétaire du terrain de Ban Saphang Mo exhuma une seconde stèle historiée, début 2007, à l'occasion de nouveaux travaux de fondation. Celle-ci présente comme le grand sema de Dong Phosy une iconographie mixte, puisqu'une face montre l'image du stüpa stylisé, entouré dans sa partie inférieure par deux larges feuilles - on retrouve alors un modèle signalé plus haut dans des vestiges géographiquement proches -, tandis que l'autre face décrit une scène où l'on pense reconnaître un homme portant un vêtement qui ne le couvre que de la ceinture au haut des cuisses (du type sampot) : celui-ci fait un geste vers une femme qui porte une jupe longue, peut-être pour lui tendre un objet (fig. 36). Il pourrait s'agir d'une variante de l'épisode de la cour faite à Amarā, dans le Mahosadha Jātaka, qu'on retrouve dans des sema du nord-est de la Thaïlande ${ }^{49}$ - mais la position des personnages serait alors inversée et leur gestuelle différente. La partie supérieure de la stèle est gravée d'une inscription de trois lignes à l'écriture archaïque, présumée mône. La langue ne peut toutefois être identifiée, car aucun mot n'est lisible in extenso.

\section{Le That Luang et ses collections}

La galerie occidentale du That Luang conserve un peu plus d'une soixantaine de vestiges archéologiques en grès, réduits parfois à des fragments. Les pièces appartiennent pratiquement toutes aux domaines khmer et môn, et se distinguent ainsi fortement de celles qui sont conservées dans les deux autres musées religieux de Vientiane (Vat Ho Phra Kèo, Vat Sisaket), pour la plupart postérieures au $\mathrm{XV}^{\mathrm{e}}$ siècle. Cette particularité n'est pas anodine, mais elle n'est pas non plus le résultat d'une répartition organisée entre les différents lieux ${ }^{50}$. On sait que quelques pièces anciennes étaient déjà visibles au That Luang dans la première moitié du $x^{e}$ siècle - ainsi le " Buddha paré » repéré dès 1911 par Henri Parmentier dans l'angle nord-ouest de la galerie, et le « Jaya Bouddha » signalé par Henri Deydier dès le début des années 1950 (cf. infra). En 1977, P.-M. Gagneux rapporte par ailleurs qu'une grande quantité de vestiges anciens avaient servi à construire

48. Ce sema narratif est le seul qui apparaît, pour la plaine de Vientiane, dans la thèse de S. A. Murphy, The Buddhist Boundary Markers..., op. cit., p. 268-269 (S1216). L'insuffisance d'éléments probants ne permet cependant pas d'identifier la scène.

49. S. A. Murphy, The Buddhist Boundary Markers..., op. cit., p. 235-237.

50. Les collections de ces deux temples furent d'abord constituées avec les nombreuses statues lao qui étaient abandonnées dans la ville, après sa destruction par l'armée siamoise en 1828. Au Vat Ho Phra Kèo, des pièces d'autres origines vinrent s'y ajouter à partir des années 1950 et 1960. Les vestiges archéologiques récemment découverts, lorsqu'ils étaient jugés suffisamment intéressants, furent déposés soit au Vat Ho Phra Kèo, soit au Musée historique national. Des musées régionaux commencent également à conserver les vestiges qui sont mis au jour dans les provinces. 
le soubassement du Vat That Luang Nua - temple alors en ruine - et que parmi ceux-ci se trouvaient quelques pièces de facture khmère et lao, mais aussi cinq pièces en grès qu'il rattachait au domaine môn ${ }^{51}$. Il décrit ces dernières et ajoute que « les travaux de déblaiement des ruines du soubassement sont loin d'être achevés et [que] de nouveaux vestiges seront certainement découverts dans les mois qui viennent ${ }^{52}$ ». Il n'y a donc guère de raison de mettre en doute ce qu'indique l'inventaire actuel des collections de la galerie occidentale du That Luang, selon lequel la très grande majorité des vestiges proviennent du Vat That Luang Nua - d'autant que cet inventaire s'avère correct quant à l'origine des pièces entrées avant 1976, comme celles rapportées de Ban Pak Pot, Ban Thin Kèo, Ban Muang Kao, Ban Sapheu, etc. Les vestiges découverts au Vat That Luang Nua forment d'ailleurs un groupe plutôt cohérent, au regard de la facture comme de l'iconographie. Aucun sema n'appartenait à cet ensemble - en tout cas rien qui rappelle les vestiges catégorisés comme tels sur les autres sites. On note en revanche un nombre relativement important d'images du Buddha, puisqu'au moins vingt corps (un seul conserve encore sa tête) sont attestés. Cette caractéristique invite d'ailleurs à s'interroger sur le contexte : la concentration d'un même type de vestiges s'explique-t-elle par la présence d'un atelier (ils auraient alors été fabriqués localement) ou est-elle le résultat d'un rassemblement d'images similaires sur l'important site que couvre l'actuel That Luang?

Les témoignages archéologiques révèlent sans équivoque que la sculpture sur pierre n'était plus en faveur à l'époque du Lān Xāng - la fonte d'images en métal étant devenue, à partir du $x \mathrm{Vl}^{\mathrm{c}}$ siècle, la pratique la plus prisée pour les objets de culte - et même la facture de stèles destinées à être inscrites témoigne d'un appauvrissement certain pour ce qui concerne le mode d'exécution. Il est en outre prouvé que les sema môns ont souvent été réutilisés par les lapicides lao (justement en raison de leur qualité et de la netteté de leur finition), et que le réemploi concernait aussi les images en pierre anciennes (retouchées avec du stuc) - phénomène qui se perpétue d'ailleurs aujourd'hui. Faut-il pour autant croire que toutes les images apparemment retrouvées dans les fondations $\mathrm{du}$ Vat That Luang Nua furent rapportées d'autres endroits, à une époque relativement récente $\left(\mathrm{XVI}^{\mathrm{e}}-\mathrm{XVIII}^{\mathrm{e}}\right.$ siècle) ? Le fait est possible, mais plusieurs raisons tendent à réduire la crédibilité de cette hypothèse. L'une est la relative unité de facture et de matière (un grès rose) de ces sculptures qui montre que leur origine géographique devait être à peu près la même. L'autre est la continuité sur le plan cultuel qui, à l'évidence, a marqué cette zone occupée par le That Luang depuis une époque ancienne jusqu'à aujourd'hui. Une étude précédente a révélé des indices permettant de croire que l'histoire du site est bien antérieure à celle du stūpa révéré par le roi Setthāthirāt au milieu du XVI ${ }^{\mathbb{E}}$ siècle, et qu'un édifice «khmer » en constitue probablement le noyau ${ }^{53}$. Les populations khmérisées - au moins d'un point vue culturel - qui, à une certaine époque, ont laissé des témoignages

51. P.-M. Gagneux, Les sites anciens..., op. cit. Les cinq pièces ont toutes été retrouvées récemment au That Luang, sauf le Buddha assis M5.

52. P.-M. Gagneux, Les sites anciens..., op. cit.

53. M. Lorrillard, "Vientiane au regard de l'archéologie», dans Vientiane, architectures d'une capitale. Traces, formes, structures, projets, op. cit., p. 51-75, en particulier p. 56-64. Outre les sculptures que nous traitons dans la présente contribution, les indices étayant l'hypothèse d'un plan d'occupation organisé selon un modèle khmer sont la grande enceinte en levée de terre, sise devant le That Luang et axée sur celui-ci, ainsi que la présence, enfouie dans le sol de la cour de l'acluel monument, d'une "chaussée " faite de blocs de latérite bien appareillés, laquelle fut dégagée en 1977 à l'occasion de l'installation dans le sol d'un système anti-foudre. Deux étangs ressemblant à des trapeang (nous avons déjà évoqué Saphang Mo) ont par ailleurs été identifiés. 
dans cette partie du bassin inférieur du Mékong n'étaient sans doute guère différentes de celles qui les ont précédées, et que nous avons qualifiées jusqu'à présent de "mônes ». Ce site du That Luang, établi sur une éminence qui domine un large étang, ancien bras du Mékong, n'est peut-être pas le centre cultuel indianisé le plus ancien de toute la région, mais il est probablement celui qui garda le plus longtemps son caractère sacré.

Les images du Buddha assis conservées au That Luang - en tout cas celles dont la hauteur dépasse $30 \mathrm{~cm}$ - constituent un groupe d'une douzaine de pièces environ, toutes incomplètes. Il s'agit de rondes-bosses (même si la face postérieure reste souvent plate) et de hauts-reliefs qui ne diffèrent pas vraiment des quelques autres images retrouvées à Ban Thin Kèo, Ban Nong Khan Khu, Ban Thin Yung (cf. supra) ou de celles conservées dans des temples de la capitale comme le Vat Sisaket, le Vat Kao Yot, le Vat Si Muang et le Vat Dong Palan (cf. infra). Tous ces Buddha sont en position de méditation et en vīrāsana, et ne se distinguent guère que par le socle plus ou moins haut et soigné (dans deux cas particuliers, il s'agit de trois bandes enroulées rappelant le corps du nāga) sur lequel ils reposent, et par la présence éventuelle d'une stèle à laquelle ils s'adossent. Dans tous les cas, la facture demeure assez fruste, le modelé est peu prononcé et les détails sont absents ${ }^{54}$. Ils semblent appartenir à un ensemble assez différent de ceux que constituent les bas-reliefs et les hauts-reliefs des sites rupestres de Vang Sang et de Dan Sung - mais le contraste ne peut être pleinement perçu en l'absence de la quasi-totalité des têtes.

P.-M. Gagneux signalait en 1977 un seul Buddha debout (M3, no actuel TL I/51), découvert au Vat That Luang Nua. L'image est assez endommagée, puisque manquent la tête, la main gauche, le bras droit et les pieds. Deux autres images provenant apparemment du même endroit ont été mises en évidence et conservées par la suite au That Luang : TL I/24 (fig. 37) et TL I/34. Elles sont un peu plus complètes que la première, car subsistent les mains gauches et la moitié des bras droits (la présence de mortaises montre que les avant-bras étaient des pièces ajoutées, tournées vers l'avant), ainsi que la tête pour l'une des images. Celle-ci, bien ronde (bien qu'étant du haut-relief sur stèle) et ornée de grosses boucles - caractéristiques qu'on attribue aux images mônes - ressemble à deux ou trois têtes isolées qui sont également conservées au That Luang, à une tête du Vat Ho Phra Kèo, aux deux têtes du Vat Thin Kèo de Ban Thin Kèo, à la tête de Ban Nong Khan Khu, mais aussi à celle du Buddha de Ban Thalat. Ce dernier devient ici un objet de comparaison, puisqu'il est également figuré debout. Cependant le traitement de son corps est incontestablement plus soigné, notamment par le modclć qui rend parfaitement le galbe des jambes (les images conservées au That Luang sont relativement plates) et par le rendu du vêtement, dont les pans s'avancent avec les bras ${ }^{55}$. Une autre image conservée au Vat Ho Phra Kèo, de même que deux Buddha debout du Vat Inpeng, constituent des exemples à peine différents (cf. infra).

L'aspect de deux torses (c'est-à-dire des parties de corps sans tête, ni bras ni jambes) conservés dans la galerie occidentale du That Luang ne permet pas de déterminer de façon

54. L'absence systématique de soin accordé au traitement des mains est un des èléments montrant que toutes ces images relèvent sans doute d'une même production.

55. Des images de facture comparable (quoique supérieure) sont visibles dans le nord-est de la Thaïlande et relèveraient d'un style Dvāravatī tardif et régional. Elles sont datées du x ${ }^{\circ}$ siècle par Piriya Krairiksh (Rāk Ngao Haeng Silapa Thai, Bangkok, River Books, 2010, p. 271, 283) et Hiram W. Woodward Jr. (communication personnelle). loutefois ce dernier ne remet pas vraiment en question la datation ancienne ( $\mathrm{VIII}{ }^{\mathrm{c}}$ siècle) du Buddha de Ban Thalat et pourrait admettre, si un examen des grès concernés le confirmait, que les images conservées au That Luang soient contemporaines. 
certaine si ces fragments proviennent d'une image assise ou debout ${ }^{56}$. Un troisième torse (TL I/38) appartient quant à lui sans conteste à une image debout, et il est probable que lui était associé un fragment voisin montrant deux pieds posés sur une base carrée avec tenon (TL I/39), elle-même encastrée à présent dans un socle mouluré (TL I/74). Ce corps, brisé au niveau du bas-ventre et de la naissance de la cuisse droite, est remarquable par la partie du sampot plissé qu'il laisse apercevoir, et surtout par sa large ceinture, traitée comme une bande où se succèdent des carrés bordés de perles, entourant en leur centre un motif floral avec bouton et pétales. Le carré frontal (au-dessus du bas-ventre) a plus de relief que les autres (ceux-ci sont davantage gravés dans la pierre que sculptés), comme s'il s'agissait d'un « ceinturon » ou d'une plaque attachée au rebord du sampot par un lien traité en tresses. Il semble bien, vu la trace à peine perceptible, au niveau de la brisure, du relief qui se prolonge sous le « ceinturon ", qu'on avait ici un départ d'ancre. Ce motif, cette fois-ci complet, est d'ailleurs visible si l'on retourne la statue pour examiner sa partie postérieure (fig. 38). Le traitement renvoie alors incontestablement au style du Bayon (fin du XII ${ }^{\mathrm{e}}$-début du $\mathrm{XIII}^{\mathrm{e}}$ siècle), et peut-être plus particulièrement au vêtement (sampot) d'un Avalokiteśvara ${ }^{57}$, probablement à deux bras. Si l'on s'était limité à l'étude du torse, il aurait été relativement facile de confondre cette pièce avec une œuvre mône, même si le grès utilisé est manifestement très différent (plus clair et plus fin) de celui qui a servi pour les pièces qui l'entourent.

Les sculptures conservées dans la galerie occidentale du That Luang qui étaient reconnues jusqu'à présent comme étant de facture khmère (quoiqu'on puisse les qualifier de productions régionales, sous-entendu, d'un art moins évolué que celui des modèles trouvés autour d'Angkor) étaient seulement au nombre de deux - et il s'agissait de pièces d'assez grande dimension, dont le transport était peu aisé. Découvertes à proximité immédiate du That Luang, elles ont probablement été produites pour le site, dont nous avons déjà dit qu'il avait certainement été choisi à date ancienne pour être le lieu de pratiques religieuses.

La date de construction du cloître du That Luang ne peut être établie de façon précise. Il est possible, vu la ressemblance de cette structure avec celle qui entoure le sanctuaire du Vat Sisaket, que sa fondation soit assez tardive et ne remonte qu'au début du XIX $\mathrm{X}^{\mathrm{e}}$ siècle. H. Parmentier, lorsqu'il étudie ce cloître en 1911, relève qu'il « est bien plus mal tracé que la partie centrale [le stūpa] », qu'il est en grande partie détruit, et que " dans [son] angle nord-ouest, une niche, sans doute plus moderne que la galerie ruinée, abrite, sous un élégant fronton qui se détruit par le bas, une statue de Buddha paré debout ; il a les mains unies devant le ventre et tenait dressé un sorte de vajra dont la moitié inférieure est effacée ; il est vêtu d'une tunique, porte une coiffure conique à large diadème, a des bracelets de bras et d'avant-bras, mais pas de boucle d'oreille ${ }^{58} »$. L'architecte, qui ajoute à cette description une photographie (fig. 39), n'indique pas la présence d'un collier, mais il semble bien qu'un tel ornement, visible aujourd'hui (autant que le permet la couche de

56. Dans le cas du premier (TL I/40-1), la main droite complète couvre l'ensemble du ventre, mais sans être associée à un geste précis. D'autres exemples montrent que dans le cas du Buddha méditant, la seconde main (la gauche) pouvait être totalement cachée par la première. L'hypothèse d'un Buddha assis semble donc l'emporter ici. Le second torse (TL I/31), dont la partie inférieure ne descend même pas jusqu'à la limite de la ceinture, pourrait éventuellement être comparé au Buddha debout TL I/24, mais son côté droit ne laisse pas voir, contrairement à son côté gauche, le pan de la tunique. Cette caractéristique se retrouve chez d'autres Buddha assis.

57. Jean Boisselier, Le Cambodge, Paris, Picard, 1966, p. 257-258, en particulier la figure 62c.

58. Henri Parmentier, L'art du Laos, PEFEO n 35, Paris, EFEO, 1954 [publication posthume, rééditée par M. Giteau en 1988], vol. 1, p. 121. 
peinture), était également présent au début du $\mathrm{xx}^{\mathrm{e}}$ siècle et faisait donc partie des attributs d'origine. H. Deydier, qui publie en 1952 une synthèse sur la culture lao, évoque à propos de cette statue un " Bouddha dit de la Fertilité qui [...] n'est que la transformation d'un dvârapâla khmèr (gardien de porte) en Bouddha laotien ${ }^{59}$ ". Il corrige ainsi sensiblement l'appréciation qu'il envoya en 1950 au directeur de l'École française d'Extrême-Orient, puisqu'il avait d'abord considéré que l'image représentait un bodhisattva, peut-être Lokeśvara - tout en livrant des détails qui démentaient en partie cette identification : « [...] mais la masse de laque, d'emplâtre, etc., ne permet pas de distinguer les motifs de la coiffure. D'autre part, par chance pour nous, le dos de la statue est vierge de tout emplâtre et [l']on peut voir le dos d'un magnifique buste khmer. La ceinture est identique à celle représentée dans le $B E F E O$ XL-2, pl. XXXIII-C [il s'agit alors de l'image d'un

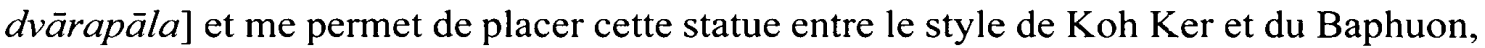
c'est-à-dire les $\mathrm{X}^{\mathrm{e}}$-XI ${ }^{\mathrm{e}}$ siècles A.D. ${ }^{60}$ "). Il n'est malheureusement plus possible d'avoir accès à la partie postérieure de la statue, totalement collée au mur, pour vérifier ces détails ${ }^{61}$. Son emplacement particulier et l'aspect de la niche qui la conservait (cette dernière est encore en très bon état sur la photographie de Parmentier, alors que les murs du cloître étaient totalement ruinés) montrent que la pièce avait été rapportée, mais qu'elle avait sans doute été trouvée à proximité.

Une découverte faite au début de l'année 1951, manifestement dans le même périmètre ${ }^{62}$, a conduit à l'installation, dans la galerie occidentale du That Luang, d'une deuxième image khmère de grande taille : « une statue présumée de Jayavarman VII, relevant de la série des grandes effigies qui figureraient le roi assis en méditation ${ }^{63}$ " (fig. 40). Tout comme un exemple conservé au Musée national de Bangkok ${ }^{64}$, le sampot, rayé, est maintenu par une large ceinture d'orfèvrerie à laquelle sont accrochées des pendeloques. Dans les deux cas, la bande que forme cette ceinture est quasiment identique (cartouches carrés contenant une fleur avec bouton et pétales, bordés sur chaque côté d'une frise faite de perles) à celle qui orne le torse mentionné précédemment (TL I/38) - ce qui renforce la datation proposée pour ce dernier (style du Bayon, fin du XII ${ }^{\mathrm{e}}$-début du XIII ${ }^{\mathrm{e}}$ siècle) et nous conduit à penser que ces deux statues khmères du That Luang étaient parfaitement contemporaines. S'il en est de même pour le $d v \bar{a} r a p a \overline{l a}$, il n'y a malheureusement plus guère d'éléments pour le prouver. Un détail important concernant la "statue présumée de Jayavarman VII » du That Luang est noté par M. Giteau : sa chevelure - contrairement à celles qu'on retrouve sur les statues du même type, bien plus au sud - n'est pas lisse (c'est-à-dire traitée en fines incisions régulières) et terminée par un petit chignon, mais formée de grosses boucles, comme pour le Buddha. L'historienne de l'art explique

59. Henri Deydier, Introduction à la connaissance du Laos, Saigon, Imprimerie française d'outremer, 1952, p. 13.

60. Archives EFEO (« Recherche de documents inédits... » par Jacqueline Filliozat en 1995). Si la ceinture est bien identique à celle qui figure dans le volume cité, elle ressemble davantage, à notre avis, à celle du style d'Angkor Vat (XII' siècle). Cf. J. Boisselier, Le Cambodge, op. cit., p. 255.

61. Il est surprenant que H. Deydier ait pu lui-même observer cette partie, car la photographie de H. Parmentier montre que la statue était déjà profondément engagée dans la niche et qu'elle touchait apparemment le mur.

62. H. Deydier, Introduction..., op. cit.

63. M. Giteau, Art et archéologie du Laos, Paris, Picard, 2001, p. 66.

64. J. Boisselier, La sculpture en Thaïlande, Paris / Friburg, Bibliothèque des arts / Office du livre, 1974, p. 117 ; Steve van Beek \& Luca Invernizzi Tettoni, The Arts of Thailand, Singapore, 2000, Periplus, p. 98. 
cette bizarrerie stylistique en émettant l'hypothèse selon laquelle la coiffure aurait été retravaillée avec un enduit - mais le rendu, soigné et bien proportionné par rapport au reste de la tête, nous conduit à penser qu'il pourrait tout de même s'agir du traitement original. Cette impression est renforcée par la substitution au chignon des Jayavarman VII présumés, et à celle des uṣñ̄ṣa protubérants normalement attendus pour les Buddha, d'une terminaison de la coiffure en une faible pointe. Pierre Baptiste et Thierry Zéphir, qui ont remarqué sur d'autres images du style du Bayon ce même type iconographique, ont proposé d'identifier ces dernières aux Jayabuddhamahānātha (" grands protecteurs Buddha victorieux $»)$ qui sont mentionnés dans l'épigraphie de la fin du $x_{11}{ }^{e}$ siècle $^{65}$. Ceux-ci, au nombre de 23, étaient situés dans autant de villes, dont la plupart paraissent assez excentriques par rapport à Angkor ${ }^{66}$. Est-ce à dire que Vientiane figurait parmi elles? La présence d'une telle image, dont on imagine volontiers qu'elle fut attachée à un monument important, est en tout cas à mettre en relation avec l'existence supposée d'un édifice khmer sous l'actuel That Luang, avec la découverte dans les environs de pièces d'architecture caractéristiques (par exemple, l'antéfixe en forme de nāga du Vat Phon Pa $\mathrm{Nao}$ ), et bien sûr avec la grande enceinte rectangulaire en levée de terre qui marquait cet espace (cf. supra). Les quelques pièces d'art khmer conservées au Vat Ho Phra Kèo et à Luang Prabang permettront peut-être d'apporter quelques indices à ce sujet.

Le grand nombre d'images en pierre du Buddha conservées au That Luang et provenant semble-t-il du même site fait presque oublier la présence de quelques stèles qui ne manquent pourtant pas d'intérêt. Nous mettrons à part le sema TL I/13 qui provient de Vieng Kham (cf. supra), ainsi que deux stèles inscrites en lao (TL I/56 et TL I/3), dont la seconde pourrait également être, à l'origine, un sema môn ${ }^{67}$. La qualité d'exécution de quatre stèles conservées aux portes est et sud du That Luang conduit en effet à s'interroger sur l'identité des sculpteurs de ces dernières. Les deux stèles de la porte orientale n'ont jamais été considérées en fonction de leur support (comme c'est pratiquement toujours le cas dans les études épigraphiques), mais d'après les textes lao relatifs à l'histoire du grand stüpa qui ont été gravés sur leurs faces dans la seconde moitié du Xvi ${ }^{\mathrm{e}}$ siècle $^{68}$. Celles qui, du côté extérieur, entourent la porte méridionale n'ont quant à elles été mentionnées que par P.-M. Gagneux (dans une note non publiée) : il relevait alors, sur l'une des deux, la présence de quelques caractères d'écriture, très effacés, mais qui n'étaient incontestablement ni lao ni tham. Leur graphie très archaïque, confirmée récemment par $\mathrm{Ch}$. Bauer ${ }^{69}$, nous autorise à les rapprocher des épigraphes supposées mônes que nous avons déjà mentionnées, sans toutefois que la notation de cette langue puisse être prouvée,

65. P. Baptiste \& Th. Zéphir, L Art khmer dans les collections du musée Guimet, Paris, éditions de la RMN, 2008, p. 274-275. Je remercie Hedwige Multzer d'avoir attiré mon attention sur cette possible identification. H. W. Woodward ( « The Jayabuddhamahānātha Images of Cambodia », in The Journal of the Walters Art Gallery 52-53, 1994-1995, p. 105-111) propose une autre identification pour ce nom livré par l'épigraphie, celle à Lokeśvara.

66. George Coedès, « La stèle du Práh Khằn d'Aṅkor », BEFEO 41, 1942, p. 255-301, en particulier p. $267,295-296$.

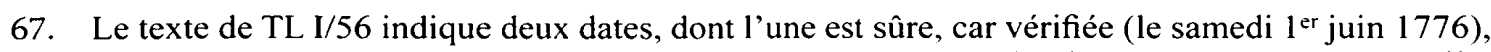
et l'autre, beaucoup plus incertaine en raison d'incohérences (le vendredi $1^{\text {er }}$ décembre 1820). L'effacement presque complet du texte de la face 1 de TL I/3 empêche son déchiffrement ; la face 2 étant pratiquement collée au mur, la lecture de son texte est pour l'instant difficile.

68. M. Lorrillard, « Les inscriptions du That Luang de Vientiane : données nouvelles sur l'histoire d'un stüpa lao », BEFEO 90-91, 2003-2004, p. 289-348.

69. Communication personnelle, 2011. 
car la lecture certaine d'un mot complet s'avère impossible. Les quatre stèles ont en tout cas été façonnées dans le même grès, et leur facture, très soignée, est identique. Chacune d'elles possède des tranches non plates mais biseautées, c'est-à-dire présentant deux plans. La surface de la stèle non gravée de la porte sud a été réduite de moitié, puisqu'il ne subsiste plus du matériau initial que son côté droit, après une brisure longitudinale dans l'axe central de la pierre.

\section{Vientiane}

Le site qui fut choisi pour être la capitale lao au $X V l^{\mathrm{c}}$ siècle, mais dont on sait qu'il avait déjà une histoire plus ancienne ${ }^{70}$, a laissé peu de vestiges attestant les marques des cultures mône et khmère. L'absence de quantité est toutefois compensée par la qualité de certaines pièces - la difficulté consiste alors à savoir si celles-ci ont été rapportées ou si elles ont vraiment été produites sur place.

Mis à part le That Luang (qui était situé en dehors de la première enceinte), seul un site de la ville pourrait éventuellement révéler les vestiges architecturaux d'un édifice khmer : le Vat Si Muang. Le Vat Inpeng conserve quant à lui des pièces cultuelles très anciennes dont la taille incite à penser qu'elles sont attachées au site depuis fort longtemps. Elles peuvent d'ailleurs être mises en relation avec des vestiges moins importants situés à proximité. Le Vat Ho Phra Kèo donne à voir quelques pièces d'art khmer d'origine souvent inconnue. Les autres vestiges sont des pièces isolées et de qualité médiocre qui sont conservées dans quelques temples, où ils ont probablement été rapportés.

\section{Le Vat Si Muang}

Le Vat Si Muang a été à peine signalé par H. Parmentier dans L'art du Laos. En 1911, le sanctuaire était en reconstruction et l'architecte mentionnait seulement l'« autel considérable de l'ancienne pagode avec, au milieu, un prisme en latérite de $2 \mathrm{~m}$ de haut et de $0 \mathrm{~m} 60$ de côté ». Il notait également : «En arrière de la pagode, restes d'énorme that ruiné à plan carré redenté ${ }^{11} "$.

Pour ce dernier édifice, il omet de préciser des détails importants : d'abord le fait que le matériau de construction soit uniquement constitué de blocs de latérite - dont la plupart ont été parfaitement taillés, de façon à obtenir des jointures impeccables; ensuite le plan rectangulaire, surprenant dans le cas d'un stüpa; enfin l'orientation parfaite vers l'est, alors que le sanctuaire lao est tourné vers le sud. En l'absence de véritable étude architecturale de ce monument, il est difficile de se livrer à des conjectures à son sujet. Son identification à un stūpa lao paraît tout de même problématique et il est utile de rappeler que la latérite était, avec le grès, un matériau de construction privilégié des anciens Khmers.

C'est cet édifice - présenté comme un « temple en ruine »-que H. Deydier propose de voir comme l'emplacement d'origine du «prisme de latérite » de H. Parmentier, identifié alors comme " un ancien linga ${ }^{72}$ ». Il est peu aisé aujourd'hui, vu la difficulté d'accès à ce bloc de pierre - placé au centre de l'autel, habillé de bandes de tissus et entouré de quantité d'autres objets de culte - de se faire une idée exacte de sa fonction originelle. Les arêtes nettes de ses quatre côtés font cependant douter de l'interprétation donnée par H. Deydier. Dans la tradition populaire, il n'est d'ailleurs pas associé à un sivaling

70. M. Lorrillard, « Vientiane et le Mékong... », op. cit., p. 33-50.

71. H. Parmentier, L 'art du Laos, op. cit., p. 114.

72. H. Deydier, Introduction..., op. cit., p. 102. 
(linga de Śiva), mais à un lak muang, c'est-à-dire à un pilier de fondation (de la ville). L'association implicite qui était faite avec un vestige khmer peut toutefois être fondée.

Sur le même autel que le lak muang est aujourd'hui visible une pièce en grès qui ne semble pas avoir été mentionnée dans les études précédentes (elle a, semble-t-il, échappé à l'attention de H. Parmentier, de H. Deydier, de M. Giteau et de P.-M. Gagneux), alors que par sa facture elle relève clairement de l'art khmer (fig. 41). Il s'agit manifestement d'un Buddha paré, très proche par son traitement de celui qui a été photographié dans les années 1940 au Vat Sangkhalok de Luang Prabang et qui est aujourd'hui conservé dans le musée de la ville. Cette dernière pièce est datée du début du XII ${ }^{\mathrm{e}}$ siècle. Une autre image en grès disposée sur l'autel, que l'obscurité de l'endroit rend difficilement visible, est apparemment identifiable à un Buddha assis, sans tête, qui était adossé à un nāga dont on perçoit encore la partie inférieure du corps. Étant donné sa facture, cette pièce relèverait également de l'art khmer.

Deux fragments de personnages sculptés en pierre provenant du Vat Si Muang, ou de sa proximité immédiate, sont actuellement conservés au Vat Ho Phra Kèo. Le premier (HPK I/69) est un torse masculin à quatre bras découvert peu avant 1951 à quelques centaines de mètres du temple (fig. 42) ${ }^{73}$. M. Giteau en donne une description précise, mais ne peut identifier le personnage en l'absence de la tête et des attributs que pouvaient brandir les mains. Les éléments de parure (collier, brassards, vêtement, ceinture) suffisent cependant à associer l'image au style d'Angkor Vat (première moitié du XII ${ }^{\mathrm{e}}$ siècle) ${ }^{74}$. La facture est très soignée et il s'agit probablement de la plus belle pièce khmère retrouvée dans la plaine de Vientiane. La seconde image se limite à une tête masculine découverte apparemment au Vat Si Muang même, à une date qui doit se situer entre 1951 et 1968. Malgré le caractère très « érodé » de la pièce qui lui donne un côté assez brut, M. Giteau la rattache au style du Bayon ${ }^{75}$.

\section{Le Vat Inpeng et ses environs}

Le Vat Inpeng a sans doute joué très tôt un rôle essentiel dans le schéma d'implantation des espaces sacrés, puisque son angle sud-ouest jouxtait l'enceinte de la ville, dans sa partie amont, à l'endroit où le mur fortifié suivait le cours de la Nam Passak. Il apparaît donc comme un temple placé géographiquement à une extrémité, et c'est d'ailleurs de sa porte orientale, située quasiment dans l'axe longitudinal du sanctuaire, que commençait l'ancienne voie parallèle à la berge du Mékong - cette « seconde ligne de pagodes » que mettait particulièrement en évidence $\mathrm{H}$. Parmentier et qui comprend d'autres grands temples comme les Vat(s) Ongteu, Hay Sok, Mixai, Sisaket et Ho Phra Kèo. La fin de cette ligne, en aval, était marquée par le Vat Si Muang - ce qui n'était peut-être pas fortuit. L'importance du Vat Inpeng est également révélée par la surface du domaine qui lui fut originellement attribué (apparemment la plus grande de tous les temples de la ville), puisque les cartes françaises du début du $x x^{\mathrm{C}}$ siècle montrent qu'à cette époque il s'étendait très largement vers le nord, avec un espace où se trouvaient les ruines d'un deuxième sanctuaire (très vite rasé et oublié). C'est le tracé d'une grande artère ouvrant

73. H. Deydier, Introduction..., op. cit., p. 13.

74. M. Giteau, Laos. Étude de collections..., op. cit., p. 67, pièce 413. Au vu du caractère particulier du costume et des parures, Th. Zéphir (communication personnelle) propose, avec une grande prudence, car il n'a pas étudié la statue originale, une datation un peu plus tardive. On soulignera ici que M. Giteau attribue au style du Bayon, et même franchement au début du XIII ${ }^{\mathrm{e}}$ siècle, toutes les autres statues khmères conservées au Vat Ho Phra Kèo.

75. M. Giteau, Laos. Étude de collections..., op. cit., p. 69, pièce 450. 
davantage la capitale vers les villages situés en amont, au-delà de la muraille, qui, autour de 1912, amputa de moitié le domaine initial du temple. H. Parmentier reconnaît de facto le statut privilégié de ce temple en lui consacrant une description assez longue. C'est également - semble-t-il - le seul endroit de la ville où il relève des pièces qui lui paraissent d'origine khmère. Ce « détail »n'a plus vraiment été mis en évidence par la suite, alors qu'il se révèle peut-être capital pour retracer l'histoire la plus ancienne de Vientiane.

Parmi les pièces qui retiennent l'attention de $\mathrm{H}$. Parmentier se trouve une statue en grès schisteux qui a été transformée par l'addition tardive d'enduits. Ces traces de modification amènent l'architecte à douter que le sujet d'origine ait vraiment été un Buddha, mais la description qu'il donne - «c'est une image debout, le bras gauche descendant le long du corps ; le bras droit est replié et la main droite relevée près du sein a trois doigts étendus, le pouce et l'index se touchant par l'extrémité $[\ldots]^{76} \gg$ - ainsi que la connaissance que nous avons aujourd'hui des sculptures bouddhiques de type Dvāravatī, font immanquablement penser à une image en vitarkamudrā. Cette impression est renforcée par une photographie de H. Parmentier montrant en premier plan la partie supérieure du profil de la sculpture (fig. 43), et est totalement confirmée par l'observation de l'image elle-même, toujours conservée au Vat Inpeng (VIP/28), même si les derniers travaux de "restauration » ont modifié encore plus son aspect original ${ }^{77}$. Il en va de même pour une seconde image du Buddha en pierre du Vat Inpeng (VIP/25) que Parmentier oublie de mentionner dans son texte, mais qui figure bien aux côtés de la première sur sa photographie. Cette omission est peut-être due à l'aspect plus grossier de la facture, mais la similitude des drapés et de la représentation générale montre de façon incontestable que les deux sculptures sont contemporaines et relèvent du même art ${ }^{78}$. Elles peuvent être rapprochées d'au moins deux des Buddha debout de la galerie occidentale du That Luang (TL I/24 et TL I/34). Leur datation est pour l'instant difficile : le $\mathrm{X}^{\mathrm{e}}$ siècle a été proposé ${ }^{79}-$ mais le XIII ${ }^{\mathrm{e}}$ siècle pourrait également être retenu ${ }^{80}$.

À la suite de la description de la statue en grès, H. Parmentier rapporte : « Sur le même autel est une curieuse pierre en dalle à quatre faces qui me paraît également d'origine cambodgienne. Une des faces larges présente onze figures : en bas une figure couchée, puis trois rangs de trois buddhas attestant la terre ; ils sont sans bijoux et ont l'uṣnịș̣a-chignon ordinaire, mais pas d'ürñā ; un dernier, en haut, est plus important ; tous sont abrités dans des niches à décor flammé. L'autre grande face présente en bas un motif indistinct, au-dessus le même nombre et la même disposition dc bodhisattvas, les mains dans le giron et portant de nombreux bijoux. Sur chaque petite face, quatre buddhas attestent la terre. Cette pièce a son tenon trop grand fiché dans le montant d'une base carrée qui peut être également khmère ${ }^{81}$ ". La photographie qui montrait déjà les deux Buddha debout en pierre fait aussi apparaître clairement cette " pierre en dalle » fichée sur un socle qui semble-t-il n'est pas le sien, la face visible étant celle où sont sculptés les bodhisattva

\section{H. Parmentier, L'art du Laos, op. cit., p. 101.}

77. En restaurant la main droite, par exemple, le geste de la vitarkamudrā est devenu celui de l'abhayamudrä, mais les traces de l'index rejoignant le pouce restent malgré tout visibles.

78. Les photographies prises à différentes époques montrent que les « restaurateurs » ont d'abord restitué un avant-bras en abhayamudră, et ont modifié plus récemment la tête.

79. H. W. Woodward Jr., communication personnelle.

80. Th. Zéphir, communication personnelle (avec la réserve qui s'impose, car la pièce n'a pu être directement étudiée).

81. H. Parmentier, ibid. 
en méditation. H. Parmentier oublie certains détails que montre encore la pierre toujours disposée sur l'autel - ainsi la présence, entre quelques-uns des Buddha attestant la terre, de petites divinités venant s'insérer dans des espaces que ne remplissent pas les décorations des niches. Une autre photographie ancienne (fig. 44) montre que seule la tête du Buddha couché était restée préservée, ce qui est encore à peine le cas aujourd'hui.

Il semble bien qu'il faille dater cette description du premier texte de 1911, car une note de bas de page mentionne à propos de la pierre en dalle qu' " Elle est à cette heure renversée ", ce qui reporte cette précision à une constatation faite ultérieurement, probablement en 1927. H. Parmentier en profite alors pour ajouter la description d'une pièce qu'il n'avait peut-être pas remarquée lors de son premier séjour : "à côté est une autre pierre à quatre faces, mi-carrée, mi-ronde, avec deux niches à buddha superposées sous arcs flammés ; la pièce pose sur un piédestal khmèr à emboîtement, avec cuve à ablutions dont le bec est cassé ". Ces deux vestiges associés sont parfaitement visibles sur une photographie prise par Hiram W. Woodward Jr. en 1965 (fig. 45) ${ }^{82}$, et l'on se rend bien compte qu'à l'inverse de ce qu'écrivait H. Parmentier en 1911, c'est le piédestal qui est cette fois trop grand pour la pierre à quatre faces. Il y eut manifestement une redistribution des bases par la suite, puisque la sculpture la plus haute $(1,30 \mathrm{~m})$ et la plus richement traitée repose désormais sur le « piédestal à emboîtement », alors que la plus petite $(75 \mathrm{~cm})$ est encastrée dans un socle moins haut et peu mouluré. Il est toutefois à peu près certain qu'aucune de ces deux bases n'était à l'origine associée aux objets qu'elles supportent aujourd'hui ${ }^{83}$.

H. Parmentier utilisait à propos de ces sculptures la formule « curieuse pierre en dalle à quatre faces » qui montre à quel point leur type ne lui était pas familier. M. Giteau les qualifie de «bornes bouddhiques » sculptées d'images du Buddha paré et précise que «Par leur diadème très évasé et par leur couvre-chignon conique, ces images sont dans la tradition du style d'Angkor Vat : mais par leur attitude assise, les genoux très écartés, ainsi que par les arcatures de feuillage qui les abritent, elles appartiennent nettement à l'art de Lopburi, l'art khmer de la Thaïlande ; elles sont certainement postérieures au style d'Angkor Vat ${ }^{84}{ }^{\prime \prime}$.

Si la paire de Buddha debout devait être attribuée au $x^{\mathrm{e}}$ siècle, nous aurions alors entre celle-ci et les bornes bouddhiques - une différence de datation de plus de deux

82. Je le remercie de m'avoir communiqué ce document inédit.

83. Si l'on distingue bien les restes d'une protubérance sur l'un des côtés de la partie supérieure du " piédestal à emboîtement » (le terme est ici inapproprié puisque la pièce est faite d'un seul bloc, et non d'éléments séparés), il n'est pas du tout sûr qu'il s'agisse du bec verseur proprement dit, mais plutôt de la corniche qui aurait contribué à le soutenir. On ne peut donc parler non plus d'une cuve à ablutions (élément distinct qui aurait disparu ?). La borne sculptée, avec ses cinq étages de représentations, ne jure pas vraiment avec son piédestal actuel, mais il faut remarquer que les deux pièces ont à peu près la même largeur (environ $45 \mathrm{~cm}$ ), ce qui n'est pas logique. Leur assemblage a, par ailleurs, été facilité par un épais raccord en ciment. Il est sans doute utile de rappeler le point de vue de J. Boisselier, selon lequel « les piédestaux des images du Buddha [...] ne comportent pas, en principe, de cuves à ablutions » (Le Cambodge, op. cit., p. 216). L'incompatibilité de l'autre base avec la sculpture à laquelle on l'a fixée (à l'aide de ciment) est plus évidente encore, puisque la seconde n'est pas centrée sur la première. On voit sur l'autel deux autres blocs de grès, de forme circulaire, et pourvus de sortes de mortaises creusées dans leur partie centrale. Il est possible qu'il s'agisse de socles, mais il est alors peu probable qu'ils aient été mieux adaptés à nos sculptures.

84. M. Giteau, Art et archéologie du Laos, op. cit., p. 65. J. Boissclicr (Le Cambodge, op. cit., p. 99) parle également de «bornes bouddhiques » pour des pièces qui apparaîtraient à partir du XII ${ }^{\mathrm{e}}$ siècle, mais il classe ces dernières dans la catégorie plus large des caitya, un qualificatif que les historiens de l'art emploient davantage. 
siècles et un renvoi à deux périodes historiques et culturelles bien distinctes. Cette divergence dans la chronologie des témoignages est curieuse, mais elle n'est pas forcément à exclure. Le manque d'unité dans les collections du Vat Inpeng est de toute façon souligné par la présence d'autres vestiges encore non signalés : des sema, dont deux au moins se rattachent très clairement aux collections de pièces mônes signalées plus haut. L'un d'eux ( $1 \mathrm{~m}$ de hauteur visible) laisse encore voir sur l'une de ses faces l'image stylisée du stüpa, comportant à sa base la forme du kumbha et reposant sur une frise de pétales de lotus. Un autre sema, beaucoup plus grand (1,95 $\mathrm{m}$ de hauteur visible) et non historié, fait penser par sa forme et la couleur de son grès aux quatre stèles que nous avons mentionnées autour des portes orientales et méridionales du That Luang.

Deux autres vestiges donnent à croire, par leur forme parallélépipédique, qu'ils pourraient être identifiés à des éléments d'architecture ancienne. C'est le cas pour un bloc de grès rose scellé de façon verticale dans une dalle, mais plus encore pour un grand bloc de grès gris planté sur une pelouse ( $1,90 \mathrm{~m}$ de hauteur visible, $75 \mathrm{~cm}$ de largeur), lui-même entouré de gros blocs de latérite qui ont dû appartenir à une construction ${ }^{85}$.

À ces vestiges conservés au Vat Inpeng, il faut sans doute en associer deux autres dont la situation géographique est très proche (respectivement à $150 \mathrm{~m}$ et $200 \mathrm{~m}$ ), de même que certaines caractéristiques. Le premier est un gros piédestal (ou socle) en grès rose sis dans le domaine du Vat Hay Sok. Il est en partie enterré, ce qui ne permet pas de visualiser la totalité de ses moulures. Il est certain en tout cas qu'il servait de base à une pièce importante. On a posé dessus deux dalles de grès qui empêchent de voir la mortaise, ronde ou carrée. De ces deux dalles, l'une a nettement la forme d'une stèle, l'autre peut avoir été une pièce d'architecture déformée par l'aiguisage répété d'instruments en métal. Le second vestige facilement identifiable est un sema avec image du stüpa, de facture très fruste, laissé à l'abandon dans le domaine du Vat Mixai. Ces deux types de vestiges ne sont pas visibles ailleurs dans la ville ancienne ${ }^{86}$ - ce qui devrait nous amener à accorder une attention particulière à ce périmètre, qu'on pourrait encore élargir de quelque $200 \mathrm{~m}$ vers l'est jusqu'aux domaines des anciens Vat(s) Si Bun Yeun et Kang. Le premier a livré deux Buddha en pierre debout et une tête qui ont été apportés au Vat Ho Phra Kèo (cf. infra pour le plus grand d'entre eux) ${ }^{87}$; dans le second se trouvait une belle stèle historiée qui est aujourd'hui conservée au Musée historique national : haute de $76 \mathrm{~cm}$ et large de $46 \mathrm{~cm}$, de forme ogivale, elle présente sur ses deux faces une divinité fémininc debout, les bras écartés, positionnée dans une niche qui possède de chaque côté une base sur laquelle repose un décor en arcature ${ }^{88}$. Le style est à l'évidence khmer et fait penser à des antéfixes de l'art du Bayon.

85. Il faut peut-être considérer avec un ceil nouveau certains commentaires de $\mathrm{H}$. Parmentier sur les particularités architecturales du Vat Inpeng - comme sa description de la grande porte orientale de l'enceinte ( $L$ 'art du Laos, op. cit., p. 101), qui lui faisait penser à deux demi-édifices, ainsi qu'il en avait relevés en Indonésie.

86. Exception faite, pour les sema avec stüpa, d'une petite pièce (dont la partie supérieure est manquante), qui a été trouvée au Vat Si Amphone. Elle est maintenant conservée au Vat Ho Phra Kèo.

87. P.-M. Gagneux, Les sites anciens..., op. cit., pièces M10, M13, M19. Les deux premières n'ont pu encore être repérées.

88. P.-M. Gagneux, Les sites anciens..., op. cit., pièce M60. Il est indiqué au musèe historique que cette sculpture provient de Ban Si Khai, dans la province de Savannakhet, ce qui est évidemment une erreur. 


\section{Les collections du Vat Ho Phra Kèo}

Nous avons déjà mentionné que deux sculptures reconnues comme procédant de l'art khmer avaient été retrouvées autour du Vat Si Muang et déposées au Vat Ho Phra Kèo. Ce temple transformé en musée contient quelques autres pièces relevant du même art qu'il est utile de signaler ici.

L'une d'entre elles est célèbre : il s'agit de la fameuse stèle à quatre faces inscrites découverte en 1902 à Say Fong, à une vingtaine de kilomètres au sud-est de Vientiane, par Georges Maspéro, administrateur des services civils de l'Indochine. L'article qu'il a publié en 1903 dans le Bulletin de l'École française d'Extrême-Orient est à l'origine d'un « mythe » qui ressurgit régulièrement dans les synthèses historiques sur l'Asie du Sud-Est, à savoir que le village de Say Fong aurait pu être construit sur une ancienne ville khmère ${ }^{89}$. Il nous suffira de rappeler ici que l'histoire de cette localité ne peut être séparée de celle de Vieng Khuk, gros bourg situé sur la rive opposée (aujourd'hui thaïlandaise) du Mékong - et que ce muang à cheval sur le fleuve (à l'endroit où ce dernier achève la grande boucle orientée vers le sud qui, après Vientiane, interrompt son cours ordinaire vers l'est) était, durant la période faste du Lān Xāng (XVI ${ }^{\mathrm{e}}-\mathrm{XVIII}{ }^{\mathrm{e}}$ siècle), une très importante place commerciale où aboutissaient les routes terrestres venant du Siam, notamment d'Ayuthya. Les restes d'importants édifices religieux y ont donc été retrouvés, mais ils sont tous d'origine lao. Aucune trace de construction ou même de structure khmère n'a pu y être relevée ${ }^{90}$. G. Maspéro reste très vague sur la localisation exacte de la petite stèle en écriture khmère qu'il a signalée pour la première fois, mais les notes et une photographie de H. Parmentier, datées de 1911, montrent qu'elle se trouvait dans les ruines du temple de Ban Si Than Tai, à 2 km au nord-est de Say Fong ${ }^{91}$ (fig. 46). La véritable origine de cette stèle reste problématique. Louis Finot, qui l'a traduite très vite, a montré qu'il s'agissait d'un des édits en langue sanskrite commandés par Jayavarman VII afin de commémorer la construction d'hôpitaux et de sanctuaires sur le territoire qu'il dominait ${ }^{92}$. Les édifices sacrés étaient bâtis en dur sur un même modèle, et les deux exemples les plus septentrionaux qu'on a identifiés jusqu'à présent sont ceux qu'on a déjà mentionnés (cf. introduction) dans les provinces thaïlandaises de Sakorn Nakhon (Prasat Phan Na) et d'Udon Thani (Prasat Ku Kaeo Ban Chit) ${ }^{93}$, à une centaine de kilomètres environ, à vol d'oiseau, de Vientiane. Comme les édits n'y ont pas été retrouvés, on peut suggérer - avec les réserves qui s'imposent - que l'un d'eux a été rapporté à Say Fong, peut-être par la route de Nong Han. On peut également chercher, dans une proximité plus

89. Georges Maspéro, «Say-Fong, une ville morte », BEFEO 3/1, 1903, p. 1-17. Pour les extrapolations erronées auxquelles a conduit cette découverte, cf. M. Lorrillard, « D’Angkor au Lān Xāng : une révision des jugements ", Aséanie 7, 2001, p. 19-34.

90. H. Parmentier (L'art du Laos, op. cit., p. 125) signale bien au Vat Xethaphon de Vieng Khuk « une antéfixe d'angle khmère grossière, et un dvārapāla avec massue », mais il poursuit en rappelant qu' "On ne signale cependant l'existence d'aucune ruine khmère dans les environs ». Les deux pièces signalées par l'architecte sont toujours sur place et semblent toutes deux être des antéfixes. La première paraît figurer la face d'un lion. Quelques petites sculptures du Buddha en grès sont conservées dans le même temple (Vat Thep Phon actuel). L'une au moins, adossée à une stèle incomplète (terminée probablement en tête de $n \bar{a} g a)$, pourrait être de facture khmère, sinon mône.

91. H. Parmentier, L'art du Laos, op. cit., p. 127.

92. Louis Finot, « L’inscription sanskrite de Say-Fong » (Notes d'épigraphie, II), BEFEO 3/1, 1903, p. 18-33.

93. Muang Boran Journal 30/3, 2004 (en thaï). Plusieurs contributions traitent des arogväśāla du nord-est de la Thaïlande. 
immédiate, un site supposé khmer à l'origine et qui aurait ensuite été recouvert par une structure lao. Le That Luang, qui a livré entre autres vestiges deux statues du style du Bayon, dont une image proche des portraits de Jayavarman VII, pourrait correspondre à ce type de lieu ${ }^{94}$.

G. Maspéro, avant même d'évoquer la petite stèle de Say Fong, donne la description d'une statue : "Celle-ci, en grès fin, d'un gris verdâtre, haute d'environ $0 \mathrm{~m} 40$ et large de $0 \mathrm{~m} \mathrm{30}$, représente une divinité assise, les jambes repliées, la plante des pieds tournée vers le ciel, les mains croisées sur les genoux, la paume en l'air. La physionomie est douce, la face ronde est plate, le nez droit et large, les arcs sourciliers proéminents, la bouche grande, les lèvres fortes. La coiffure est une sorte de bonnet conique garni d'une couronne à ornement du genre perle. Cette statue porte à sa base un tenon qui devait la maintenir sur un autel ou un motif architectural. Elle appartient manifestement à l'art brahmanique qui a décoré Angkor ${ }^{95} »$. On ne saurait dire, à lire l'administrateur, si la statue fut trouvée au même endroit que la stèle. Cela se vérifie cependant quelques années plus tard dans une courte mention d'H. Parmentier (cf. infra), ainsi que sur la photographie mentionnée précédemment. C'est ce vieux cliché qui permet précisément d'identifier la sculpture de Say Fong avec celle qui est aujourd'hui conservée au Vat Ho Phra Kèo sous le $n^{\circ}$ HPK I/73, dont la provenance est encore dite inconnue. M. Giteau parle à son propos d'un " personnage assis en vìrāsana dont les deux mains posées sur les genoux sont brisées. Visage rond aux yeux largement ouverts; expression souriante. Diadème orné de denticules ; couvre-chignon orné de trois rangs de pétales de lotus. Le costume est un sampot plissé, drapé avec un bord rabattu sur le devant ${ }^{96}$ ". Elle le date de la fin du style du Bayon, soit du début du XIII ${ }^{\mathrm{e}}$ siècle. Aucun rapport n'a été établi entre cette sculpture et la stèle de Say Fong, car l'historienne de l'art ignorait que les deux pièces provenaient toutes deux du temple de Ban Si Than Tai. Elle décrit toutefois une autre image de personnage assis, provenant de Say Fong et de dimensions à peu près égales : «Visage partiellement refait en ciment. Diadème décoré de fleurs, de perlage ; couvrechignon orné de rangs de pétales de lotus. Le costume n'a pas été sculpté ${ }^{97}$ ». Ces caractéristiques nous empêchent de confondre cette image avec la précédente, même si elle est également associée à l'art du Bayon et datée du début du X III $^{\mathrm{e}}$ siècle. La description est trop rapide, puisque la position des mains et des jambes n'est pas précisée - et l'on se demande finalement si ce n'est pas cette seconde image que M. Giteau identifiait à celle décrite par G. Maspéro. La difficulté est accrue par le fait que cette sculpture semble avoir disparu. Deux hypothèses quant à sa provenance restent toutefois possibles. Dans son unique phrase consacrée au temple de Ban Si Than Tai, H. Parmentier rapporte qu'il a vu, « outre la petite stèle khmère signalée par G. Maspéro, deux statues de cet art, l'une avec la ceinture, le mukuṭa à diadème et cône caractéristique ${ }^{98} \gg$. Aussi l'autre, qu'il s'abstient de décrire ${ }^{99}$, était-elle peut-être la statue dont M. Giteau écrivait que le costume n'avait pas été sculpté. La seconde hypothèse résulte d'une note que G. Maspéro insère dans son

94. On rappellera une nouvelle fois la mise au jour, lors de travaux menés en 1977 dans la cour du monument, d'une chaussée axiale en blocs de latérite parfaitement appareillés. Cf. M. Lorrillard, «Vientiane au regard de l'archéologie », op. cit.

95. G. Maspéro, « Say-Fong... », op. cit., p. 3.

96. M. Giteau, Laos. Étude de collections..., op. cit., p. 68. L'image est référencée sous le numéro 438.

97. M. Giteau, Laos. Étude de collections..., op. cit., p. 67-68. L'image est référencée sous le numéro 434.

98. H. Parmentier, L'art du Laos, op. cit., p. 127.

99. H. Parmentier ne semble pas avoir photographié cette seconde image. 
article, après avoir décrit la statue associée à la stèle : «J'ai découvert une autre statue de même pierre, de même facture, et certainement de même origine dans une des pagodes qui se trouvent au sud de la pagode de Vat-sop (Viêng-chan) [on comprend qu'elle doit être située à une dizaine de kilomètres au nord-ouest de Say Fong, et qu'elle peut déjà être considérée comme appartenant au domaine de ce village] ; c'est un Çiva, dans la pose classique, accroupi, un genou horizontal au sol, l'autre dressé ; il tient ses mains sur chacun de ses genoux, la paume en l'air ${ }^{100}$ ». Mais on va bientôt voir que la position des bras et des mains donne également la possibilité d'identifier cette sculpture à une autre image conservée au Vat Ho Phra Kèo.

On ne s'est pas encore interrogé sur l'identité de cette (ces) sculpture(s) qui fu(ren)t découverte(s) sur le même lieu que la stèle de Say Fong. Le texte de l'inscription fait lui-même référence à trois divinités bouddhiques mahayaniques honorées : le Buddha Bhaișajyasugata et les bodhisattva Sūryavairocana et Candravairocana. Est-il envisageable que l'image HPK I/73 du Vat Ho Phra Kèo soit identifiée à l'une d'entre elles ?

Parmi la petite collection de sculptures khmères du Vat Ho Phra Kèo figure une image de provenance inconnue qui répond en partie à la description faite par G. Maspéro d'une image découverte à quelques kilomètres en amont de Say Fong (cf. supra). Il s'agit de la pièce cataloguée HPK I/71 (fig. 47), que M. Giteau présente ainsi : «Personnage assis devant une stèle [...]. Il manque la partie supérieure de la stèle, la coiffure, le visage, le genou gauche. Le visage a été refait en ciment. Personnage assis, genou gauche replié, genou droit relevé. Costume drapé avec un bord rabattu et une ceinture à pendeloques. Fin de l'art du Bayon, XIII ${ }^{\mathrm{e}}$ siècle ${ }^{101} »$. Deux détails suffisent toutefois à mettre en doute la correspondance entre les deux pièces : l'omission par G. Maspéro d'une stèle à laquelle s'appuie la statue, et la mention des paumes en l'air, ce qui n'est pas le cas pour au moins une des mains de la pièce du Vat Ho Phra Kèo, l'autre ayant été cassée.

Il faut en tout cas rapprocher cette statue d'une autre pièce en grès de provenance inconnue qui est conservée au Vat Ho Phra Kèo sous la référence HPK I/65 (fig. 48), car elle lui est semblable dans sa disposition générale - quoique dans le détail elles apparaissent très distinctes. M. Giteau la décrit ainsi : " Personnage assis devant une stèle, genou gauche replié, genou droit relevé. Le nez du personnage ainsi que l'attribut qu'il tenait dans sa main droite et qu'une partie de son socle sont brisés. Visage ovale aux arcades sourcilières jointives ; coiffure comprenant un diadème orné d'un perlage et trois chignons couverts de rangs de pétales de lotus. Des boutons de lotus parent les oreilles. Le costume découvre l'épaule droite et laisse voir le pectoral qui n'a pas été ciselé. Un pan plié est posé sur l'épaule gauche ; le vêtement inférieur est drapé avec un double pan. Art khmer, fin de l'art du Bayon, XIII $^{\mathrm{e}}$ siècle ${ }^{102}$ ». Les différences de traitement n'empêchent pas de penser que les deux sculptures pourraient représenter une même divinité, car une particularité iconographique de la seconde paraît restituable sur la première (traces sur le côté gauche de la tête) : le traitement de la chevelure en trois chignons (triśikhā). Avec une certaine prudence, Th. Zéphir a proposé récemment de voir dans une divinité masculine d'un type très proche, conservée au musée Guimet (MG 18135), une représentation du bodhisattva Mañjuśrī ${ }^{103}$. C'est cette identification que nous retiendrons également pour nos deux images.

100. G. Maspéro, « Say-Fong... », op. cit., p. 3, n. 3.

101. M. Giteau, Laos. Étude de collections..., op. cit., p. 68. L'image est référencée sous le numéro 440.

102. M. Giteau, Laos. Étude de collections..., op. cit., p. 67. L'image est référencée sous le numéro 415.

103. P. Baptiste \& Th. Zéphir, op. cit..., L'Art khmer.., op. cit., p. 290. 
On peut toutefois se demander - étant donné la rareté apparente de ce type d'image (J. Boisselier et Th. Zéphir n'en citent qu'un exemple), la difficulté qu'il y a à les identifier et le manque d'informations sur leur origine - si HPK I/65 et HPK l/71 ne proviennent pas plutôt de Vat Phu et de sa région, tout comme d'autres images qui ont été conservées un temps au Vat Ho Phra Kèo avant d'être transportées au musée de la Révolution ${ }^{104}$. On trouve en effet dans le Sud-Laos plusieurs statues très semblables, mais dans un état plus dégradé ${ }^{105}$. Il ne serait donc guère surprenant que ce soient ces exemples les mieux préservés qui aient été déplacés vers le principal musée de la capitale.

Une des rares sculptures khmères du Vat Ho Phra Kèo qui puisse être identifiée de façon certaine est un corps - sans tête et sans bras - d'un Vajrapāṇi / Vajradhara, catalogué sous le $n^{\circ}$ HPK I/67 (fig. 49). M. Giteau en donne la description suivante : « [...] assis en vīrāsana et en vajrahumkaramudrā sur un socle orné d'un rang de pétales de lotus. Costume lisse. Art khmer ; style du Bayon, fin $\mathrm{XII}^{\mathrm{e}}$-début $\mathrm{XIII}^{\circ}$ siècle ${ }^{106} »$. La facture est quasiment identique à celle d'une image un peu plus abîmée qui est conservée au Vat Visun de Luang Prabang. Les représentations de Vajrapāṇi sont généralement liées à des sanctuaires d'hôpitaux de Jayavarman VII. Deux statues complètes de cette divinité du bouddhisme mahāyāna ont été retrouvées dans les hôpitaux de Ku Kaeo (province de Khon Kaen) et de Ku Santarat (province de Maha Sarakham), situés respectivement à 170 et à $250 \mathrm{~km}$ environ de Vientiane. Leur facture est similaire à celle du Vajrapāṇi conservé au Vat Ho Phra Kèo.

Une image un peu moins visible actuellement, car repoussée dans une zone de réserve sur le côté droit de l'autel du Vat Ho Phra Kèo, est une divinité à quatre bras assise devant une stèle, dont la provenance ne semble pas tout à fait assurée. Selon M. Giteau, qui la date de la fin de l'art du Bayon, "ses mains ont été manifestement retaillées. Sans doute ce personnage était-il primitivement un Avalokiteśvara, tel qu'on en trouve un nombre important au Cambodge ; par la suite cette image manifestement mahayanique a été mutilée de ses attributs et, devant son chignon, la figure du Buddha en samādhi a été bûchée ${ }^{107}$ ».

Une dernière image de facture assurément khmère, mais manifestement non achevée, consiste en un corps en grès, sans tête et sans avant-bras, portant la référence HPK I/66. Cette statue est absente de l'inventaire de M. Giteau et il est fort probable qu'elle soit entrée au musée après 1968. Avec cette pièce, il semble qu'on se situe également dans l'art du Bayon, vu qu'elle dispose, au niveau du nombril, d'une première large bande horizontale qui devait correspondre à un bord rabattu (recouvrant alors la ceinture), d'une seconde bande en dessous qui peut avoir été prévue pour la gravure de pendeloques, et enfin d'une double chute en ancre aux contours géométriques. On distingue toutefois une partie en relief qui peut apparaître comme un drapé en poche étendu de la pointe de la hanche gauche à la cuisse droite, comme on en relève plutôt dans le style d'Angkor Vat. On est tenté de voir dans cette statue l'image d'un dvārapāla: une bande verticale en réserve sur l'abdomen pouvant alors avoir été prévue pour figurer une arme.

104. C'est le cas par exemple d'un très beau Ganeśa découvert à Muang Khong, dans la province de Champassak.

105. Trois d'entre elles sont exposées au musée de Vat Phu ; les autres sont conservées dans sa réserve et le dépôt de Champassak.

106. M. Giteau, Laos. Étude de collections..., op. cit., p. 69. L'image est référencée sous le numéro 444.

107. M. Giteau, Art et archéologie du Laos, op. cit., p. 65. Cf. également M. Giteau, Laos. Étude de collections..., op. cit., p. 67. L'image est référencée sous le numéro 416. Elle est sculptée dans un grès jaune et dite provenir du Vat That Noi, un monastère disparu qui se trouvait à l'est de la ville (cf. P.-M. Gagneux, Les sites anciens..., op. cit., pièce Ml6). 
Parmi les pièces en grès de facture plus fruste qui sont actuellement conservées à l'intérieur du musée du Vat Ho Phra Kèo, on signalera également deux têtes de Buddha (HPK I/52 et HPK I/50) de provenance inconnue qui, par leur chevelure traitée en grosses boucles, évoquent clairement, pour M. Giteau, l'art môn de Dvāravatīi ${ }^{108}$; une autre tête provenant du That Luang (HPK I/59) qui semble être du même type, bien que très abîmée ${ }^{109}$; une image de personnage debout (probablement le Buddha), dont le bas du corps manque à partir des genoux et dont la réalisation semble en être restée à l'état d'ébauche ${ }^{110}$; enfin, un Buddha debout de provenance inconnue, sur lequel M. Giteau ne décèle plus que des échos de l'art de Dvāravatī ${ }^{111}$. Il est vrai qu'on retrouve dans le visage et dans le drapé de cette statue les caractéristiques tardives qu'on avait notées à Dan Sung et à Vang Sang, mais aussi chez les Buddha debout du Vat Inpeng et peut-être chez ceux du That Luang.

C'est cet art de transition entre une facture mône et une marque plus proprement khmère qu'on décèle également sur deux stèles bouddhiques historiées, conservées dans le péristyle du Vat Ho Phra Kèo, et de provenances inconnues. La première (HPK I/26), de forme ogivale et d'une hauteur dépassant légèrement $1 \mathrm{~m}$, présente trois Buddha assis en méditation, d'apparence semblable - même si celui du centre est placé en arrière-plan par rapport aux deux autres, probablement par souci de composition (fig. 50). M. Giteau observe que «sur ces images, les influences de l'art de Dvāravatī sont déjà fort estompées ${ }^{112}$ ». Les associations de Buddha ne sont pas rares dans les productions des $\mathrm{XII}^{\mathrm{e}}$ et $\mathrm{XIII}^{\mathrm{e}}$ siècles trouvées en Thaïlande et au Cambodge. Elles apparaissent cependant surtout sur des plaques en terre cuite. Le fait que les images de la stèle du Vat Ho Phra Kèo soient limitées à trois a conduit $\mathrm{M}$. Giteau à les identifier avec les prédécesseurs du Buddha historique Gotama : Kakusanḍha, Konagāmana et Kassapa ${ }^{113}$. Une autre identification possible, proposée pour des images au traitement proche (pas de signes particuliers) qui sont plutôt en māravijaya, renvoie à Gotama, au futur Buddha Metteya et au Baișajyaguru ${ }^{114}$.

La seconde stèle historiée, un peu plus petite $(70 \mathrm{~cm}$ de hauteur pour $58 \mathrm{~cm}$ de largeur) et plus abîmée que la précédente, de forme également moins élancée (avec une partie supérieure plus ronde), se situe de façon plus certaine dans le registre iconographique proprement mahayanique (fig. 51). Il s'agit là manifestement d'une " triade bouddhique » - la seule qu'on connaîtrait alors à Vientiane - représentant le Buddha, Lokeśvara à sa droite et Prajñāpāramitā à sa gauche. Les quatre bras et la particularité de la coiffure du second ne peuvent être absolument attestés, car la pierre semble avoir été en partie bûchée et ce sont les têtes qui ont le plus souffert. Cette représentation est plus grande que la plupart des sculptures de triades bouddhiques qui ont été retrouvées et dont le transport était plus aisé. Cette taille relativement importante est peut-être significative

108. M. Giteau, Art et archéologie du Laos, op. cit., p. 60. Les pièces sont référencées sous les numéros 422 et 427 .

109. M. Giteau, Laos. Étude de collections..., op. cit., p. 62. L'image est référencée sous le numéro 448.

110. Selon P.-M. Gagneux (Les sites anciens..., op. cit.), cette image (M19 pour l'auteur), référencée sous le numéro 411, provient du Vat Si Bun Yeun.

111. M. Giteau, Art et archéologie du Laos, op. cit., p. 60. L'image est référencée sous le numéro 428.

112. M. Giteau, ibid.

113. M. Giteau, Art et archéologie du Laos, op. cit., p. 61.

114. Piriya Krairiksh, Rāk Ngao Haeng Silapa Thai, op. cit., p. 371 -372. L’image identifiée à Metteya occupe alors la position centrale et sa taille est supérieure à celle des autres. 
quant à l'existence d'un atelier local. On reste apparemment dans le style du Bayon, fin du XII ${ }^{\mathrm{e}}$ siècle ou début du XIII ${ }^{\mathrm{e}}$ siècle.

\section{Les autres vestiges conservés dans la ville}

On ne fera guère que mentionner les quelques autres images anciennes conservées dans des temples de Vientiane, car leur apparence assez fruste et leur manque d'originalité par rapport aux vestiges déjà mentionnés ne leur confèrent pas une importance particulière.

Le Vat Sisaket possède une très riche collection d'images du Buddha, la plupart en bronze et presque toutes de facture lao ${ }^{115}$. 11 est toutefois possible de dénicher parmi elles quelques images beaucoup plus anciennes, en grès. L'une d'elles est un Buddha en méditation dépourvu de sa partie supérieure et dont la base, fait notable, révèle en faible relief la forme d'une tête, très érodée (SSK I/586). Cette image avait déjà été relevée par P.-M. Gagneux en 1977 (M49). La facture est médiocre et ressemble fort à celle des Buddha assis présumés môns conservés au That Luang. Une autre image du Buddha en méditation procède d'une exécution plus habile et semble se rattacher à l'art khmer angkorien, en raison du capuchon de nāga qui le recouvre (il ne reste plus que quatre têtes sur sept) et du trône représentant le corps reptilien lové. La statue porte des enduits de stuc qui dénaturent un peu sa forme originelle, particulièrement au niveau du visage dont les traits semblent avoir été assez prononcés. Une autre pièce, confinée au fond du sanctuaire et passée inaperçue jusqu'à présent, date probablement, elle aussi, de l'époque angkorienne. Il s'agit d'une tête, sans doute masculine, dont les yeux paraissent fermés et qui ressemble par son allure générale (absence de traitement de la coiffure, traits épais) à la tête masculine du Vat Si Muang conservée aujourd'hui au Vat Ho Phra Kèo, que M. Giteau associait à l'art du Bayon.

Le Vat Kao Yot, qui faisait également partie des temples de la ville lao ancienne, conservait déjà en 1975 une image du Buddha en méditation adossé à une stèle, proche par sa facture des images reconnues comme mônes qui sont conservées au That Luang ${ }^{116}$. La tête a subsisté et montre un uṣṇ̄ṣa assez important, comme c'est le cas sur la plupart des autres images complètes.

P.-M. Gagneux rapportait en 1977, à propos du Vat Dong Palan, qu'à l'occasion des travaux de reconstruction de ce temple, une dizaine d'années plus tôt, trois vestiges anciens en pierre avaient été découverts ${ }^{117}$. Il semble que seuls deux de ceux-ci soient toujours conservés dans le sanctuaire. Le premier est une image du Buddha en méditation adossé à une stèle et installé sur une base presque cylindrique, qu'orne une double frise en pétales de lotus. Le second ressemble davantage à une stèle sur laquelle on a sculpté en très bas-relief une image du Buddha assis en māravijaya / bhümisparśamudrā, posture peu fréquente dans la sculpture très ancienne et dont nous avons déjà vu une représentation surprenante dans le temple principal de Ban Muang Kao. Dans le cas présent, en raison du faible modelé, la main droite ne touche pas vraiment le sol. Le sculpteur a pourtant essayé, en allongeant démesurément le bras le long de la cuisse, de figurer la position. La facture est extrêmement fruste, même si l'on discerne les traces d'un trône et d'un nimbe. Enfin, le sanctuaire conserve bien encore un fragment de stèle plate sur laquelle

115. Parmi les images de facture lao, les autres matériaux utilisés sont le bois, la brique enduite de stuc, la terre cuite et la résine recouverte de feuilles de métal. Les plus anciennes images en bronze (fin $X V^{e}$-début $X V I^{e}$ siècle) proviennent manifestement du Lān Nā.

116. P.-M. Gagneux, Les sites anciens..., op. cit., pièce M2.

117. P.-M. Gagneux, Les sites anciens..., op. cit., pièces M99, M100 et M103. 
a été gravée, sans doute bien après la réalisation du support, une inscription lao - mais il est peu probable qu'on puisse l'identifier à ce que P.-M. Gagneux supposait être un fragment de linga, de section octogonale, avec une partie supérieure arrondie. Dans les trois cas, les descriptions nous ramènent quoi qu'il en soit à des vestiges de facture mône. Notons que le quartier de Dong Palan, situé hors de la première enceinte de la ville lao, était jusqu'au milieu du $\mathrm{xx}^{\mathrm{e}}$ siècle - comme son nom l'indique - un espace de verdure entouré de zones humides. Il n'est donc pas étonnant d'y retrouver des traces d'occupation anciennes.

Si l'on n'était familier des résultats surprenants auxquels aboutissent certains travaux de « restauration », on ne pourrait guère reconnaître, en regardant deux statues argentées confinées dans la partie arrière du sanctuaire du Vat Nak, deux images du Buddha très anciennes. P.-M. Gagneux indique qu'elles furent retrouvées autour de 1960 - au moment de la restauration du temple - et que leur apparence originelle avait été modifiée avec du ciment ${ }^{118}$. Hautes toutes deux d'un peu plus d' $1 \mathrm{~m}$, elles ont un caractère très fruste qui n'incite guère à leur accorder de la considération. En faisant abstraction des têtes qui ont été totalement remodelées, on pourra toutefois retrouver les caractères généraux des Buddha assis môns qui ont été identifiés ailleurs, en particulier la position des jambes et la présence d'une stèle à laquelle l'image s'adosse. L'une des statues est cn méditation. L'autre cst en bhümisparśamudrā, mais il est possible, comme le suggère P.-M. Gagneux, que la main droite orientée vers le sol soit le résultat d'une retouche. Le Vat Nak est situé dans l'espace que limite la seconde enceinte de la ville lao ancienne, prolongeant cette dernière sur plus d' $1 \mathrm{~km}$ vers l'aval. Dans le même périmètre, au Vat Si Amphone (également appelé Vat Nak Noi), avait été retrouvé avant 1977 un fragment de petit sema à quatre faces, portant sur chacune d'entre elles l'image du stūpa. Ce vestige, aujourd'hui conservé au Vat Ho Phra Kèo, conforte l'hypothèse d'une occupation mône dans cet espace.

Les alentours immédiats de Vientiane n'ont pas encore révélé d'autres vestiges anciens, mais il est probable qu'avec l'accroissement rapide et continuel qui caractérise la capitale depuis une vingtaine d'années, d'autres vestiges seront mis au jour.

Nous dépasserions trop le cadre de cette étude si nous reprenions dans le détail la description de la douzaine de sites voisins établis sur la rive droite du Mékong (Thaïlande) qui comportent des vestiges anciens - le plus éloigné d'entre eux n'étant qu'à une quarantaine de kilomètres au sud-ouest de la capitale lao ${ }^{119}$. Il est en tout cas certain que ces implantations étaient en rapport direct avec celles que nous avons relevées dans la plaine de Vientiane. Par la quantité, la taille et la qualité de facture des vestiges qu'ils recèlent, les sites regroupés autour de Phu Phra Bat pourraient d'ailleurs apparaître comme le foyer culturel dominant de toute cette région, du moins pour ce qui relève de l'occupation ou de l'influence mône. Il est possible que le centre de gravité se soit déplacé vers Vientiane sous l'effet d'une influence khmère croissante autour du $\mathrm{XII}^{\mathrm{e}}$ siècle, car, pour cette période, les témoignages s'avèrent plus significatifs sur la rive gauche.

118. P.-M. Gagneux, Les sites anciens..., op. cit., pièces M101 et M102.

119. La plupart de ces sites ont été notés sur notre carte de la plaine de Vientiane (cf. supra). Des informations les concernant ont déjà été signalées dans M. Lorrillard, "Vientiane et le Mékong... ", $o p$. cit., p. 42-43. Un article spécifique sur les sites historiques des provinces thaïlandaises septentrionales du plateau de Khorat est également en cours de rédaction. On consultera par ailleurs S. A. Murphy, The Buddhist Boundary Markers..., op. cit. 


\section{Le confluent de la Nam Ngum}

Si le confluent de la Nam Ngum n'est situé qu'à $55 \mathrm{~km}$ de Vientiane à vol d'oiseau, il en est en revanche distant de près d'une centaine de kilomètres par la voie du fleuve, car celle-ci est marquée par de nombreux et larges méandres. Nous avons vu que sur les rives de la Nam Ngum, les sites môns anciens étaient concentrés dans la partie septentrionale de la plaine de Vientiane et qu'aucun vestige significatif n'avait encore été retrouvé en aval de Ban Vieng Kham. Le confluent de la Nam Ngum semble pourtant avoir été attractif pour les sociétés anciennes, puisqu'en plus d'importants sites lao découverts à proximité - comme l'ancien Muang Pak Huay Luang (actuel Phon Phixay, sur la rive droite) -, quelques témoignages môns ont été retrouvés dans ses environs immédiats. Le plus en aval se trouve à un peu plus d'une quinzaine de kilomètres de Pak Ngum, dans le village de Ban Pengchan, sur la rive droite du Mékong (Thaïlande) ${ }^{120}$. Il s'agit d'un beau et grand sema avec image du stūpa, dont la forme est très particulière puisqu'il se présente comme une sorte de tige très fine reposant sur un globe (le kumbha?), qui prend lui-même appui sur une frise de motifs associés au lotus. Ce vestige apparaît comme marginal, non seulement par son traitement stylistique, mais aussi et surtout par sa position, puisque c'est le dernier à avoir été identifié sur les rives du Mékong, dans cette région. Pour retrouver un nouvel ensemble de sema, il faudra ensuite redescendre le fleuve sur près de 300 km, jusqu'au confluent de la Sé Bang Fai.

En amont du confluent de la Nam Ngum, à une douzaine de kilomètres de distance, on signalera un sema pratiquement accolé au sanctuaire de Ban Thun Lua. L'image du stūpa n'a été sculptée que sur une seule face : très simple, elle montre une forme en épine.

À quelque $10 \mathrm{~km}$ au sud-ouest, toujours au bord du Mékong, on a pu apprendre récemment qu'un temple lao en ruine de Ban Tha Dua - le Vat Si Hom - a été édifié au tout début du XVII ${ }^{\mathrm{e}}$ siècle, comme l'atteste le texte (inédit) d'une longue inscription. Celle-ci a été gravée sur une belle stèle dont la forme nous est familière, et qui est probablement d'époque antérieure. Cette hypothèse est confortée par la présence, au milieu des ruines, d'un socle ou d'un piédestal mouluré qui indique une facture remontant apparemment à la période angkorienne. On trouve également sur le site de nombreux blocs de latérite qui pourraient appartenir à une construction de la même époque.

Plus en amont encore, presque à égale distance de Pak Ngum et de Vientiane, P.-M. Gagneux rapporte qu'il avait mis au jour en 1973, dans les ruines du Vat Som Hong de Ban Simano, une stèle de facture ancienne avec un décor en forme de stüpa très stylisé ${ }^{121}$. Ce sema portait en outre dans sa partie supérieure une inscription lao de huit lignes relativement récente. Il n'est pratiquement plus visible aujourd'hui, puisqu'au cours de la reconstruction du temple, il fut décidé de le sceller dans la chape de ciment qui constitue le sol de l'intérieur du sanctuaire. Aussi émerge-t-il à peine du carrelage qui a été posé par la suite, et il n'est plus possible de distinguer son décor originel. Un croquis de P.-M. Gagneux (M81) montre toutefois que le stüpa était sculpté de façon semblable sur les deux faces et qu'il présentait une forme en épine avec deux « feuilles » se développant autour en quart de cercle, comme sur certains modèles que nous avons remarqués autour de l'étang du That Luang.

120. Hleng Boranakhadi Prathet Thai, Lem 3 [Sites archéologiques en Thaïlande, vol. 3], Krom Silpakorn, Bangkok, 2532 (1989), p. 58-60. Une observation récente de la seconde face a révélé quc celle-ci porte également l'image du stūpa posé sur le khumba, entouré de deux feuilles de lotus.

121. P.-M. Gagneux, Les sites anciens..., op. cit. 


\section{Le confluent de la Nam Thon}

Les sema découverts à Ban Nong Khon (cf. supra) ont montré qu'il existait très probablement un lien entre les sites môns riverains de la Nam Ngum, établis dans la partie septentrionale de la plaine de Vientiane, et le bassin de la Nam Thon, à l'ouest de la chaîne Say Phu Pha Nang, par le biais d'une passe facilement accessible. Au confluent de cette rivière avec le Mékong, à Ban Pak Thon, à une quarantaine de kilomètres en amont de Vientiane, ont été remarqués deux blocs de grès taillés qui, par leur forme, sont identifiables à des sema anciens - même si aucun d'eux ne porte l'image du stüpa (fig. 52).

\section{Le confluent de la Nam Mi}

La plaine de Vientiane marque une limite particulière dans l'expansion septentrionale des sociétés indianisées anciennes sur le territoire lao actuel, car il n'existe plus, au-delà de cet espace, de surface plane à la superficie aussi vaste, en tout cas sur la rive gauche du Mékong. Cette dernière est dès lors marquée par la présence d'importants reliefs auxquels le fleuve quittant la Chine se heurte, et au travers desquels il a parfois réussi à creuser son lit. La répartition des témoignages archéologiques lao avait déjà montré qu'il existait, pour l'époque du Lān Xāng, un véritable hiatus dans la densité des zones habitées entre Luang Prabang et Vientiane ${ }^{122}$. Un seul site a révélé des témoignages significatifs (notamment des inscriptions) en nombre relativement important : l'ancien muang à cheval sur les deux rives qui est à l'origine des deux villes actuelles de Sanakham (Laos) et de Chiang Khan (Thaïlande). La seconde devait apparemment son importance à la grande plaine qui s'ouvre vers le sud, passage privilégié pour se rapprocher du bassin de la Ménam Chao Phraya. Mais la géographie met encore davantage en évidence le rôle passé de la première, puisqu'elle est établie au confluent de la Nam Mi dont la vallée, semblable à une longue faille de direction nord-sud, fut sans doute un itinéraire terrestre privilégié : cette dernière offrait en effet un chemin nettement plus court que la voie du Mékong, au moins jusqu'à un certain point, pour remonter jusqu'à Luang Prabang. D'ailleurs c'est bien à Sanakham qu'ont été identifiés les vestiges les plus anciens : les inscriptions lao sur stèle sont antérieures à celles qui ont été découvertes à Chiang Khan. L'une d'entre elles, conservée au Vat Si Phum et datée de 1545, est pour l'instant le plus vieil exemple attesté de sema môn réemployé à l'époque lao. L'image caractéristique du stūpa est visible sur ses deux faces. Elle est complète sur l'un des côtés et montre un traitement beaucoup plus élaboré que ceux qui ont pu être relevés sur les sema de la plaine de Vientiane, à quelque $150 \mathrm{~km}$ en aval (fig. 53) ${ }^{123}$. Le kumbha, posé sur une base rectangulaire, y est vraiment représenté comme un pot, et au-dessus de lui s'élève toute une série de motifs qui donnent à l'ensemble une certaine élégance. Il est pratiquement certain que l'autre côté fut sculpté de la même façon, mais il n'en reste plus que la partie inférieure (kumbha) et la pointe finale, le reste ayant été entièrement bûché et aplani pour y graver le texte lao. Le modèle stylistique de ce sema est parfaitement identifiable, puisque plus d'une trentaine d'exemples très similaires ont été découverts à une soixantaine de kilomètres au sud, dans le district thaïlandais de Wang Saphung (province de Lœi), en particulier à Ban Bung Phak Kam, que la Nam Lœi relie au Mékong. Cet espace est cependant luimême assez isolé - puisqu'il constitue l'extrême limite occidentale des sema - et il reste encore à rechercher dans les sites plus orientaux les types iconographiques originaux.

122. M. Lorrillard, «Pour une géographie historique... », op. cit., p. 147-150.

123. Bien que relativement éloignée de la capitale lao, la ville de Sanakham appartient encore à la province de Vientiane. 
D'autres vestiges anciens ont été retrouvés à Sanakham, notamment au Vat Niai Pha Hot. Celui-ci se distingue d'abord par la présence, dans le sanctuaire, d'une paroi rocheuse de quelque $6 \mathrm{~m}$ de hauteur (!) sur laquelle est sculptée une image du Buddha debout, dont les bras démesurément longs viennent se croiser au niveau du bas-ventre. Cette représentation est probablement le résultat de multiples retouches et il est difficile de se faire une opinion sur son origine. Autour d'elle sont cependant disposées d'autres images, parmi lesquelles trois sculptures en pierre dont l'antiquité paraît plus assurée. La première est un Buddha méditant adossé à une stèle. On ne peut distinguer clairement ce qui appartient à la facture originale et ce qui résulte de retouches. D'une façon générale, l'image nous rappelle fortement les Buddha môns conservés à Vientiane, ce qui n'est guère surprenant étant donné la présence du sema avec stūpa mentionné précédemment. Le meilleur rendu du modelé du visage, de même que la forme de la bordure de la jupe et la hauteur de la coiffure nous conduisent néanmoins à nous interroger sur une influence possible de l'art du Cambodge ou de Lopburi. C'est bien une facture de type khmer qu'il faut en tout cas reconnaître dans les deux autres images du Buddha protégé par Mucilinda qui sont conservées à côté (fig. 54). Si celles-ci présentent des traits communs, comme le traitement du nāga qui est caractéristique des œuvres du XII ${ }^{\mathbb{E}}$ siècle (surtout pour ce qui concerne la forme du capuchon et des sept têtes), elles montrent également dans leur apparence générale des différences très sensibles, notamment dans les visages et les coiffures. Les retouches lao, hormis l'épaisse couche de peinture dorée qui recouvre ces images, ne paraissent guère importantes. La plus grande statue relève par le traitement de sa tête et par sa coiffure (apparemment un diadème associé au couvre-chignon) du type iconographique des Buddha parés, même si elle ne semble pas garder la trace des bijoux ornant le corps, comme le collier pectoral, les brassards et les bracelets.

L'examen des vestiges historiques les plus anciens de la plaine de Vientiane montre un contexte archéologique bien distinct de celui que nous avions mis en évidence dans une précédente contribution relative aux provinces du Sud et du Centre-Laos. La différence la plus flagrante est l'absence totale de vestiges khmers préangkoriens, puisque leur limite la plus haute se situe à quelque $400 \mathrm{~km}$ en aval de l'actuelle capitale lao, à la hauteur de l'embouchure de la Sé Bang Fai. Le Mékong, dont on avait souligné le rôle fondamental pour les populations des régions méridionales, n'est plus au-dessus du $17^{\mathrm{e}}$ degré de latitude Nord qu'une voie de communication parmi d'autres. Elle est même souvent la plus longue et la plus difficile, les chaînes de reliefs guidant son orientation et réduisant considérablement les surfaces habitables.

Les recherches préhistoriques avaient déjà montré l'importance de la partie septentrionale du plateau de Khorat, en particulier des terres basses du bassin de la Nam Songkhram (ou de Sakorn Nakhon), où une quantité exceptionnelle de sites datant de l'âge des métaux ont été identifiés. La plaine de Vientiane constitue d'un point du vue physique, au-delà du fleuve, la prolongation et l'extrémité naturelles de cet espace. De façon évidente, différents types de cultures s'y sont diffusés par le biais de routes terrestres, mais également via de petits affluents orientés soit vers le nord (sur la rive droite), soit vers le sud (sur la rive gauche). Par bien des aspects, l'espace triangulaire que circonscrivent les massifs du Say Phu Pha Nang, du Say Phu Pha Daeng et du Phu Khao Khuay constitue une sorte d'enclave finale, une des dernières zones où les grandes dynamiques migratoires du premier millénaire se sont arrêtées. 
Les enquêtes de surface effectuées entre 1974 et 1977 et au cours des dernières années ont mis en évidence, dans la plaine de Vientiane, une trentaine de sites possédant des vestiges historiques antérieurs à l'occupation lao. Elles ont surtout révélé une véritable cohérence géographique, puisque le choix de ces lieux semble avoir toujours été déterminé par des conditions écologiques précises. Nous avons relevé l'importance de plusieurs bassins - en particulier celui de la Nam Ngum qui paraît avoir bénéficié d'une grande faveur - et de certaines configurations physiques comme celle du grand étang du That Luang et de la colline qui le domine. Ici l'implantation humaine pourrait bien avoir précédé celle de la partie de la ville de Vientiane qui est directement riveraine du Mékong, pourtant considérée jusqu'à présent comme la plus ancienne.

L'identité ethnique des populations qui laissèrent des vestiges historiques - il s'agit toujours de témoignages du culte du bouddhisme - constitue un problème délicat, même si des données nouvelles ont permis de préciser un certain nombre de points. La présence mône est bien attestée dans la plaine de Vientiane et semble être ancienne. Elle est prouvée non seulement par les témoignages linguistiques que livrent des inscriptions, mais également par le grand nombre de vestiges au style et à la facture caractéristiques. Malgré le lien que constitue le Mékong, un rapport direct entre les témoignages môns de la plaine de Vientiane et ceux de la plaine de Savannakhet ne peut être véritablement ćtabli. Ccux-ci procèdent de deux groupes distincts, rattachés chacun à des ensembles géographiquement différenciés, auxquels appartiennent en revanche des sites du nord-est de la Thaïlande. Les vestiges septentrionaux pourraient d'ailleurs être plus tardifs que les vestiges méridionaux - plus proches d'un foyer originel de diffusion, si celui-ci est situé à Muang Fa Daet comme cela est généralement admis. La difficulté qu'on a, en Thaïlande, à reconnaître en certains vestiges l'appartenance à une aire culturelle précise, qu'elle soit mône ou khmère, se retrouve au Laos pour une période qu'on situe autour du $\mathrm{x}^{\mathrm{C}}$ siècle. Jusqu'à présent, il a été commode d'employer, pour les témoignages relevant de cette époque, l'étiquette d'" art de Lopburi ». Celle-ci, extrêmement vague et très réductrice, masque en fait le caractère complexe des multiples productions régionales affiliées à l'art proprement angkorien. Des sites rupestres comme ceux de Vang Sang et de Dan Sung fournissent sur cette question des données très importantes. La culture angkorienne tardive a exercé une influence plus reconnaissable pour ce qui concerne des pièces découvertes dans la capitale laotienne. Il apparaît en effet certain que par le biais du bassin de Sakorn Nakhon, une influence khmère véhiculant des modèles directement issus du centre de l'Empire a franchi le Mékong entre le $\mathrm{XI}^{\mathrm{e}}$ et le XIII' ${ }^{\mathrm{e}}$ siècle, pour atteindre la région de Vientiane. Il reste à en mesurer l'intensité et surtout à définir dans quelle proportion cet apport a pu pénétrer la culture tai-lao qui commence à s'implanter dans ce même espace au début du second millénaire. 


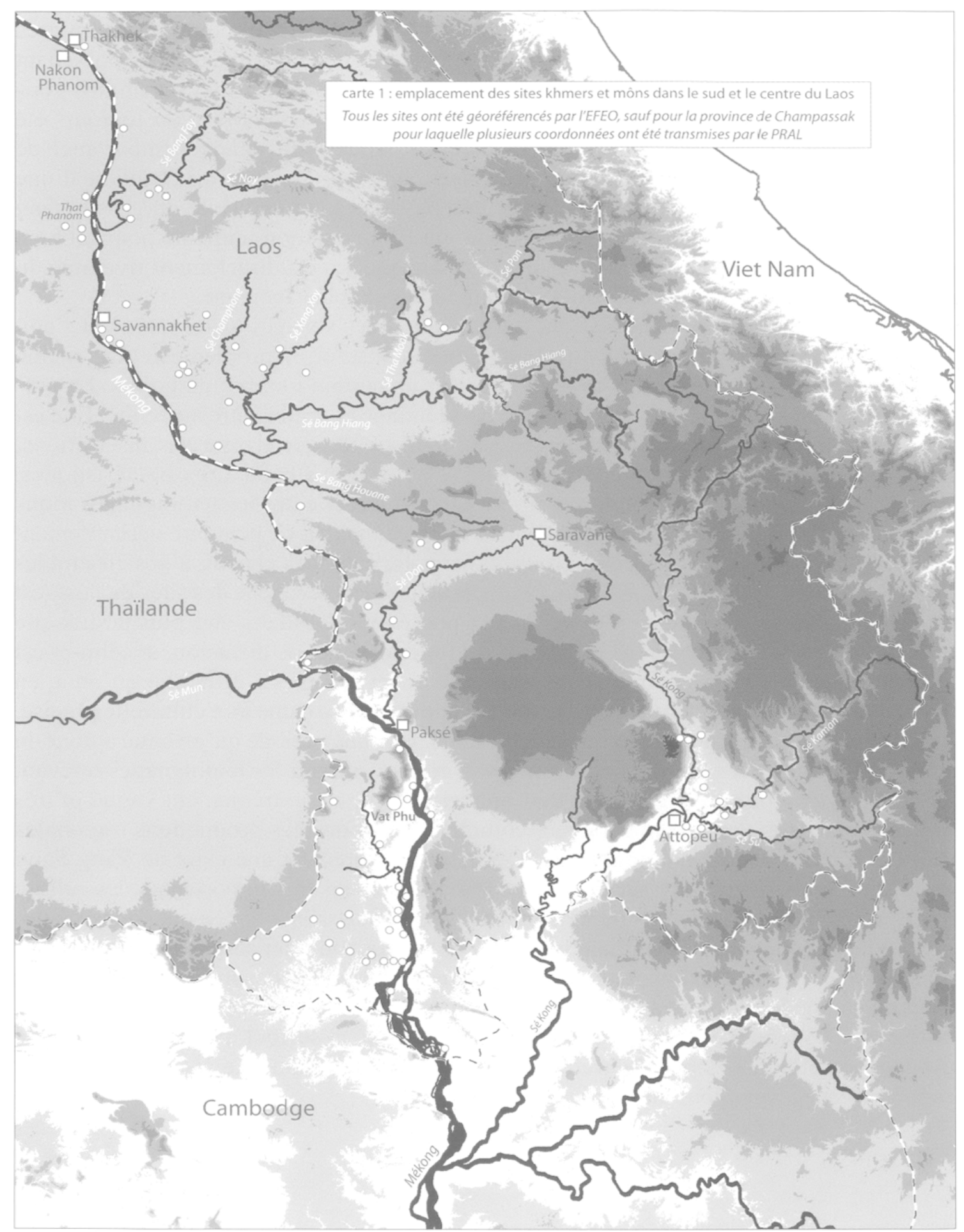

Carte 1 : Emplacement des sites khmers et môns dans le sud et le centre du Laos (J. Ferrand / M. Lorrillard).

Cartes complémentaires à l'article du BEFEO 97-98 («Par-delà Vat Phu. Données nouvelles sur l'expansion des espaces khmer et môn anciens au Laos », p. 205-270) 


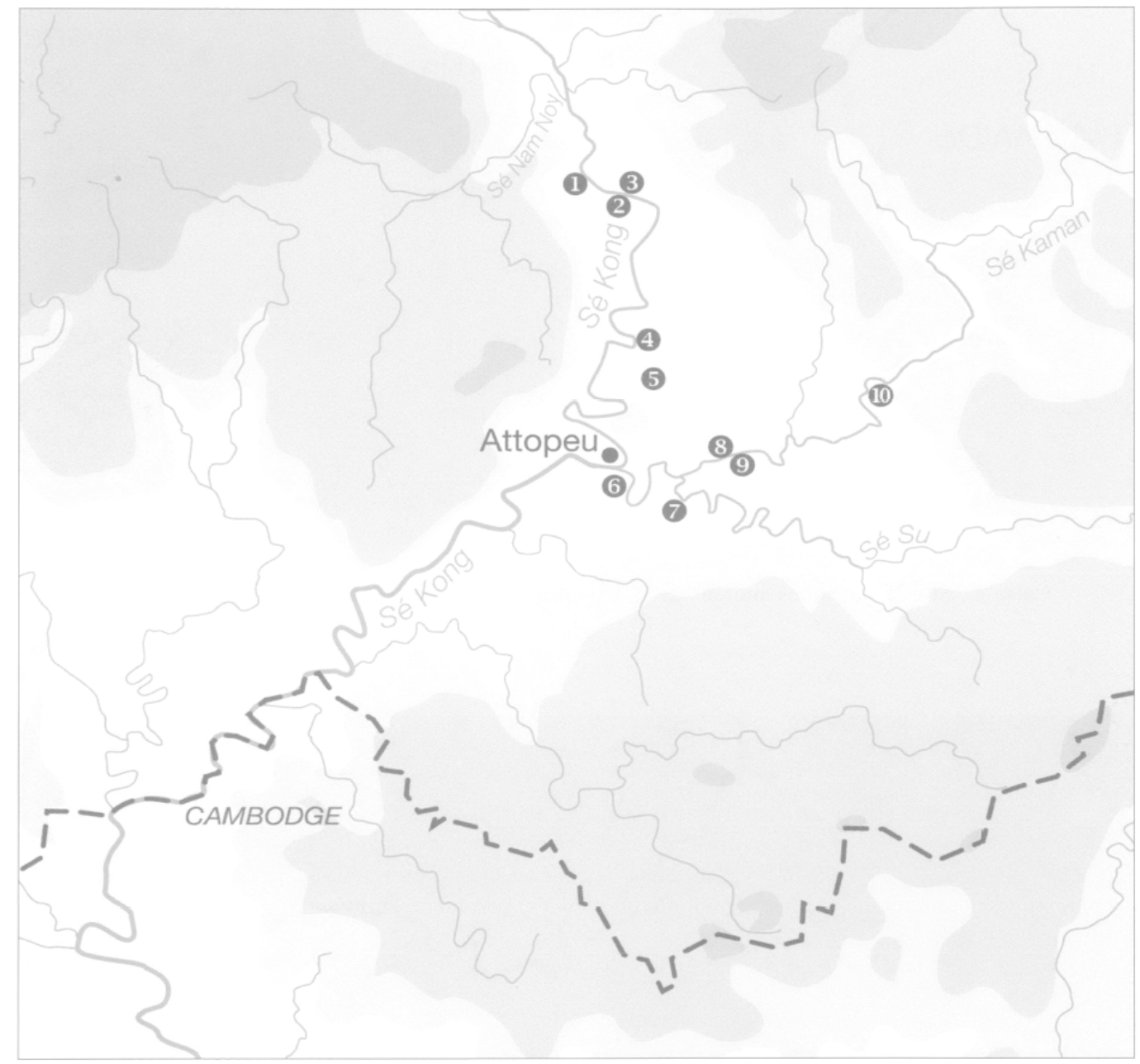

Carte 2 : Sites khmers anciens de la province d'Attopeu (P. Pichard / M. Lorrillard).

1. Ban Khum Kham - 2. Ban Sok - 3. Ban Sapoan - 4. Ban Sakhè - 5. Ban Halang 6. Ban Sé Kaman Nua - 7. Up-mung Sé Su - 8. Ban Fang Daeng - 9. Ban Vat That - 10. Ban Tatkum 


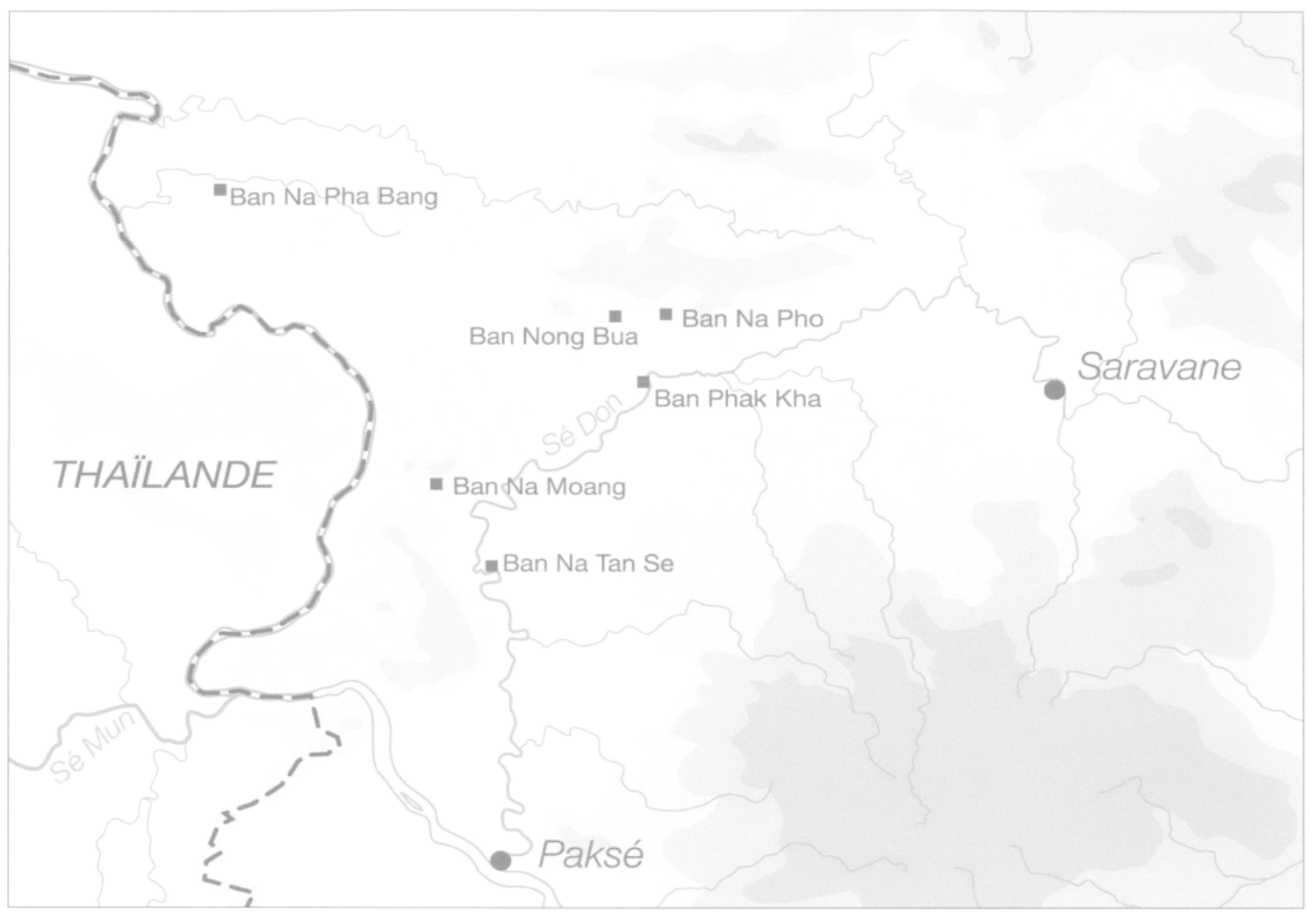

Carte 3 : Sites historiques anciens de la province de Saravane (P. Pichard/M. Lorrillard).

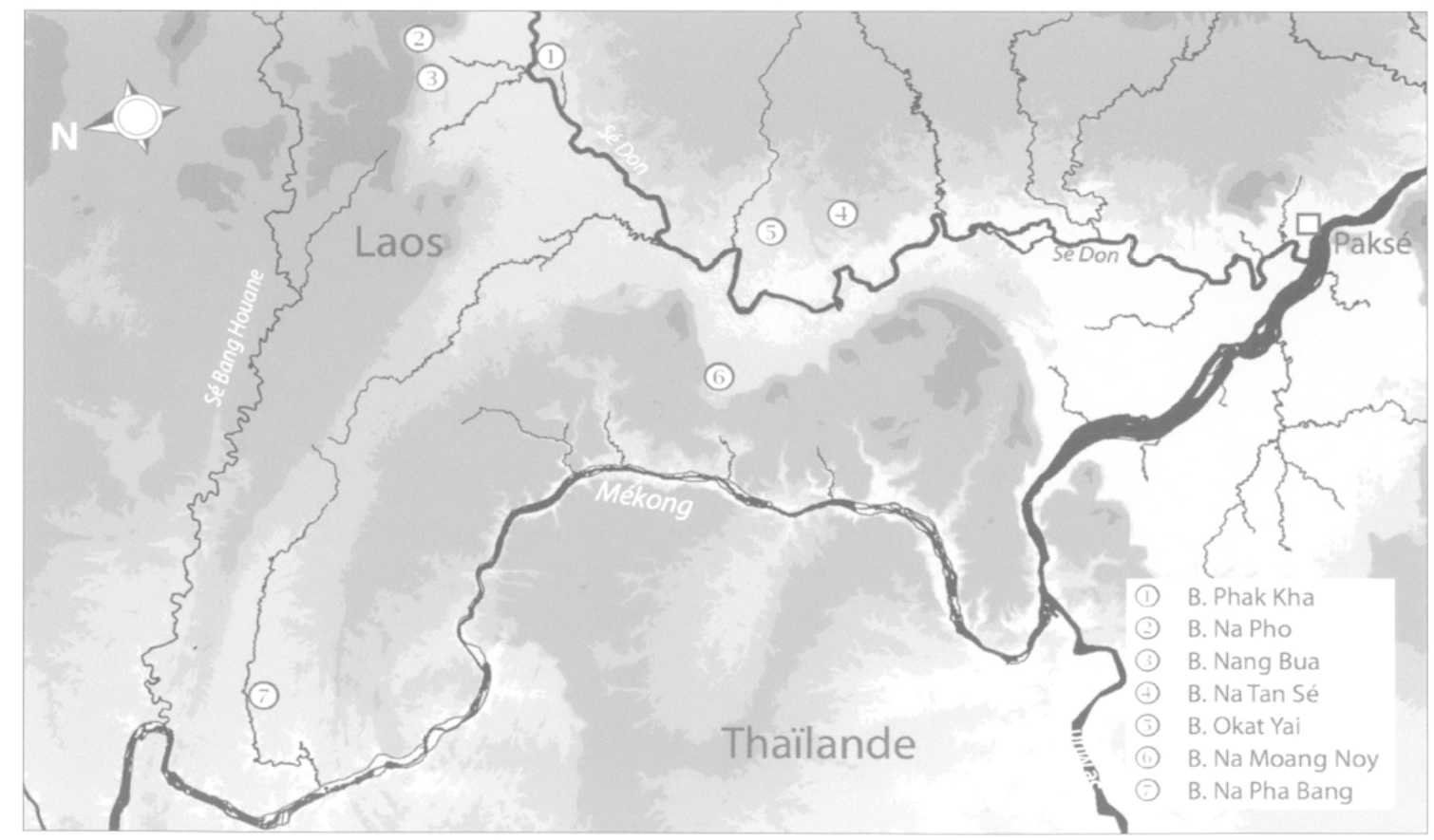

Carte 4 : Corridor de Lakhon Pheng et bassin inférieur de la Sé Don (J. Ferrand/M. Lorrillard). 


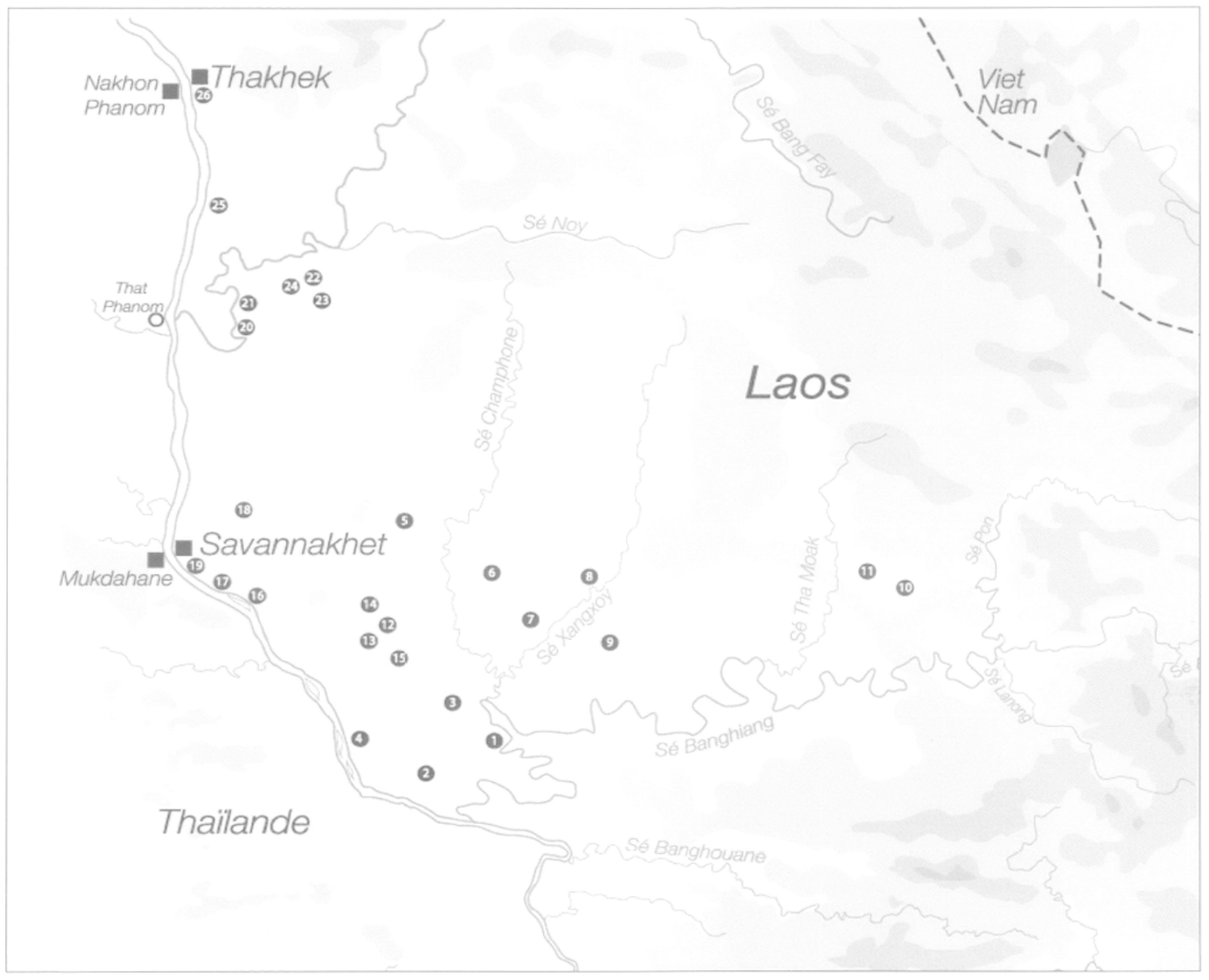

Carte 5 : Sites historiques anciens de la province de Savannakhet (P. Pichard / M. Lorrillard).

1. Ban Hua Hat - 2. Ban Na Khu - 3. Ban Nong Hai - 4. Huan Hin - 5. That $\mathrm{Ku}-6$. Ban Na Kathang -

7. Ban Tum Ye - 8. Ban Non Savang - 9. Ban Muang Phong - 10. Muang Phin - 11. Ban Sé Tamoak12. That Phon-13. Ban Phumma Chedi-14. Ban Don Tum - 15. Ban Tak Daet - 16. Ban Pha Kha Niai 17. Ban Sompoy Noï - 18. That In Hang - 19. Ban Don Seng - 20. Nong Hua Thong - 21. Ban Mak Nao Tai 22. Ban Kang - 23. Ban Na Moang - 24. Ban Sikhai - 25. Ban Xieng Vang -26 . That Sikhot 


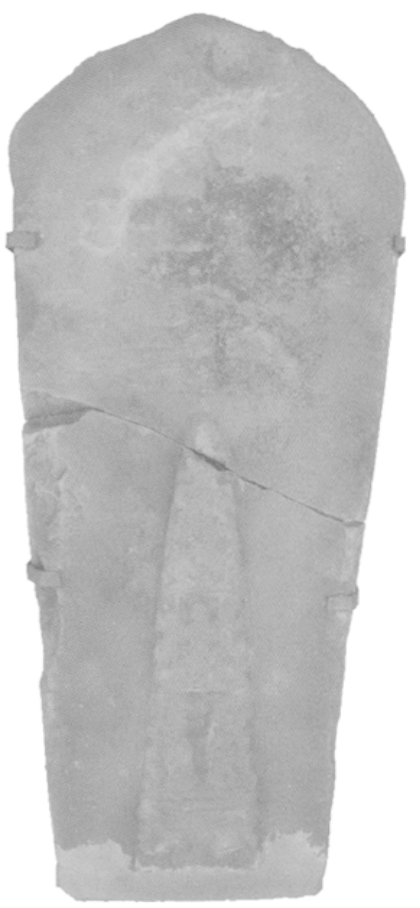

Fig. 1: Sema de Ban Pak Pot. act. au musée du That Luang (cliché M. Lorrillard).

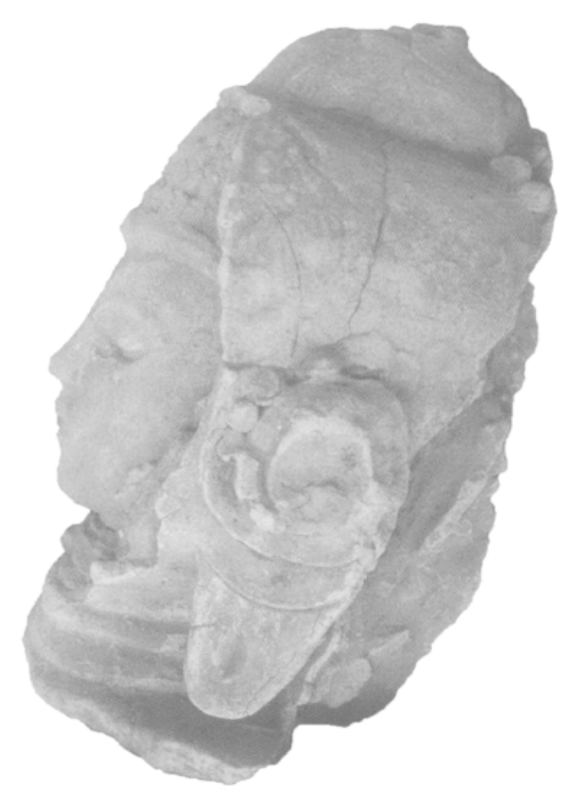

Fig. 3 : Tête de Buddha (ou de divinité) du Vat Thin Kèo. Ban Thin Kèo, act al musée du That Luang (cliche M. Lorrillard).

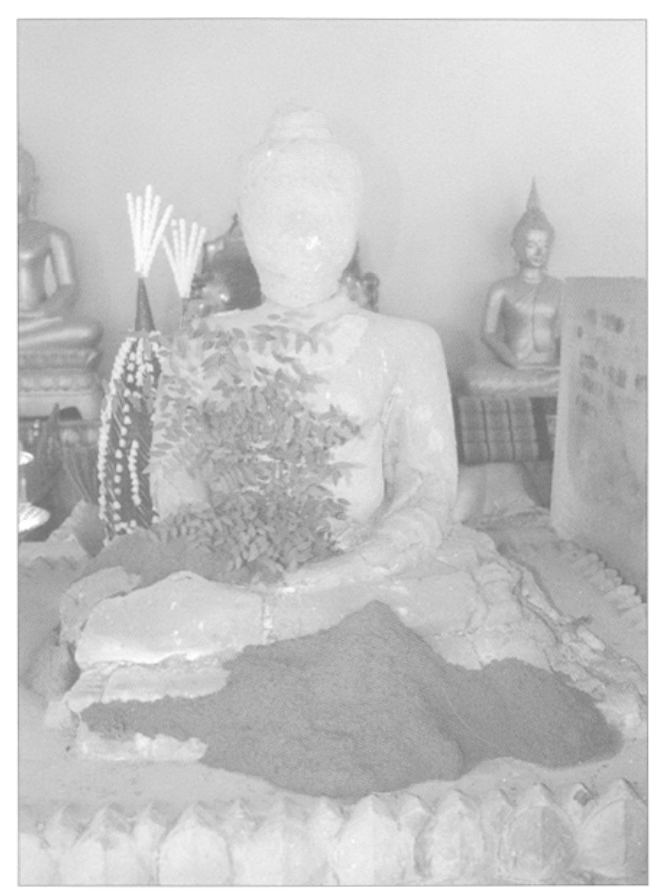

Fig. 2 : Buddha assis du Vat Thin Kèo. Ban Thin Kèo (cliché M. Lorrillard).

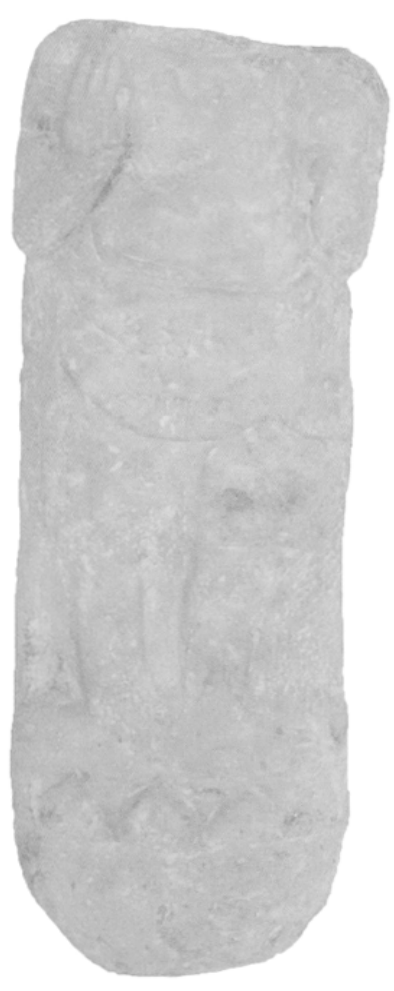

Fig. 4 : Borne avec Buddha du Vat Thin Kèo. Ban Thin Kèo (cliché M. Lorrillard). 


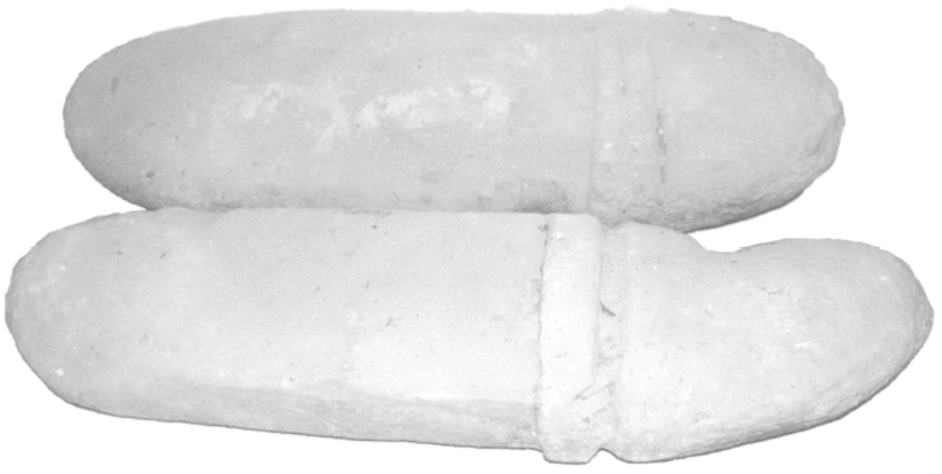

Fig. $5 \Delta$ : Deux sema du Vat Okat, Ban Thin Kèo (cliché M. Lorrillard).

Fig. $6 \triangleright$ : Buddha de B. Thalat. act. au musée du Vat Ho Phra Kèo (cliché M. Lorrillard).

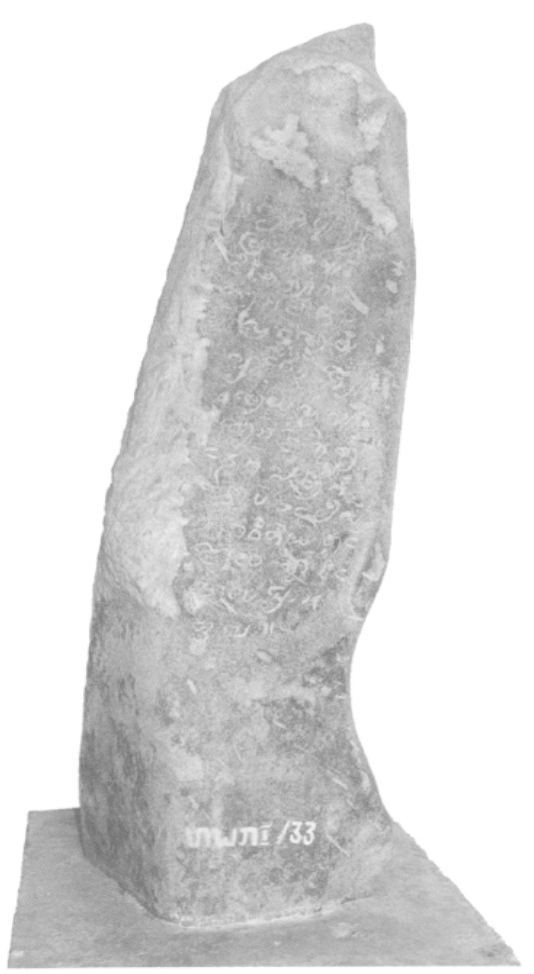

Fig. 7 : Stèle de Ban Thalat, act. au musée du Vat Ho Phra Kèo (cliché M. Lorrillard).
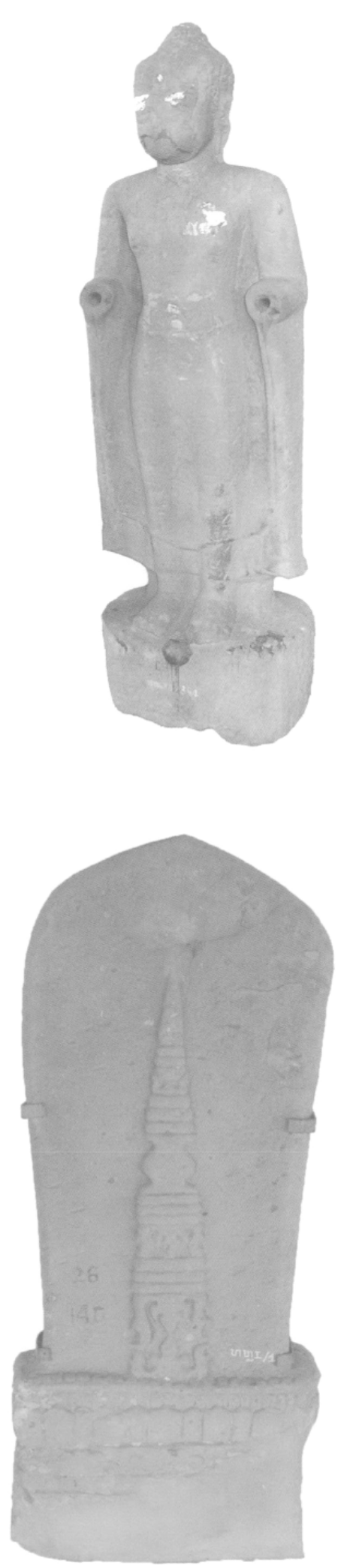

Fig. 8 : Sema du Vat Keng Noy Kao. Ban Muang Kao, act. au musée du That Luang (cliché M. Lorrillard). 


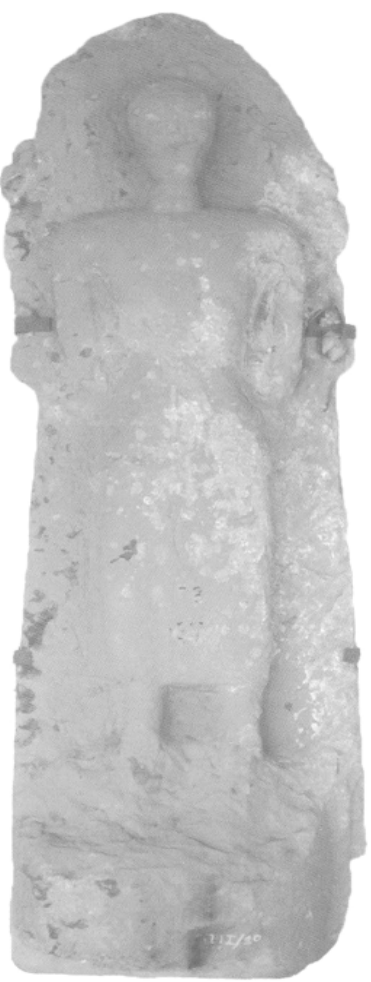

Fig. 9 : Buddha debout adossé à une stèle, Vat Mün Xu, Ban Muang Kao, act. au musée du That Luang. $\mathrm{n}^{\circ}$ TL I/10 (cliché M. Lorrillard).

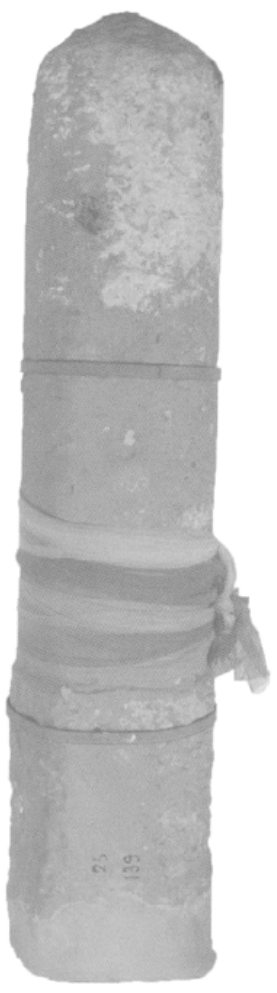

Fig. 10: Sema du Vat Mün Xu, Ban Muang Kao, act. au musće du That Luang, n" TL I/12 (cliché M. Lorrillard).

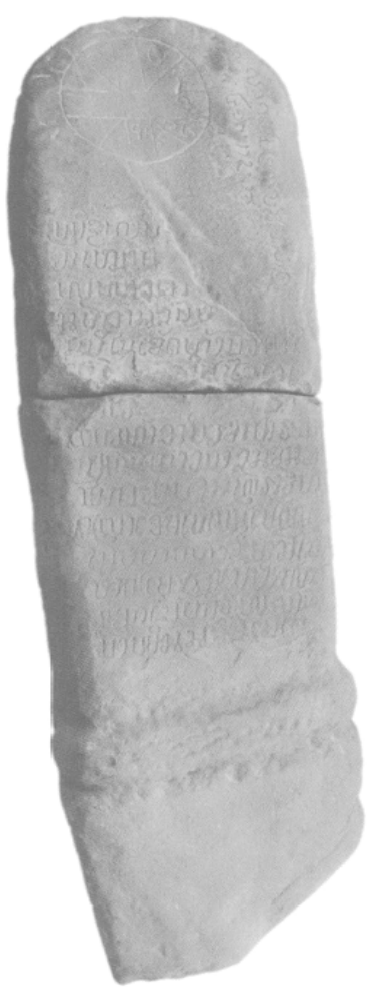

Fig. 11 : Sema réemployé de Ban Vieng Kham, act. au musée du Vat Ho Phra Kèo (cliché M. Lorrillard).

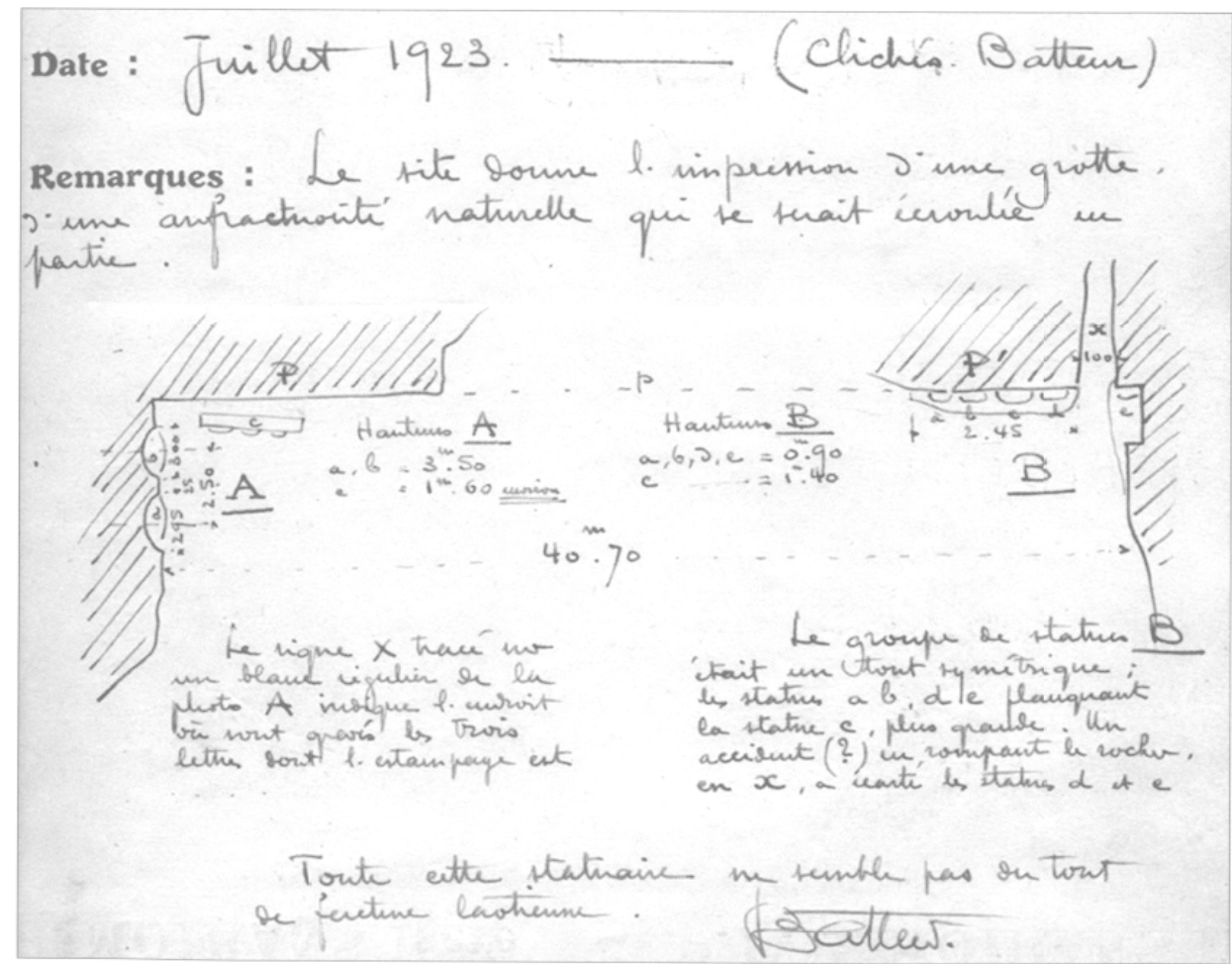

Fig. 12 : Fiche de terrain réalisée par (Ch. Batteur à Vang Sang, en 1923 (cliché EFEO, EFEO_LAO22862a 1). 


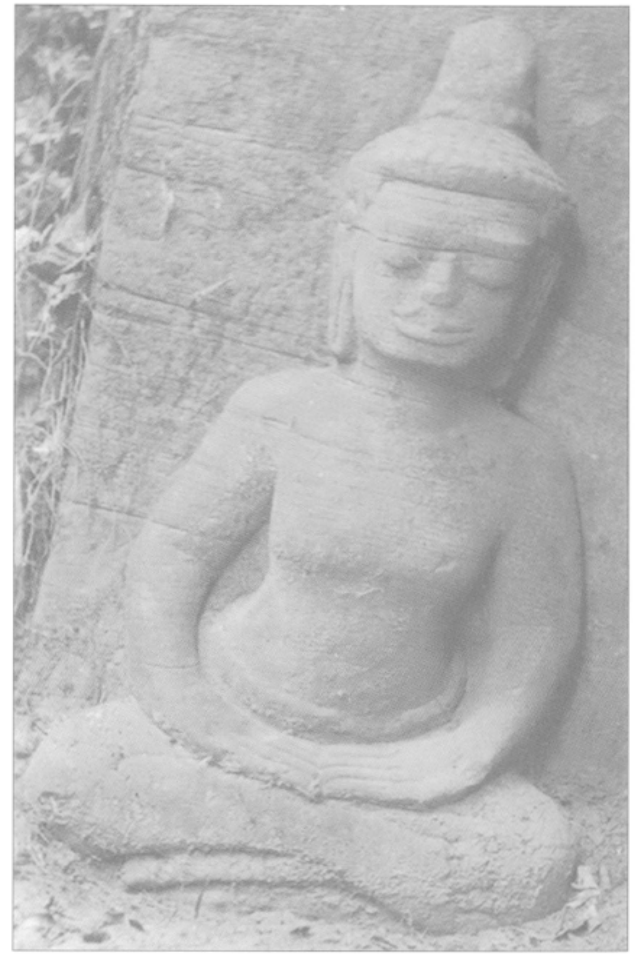

Fig. 13 : Buddha assis adossé à la paroi. Vang Sang, en 1933 (cliché EFEO, EFEO_ LAO20895_1).

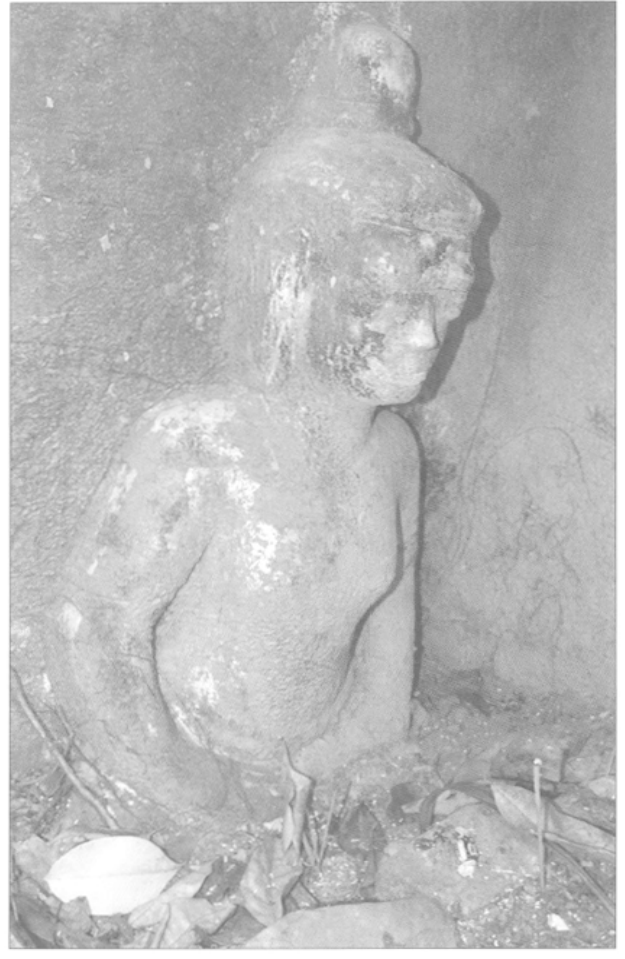

Fig. 14 : Buddha assis adossé à la paroi, Vang Sang, en 2011 (cliché M. Lorrillard).

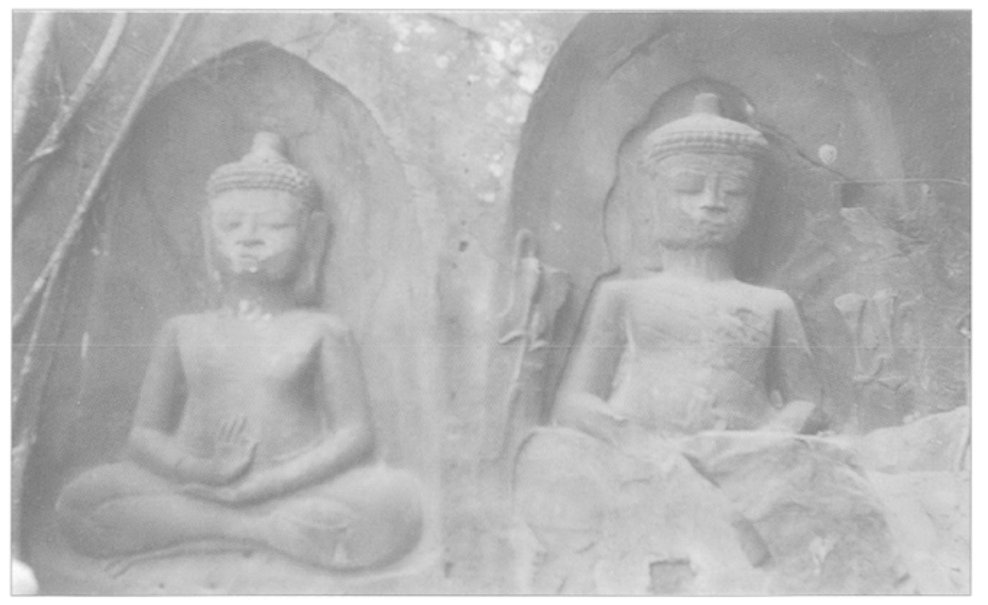

Fig. $15 \Delta$ : Deux grands Buddha adossés à la paroi, Vang Sang. en 1933 (cliché EFEO. EFEO_LAO20895_4).

Fig. $16>$ : Détail d'un motif en bas-relief. Vang Sang, en 1933 (cliché EFEO. EFEO_LAO20895_2).

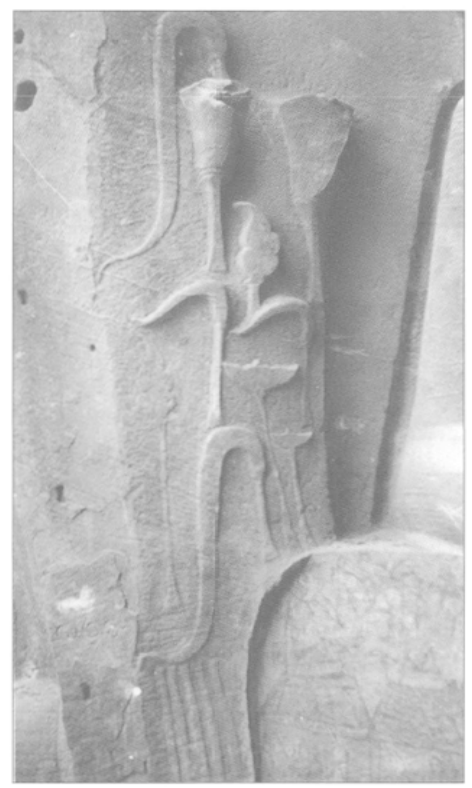




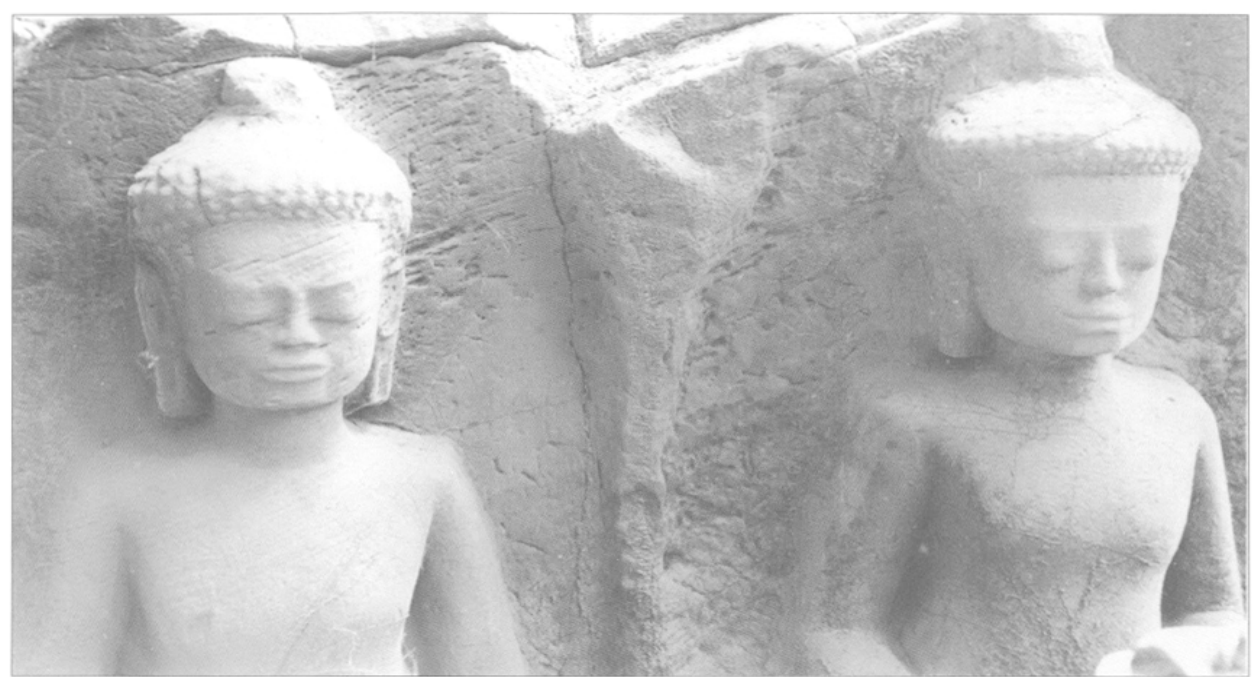

Fig. 17 : Deux Buddha adossés à une roche, Vang Sang, en 1933 (cliché EFEO, EFEO_LAO20937_2).

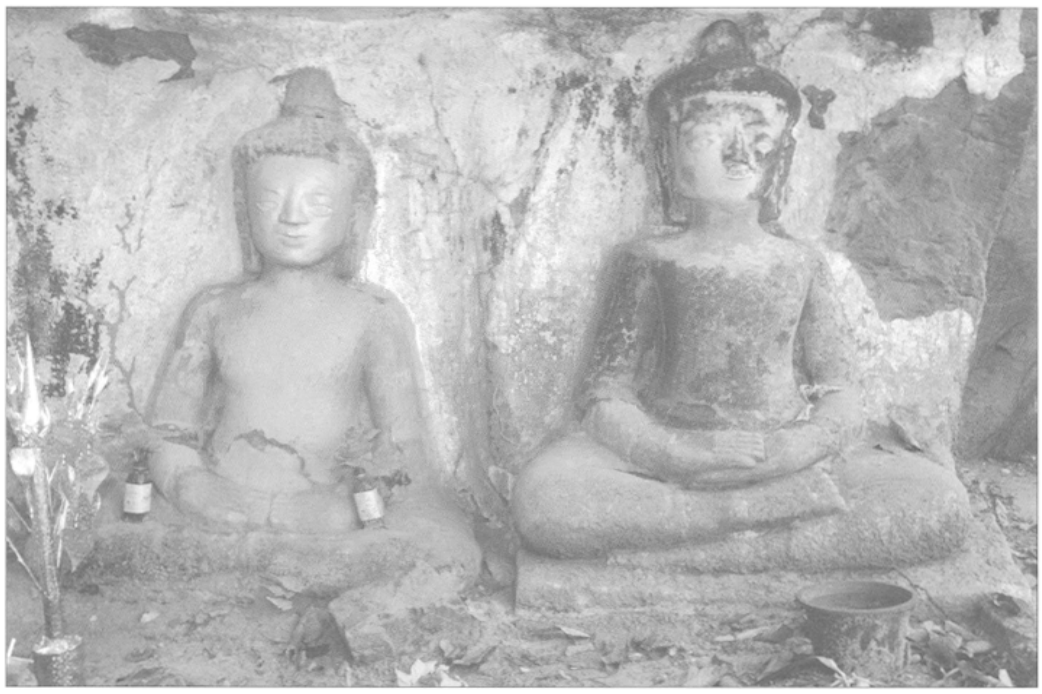

Fig. 18 : Deux Buddha adossés à une roche, Vang Sang, en 2011 (M. Lorrillard).

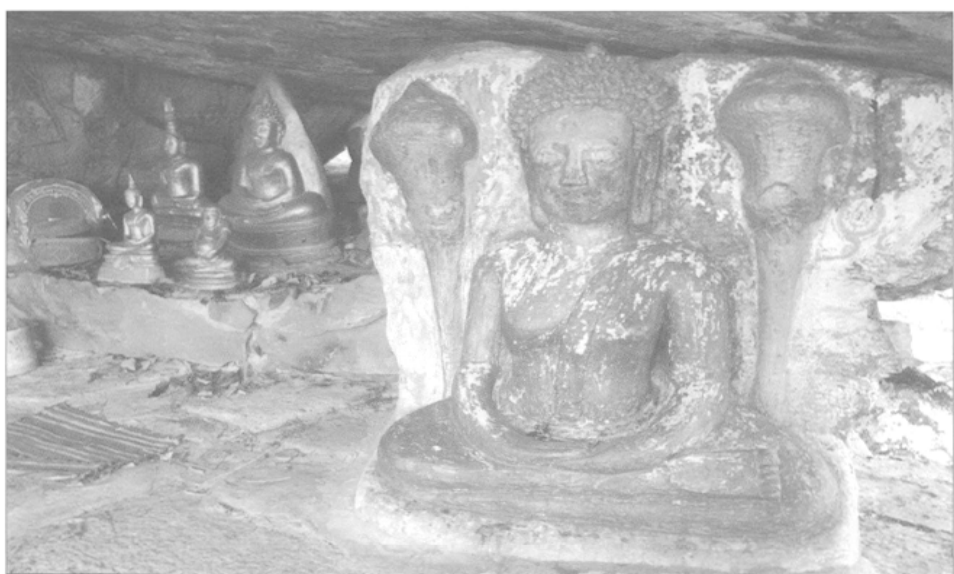

Fig. 19 : Buddha sculpté dans la roche, abri-sous-roche n¹2. Dan Sung (cliché M. Lorrillard). 


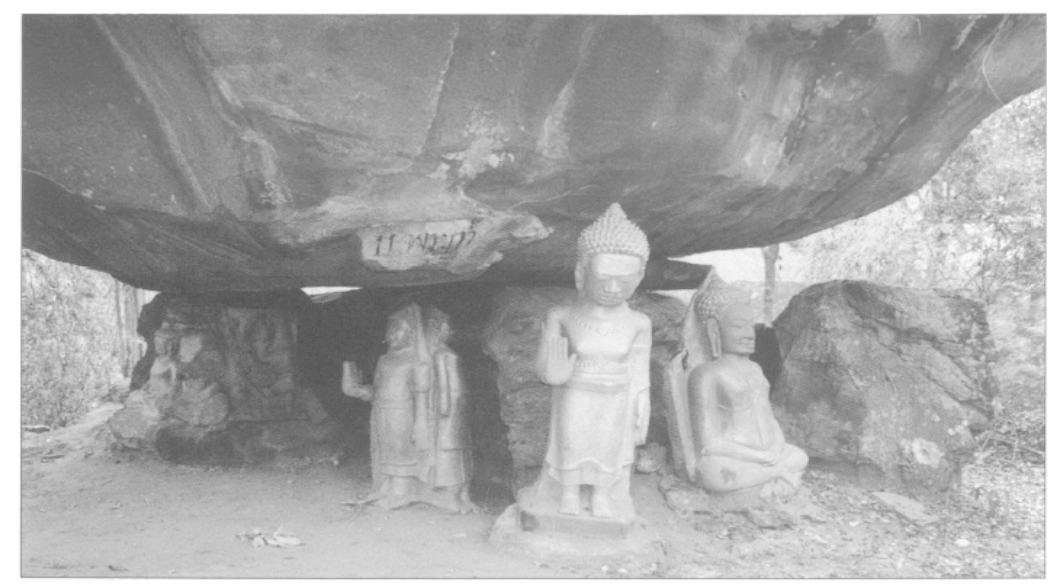

Fig. 20 : Buddha sculptés dans la roche, abri-sous-roche n 11, Dan Sung (cliché M. Lorrillard).

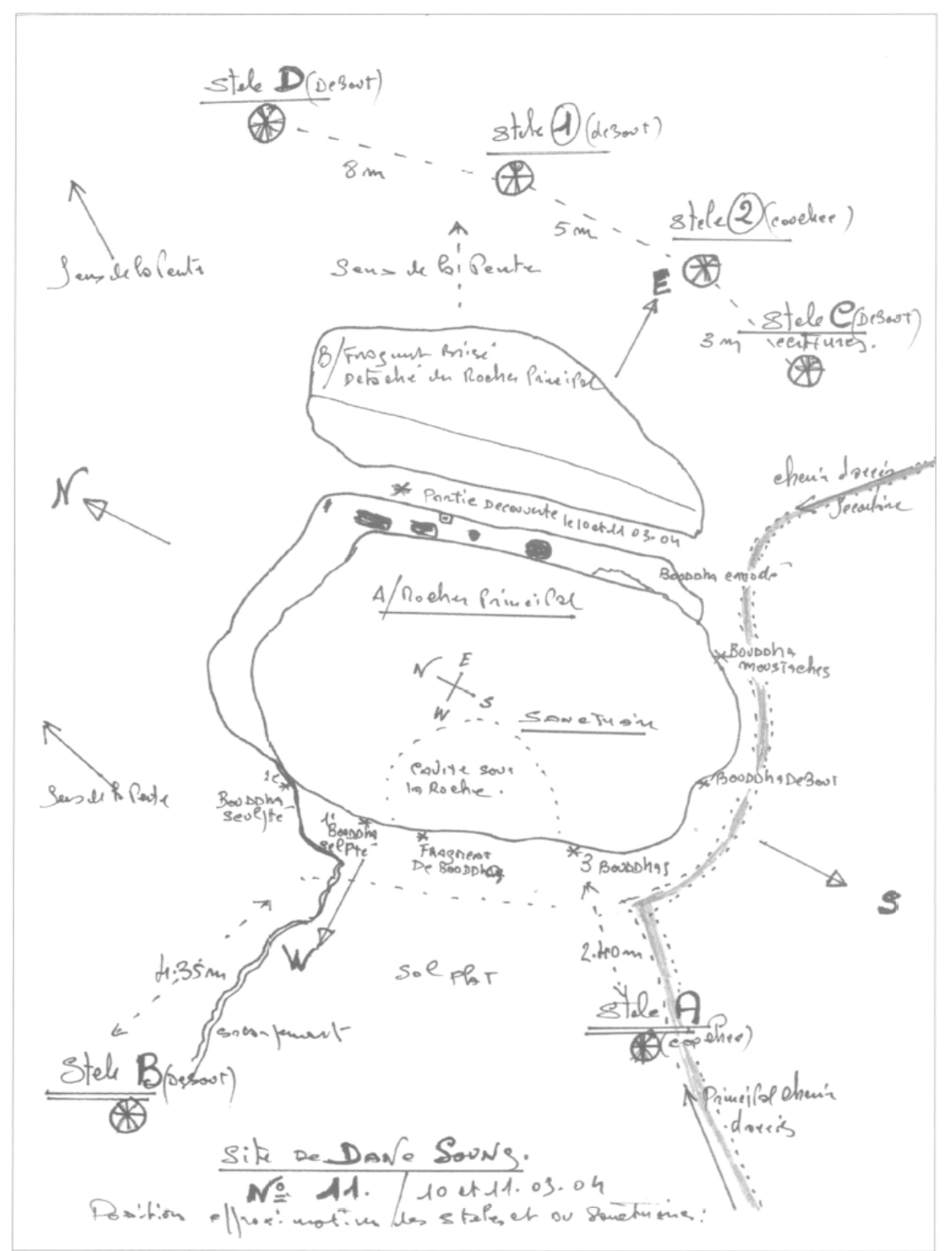

Fig. 21 : Plan schématique de l'abri-sous-roche n¹1, Dan Sung (schéma B. Brunetti, EFEO). 


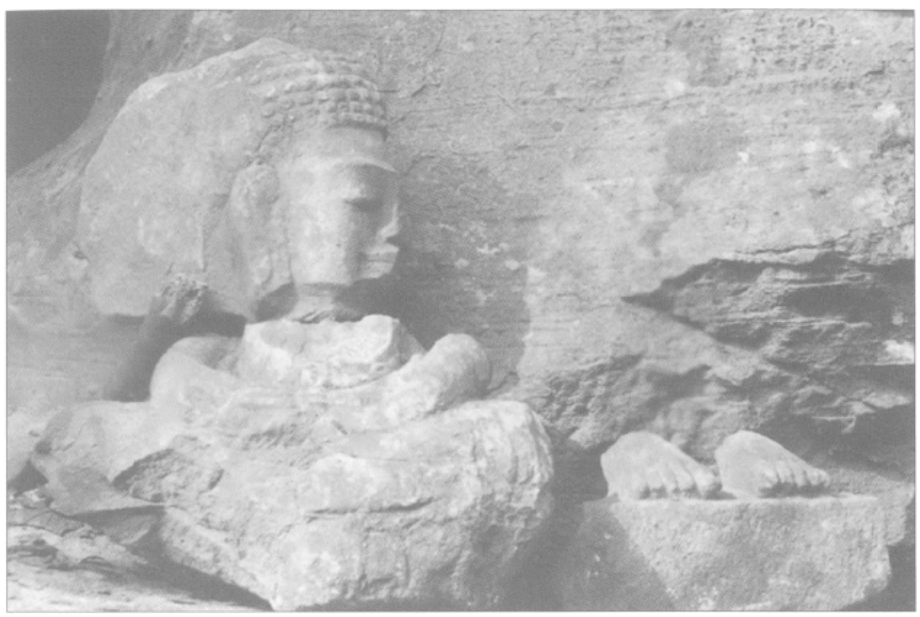

Fig. 22 : Fragments de Buddha, abri-sous-roche n¹1, Dan Sung, en 1933 (cliché EFEO, EFEO_LAO20948_4).

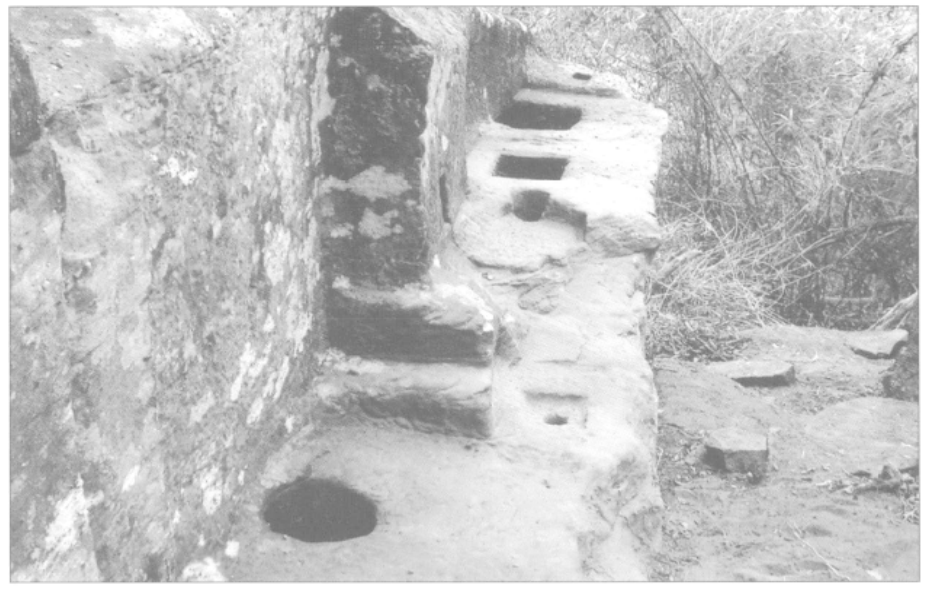

Fig. 23 : Côté nord de l'abri-sous-roche n"11, Dan Sung (cliché M. Lorrillard).

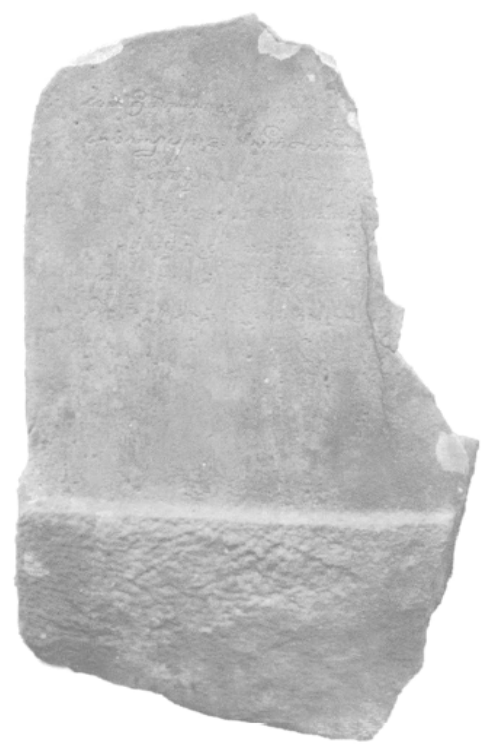

Fig. 24 : Sema inserit, Dan Sung (cliché M. Lorrillard). 


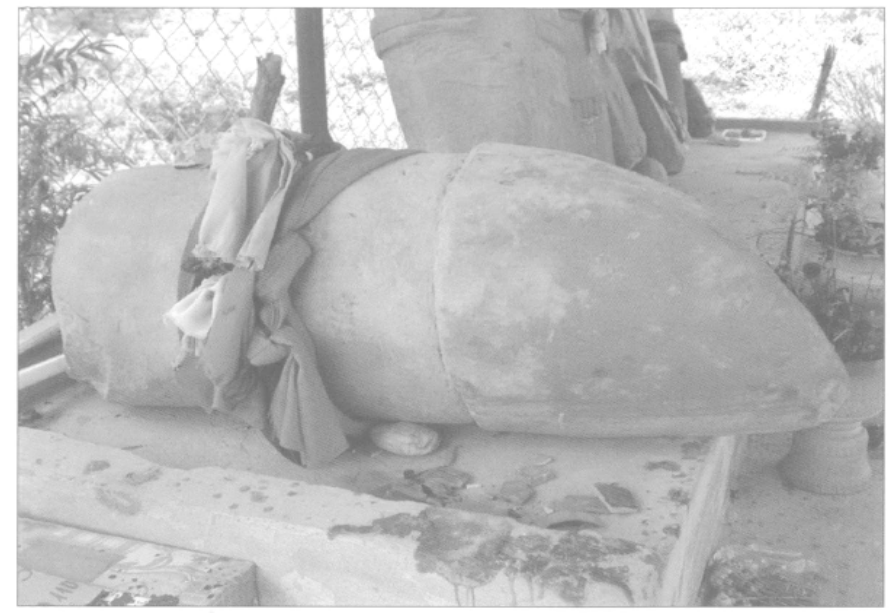

Fig. 25 : Sema, Ban Na Sone (cliché M. Lorrillard).

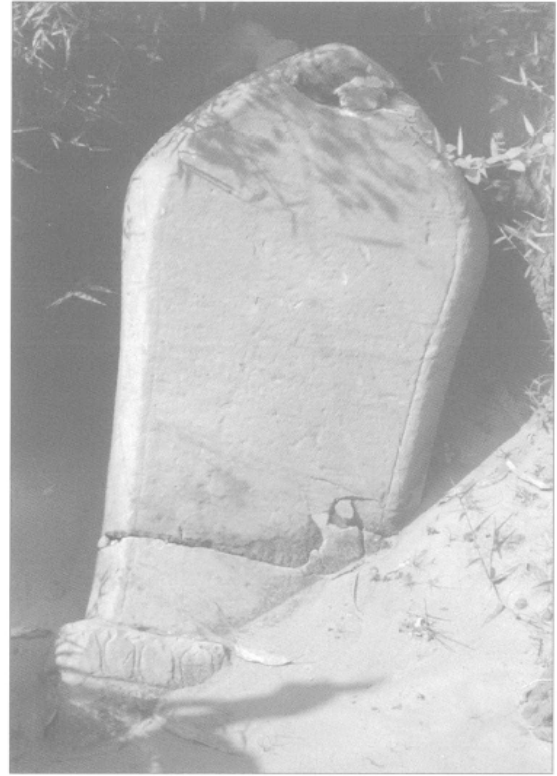

Fig. 26 : Sema, Ban Na Sone (cliché M. Lorrillard).

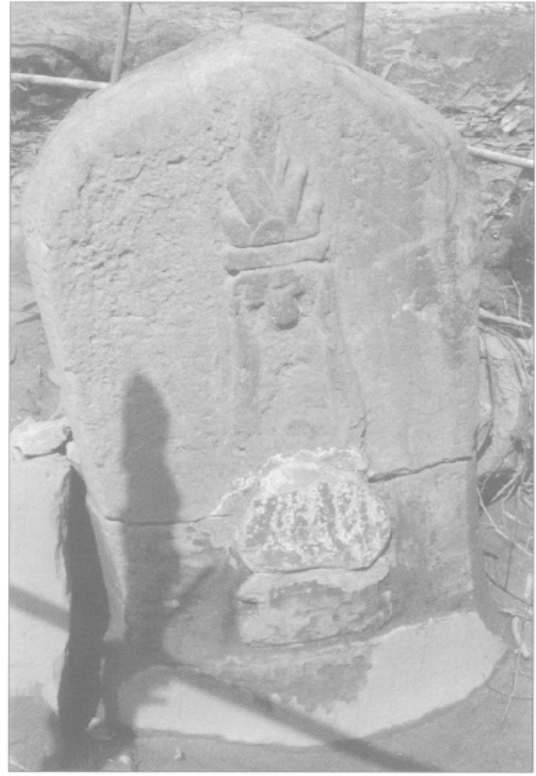

Fig. 27 : Sema, Ban Na Sone (cliché M. Lorrillard).

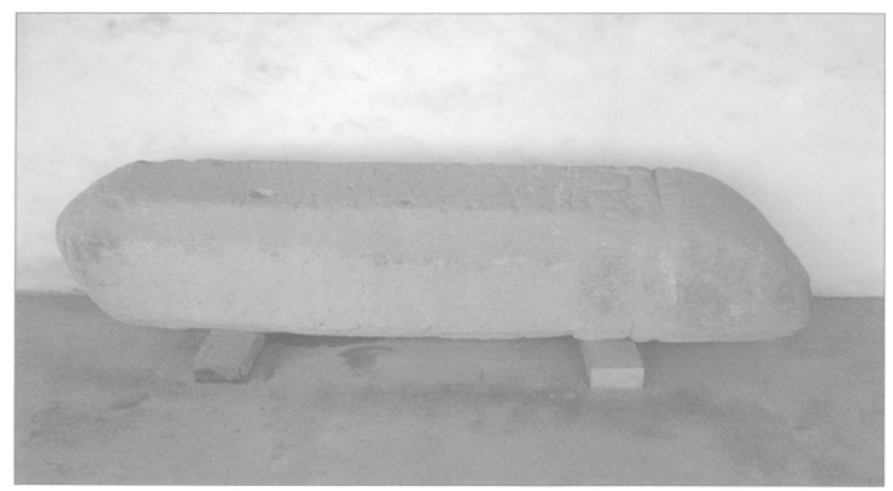

Fig. 28 : Sema, Ban Sapheu. act. au musée du That Luang (cliché M. Lorrillard). 


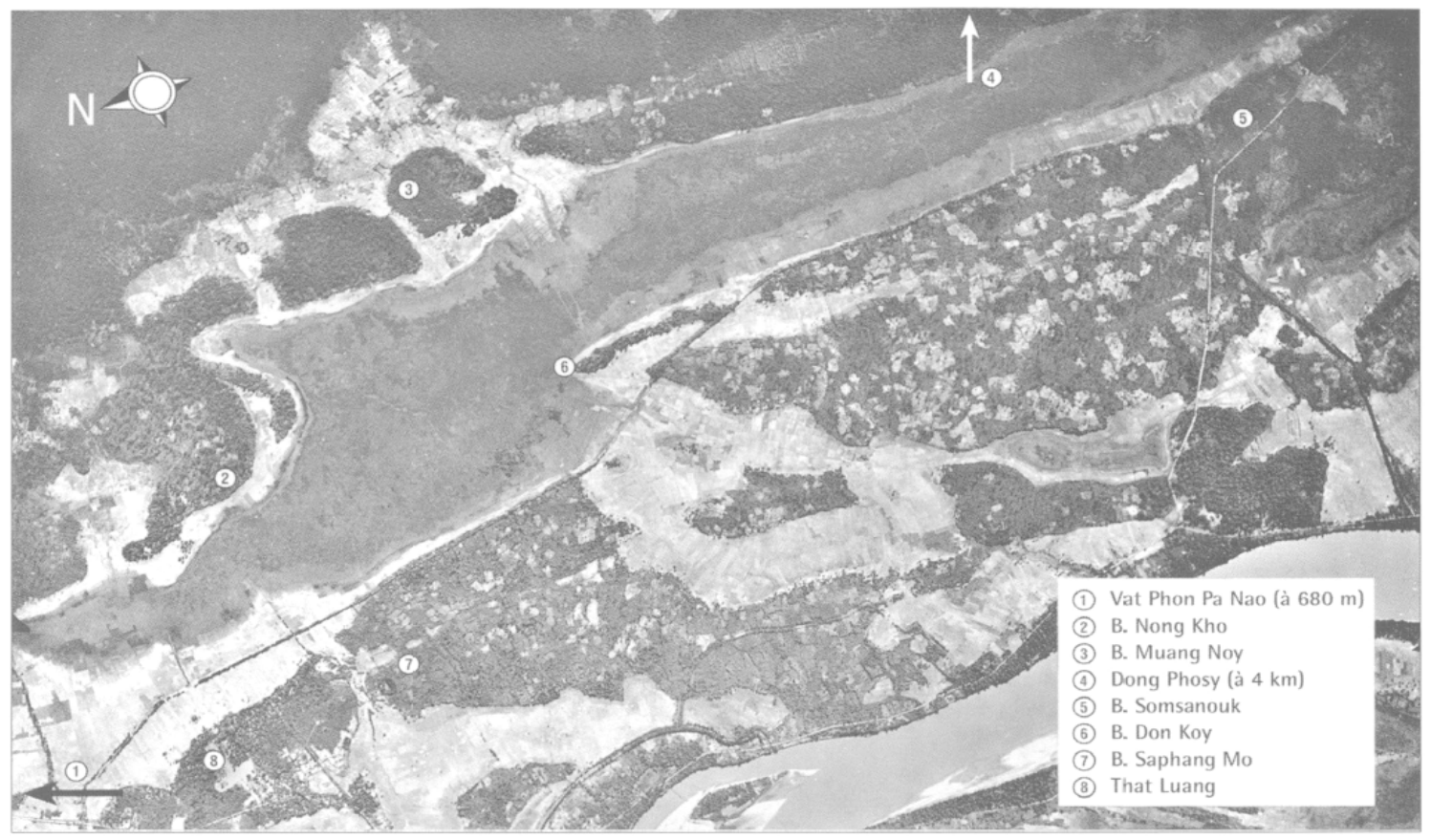

Fig. 29 : Les sites môns des bords du marais du That Luang (cliché du fonds Williams-Hunt, 1946).

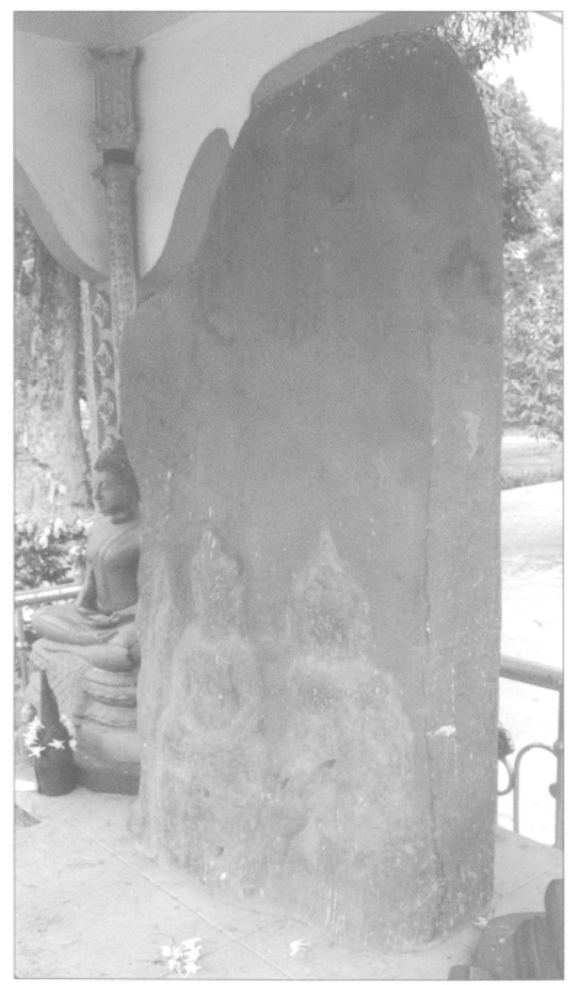

Fig. 30 : Sema historié, Vat Phon Pa Nao, Vientiane (cliché M. Lorrillard).

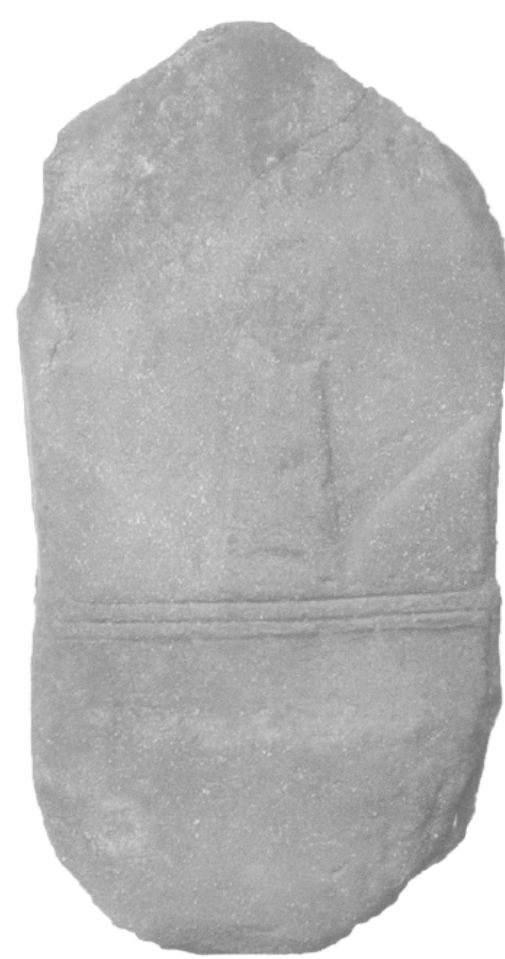

Fig. 31 : Sema, Ban Non Kho (cliché M. Lorrillard). 


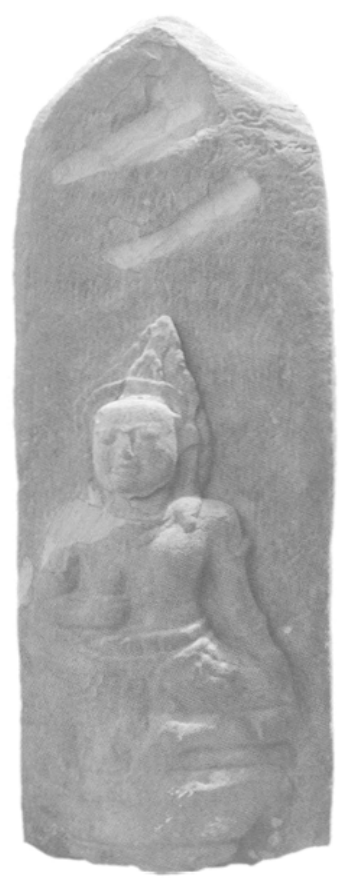

Fig. 32 : Fragment supćrieur d'un sema historié et inscrit, Ban Dong Phosy, act. au musée du Vat Ho Phra Kèo (cliché M. Lorrillard).

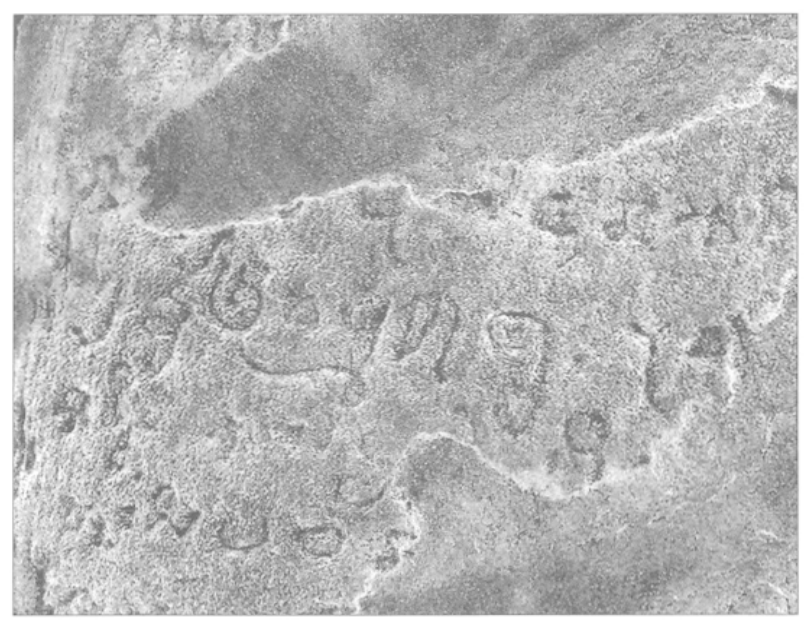

Fig. 33 : Détail de l'inscription du sema historié de Ban Dong Phosy (cliché M. Lorrillard).

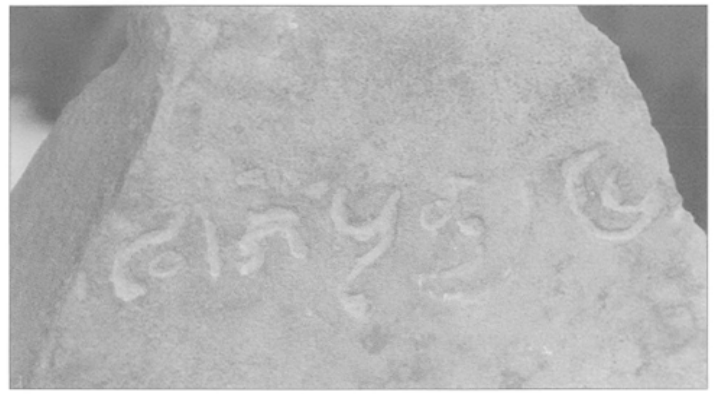

Fig. 34 : Sema inscrit de Ban Dong Phosy, act. au musée du Vat Ho Phra Kèo (cliché M. Lorrillard). 


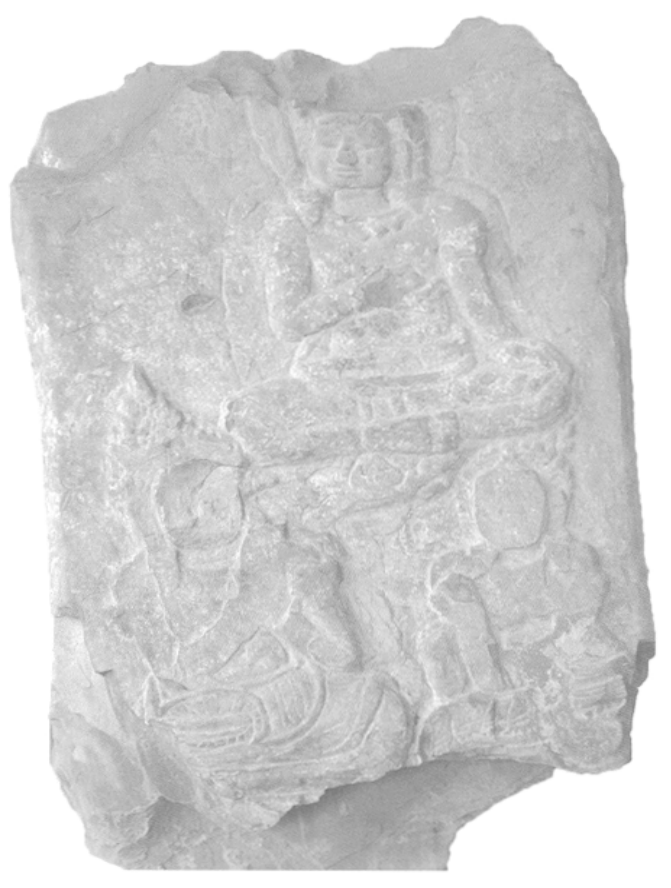

Fig. 35 : Sema historié n" 1 de Ban Saphang Mo (cliché M. Lorrillard).

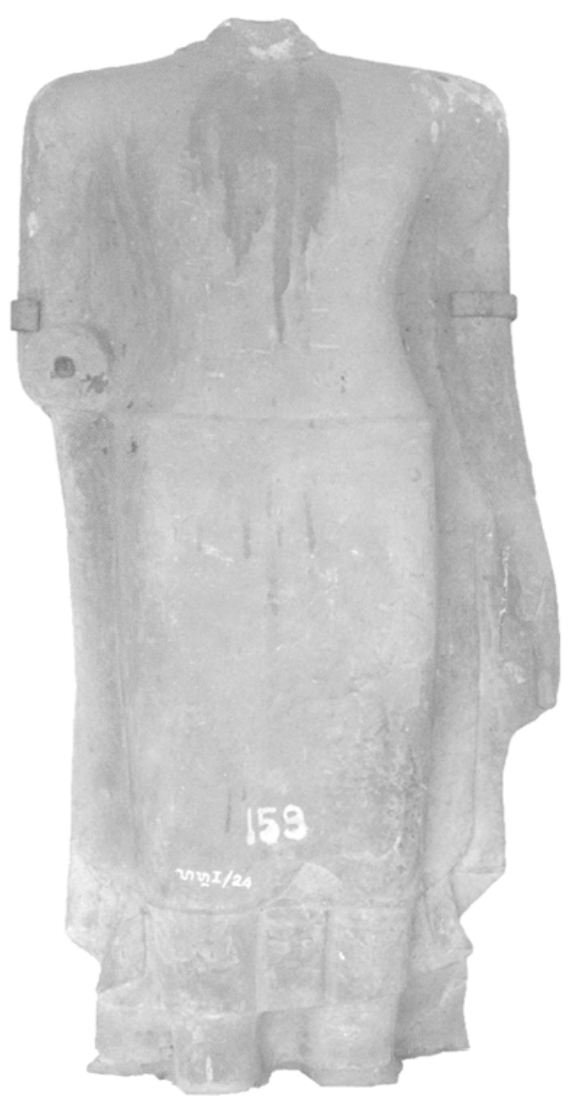

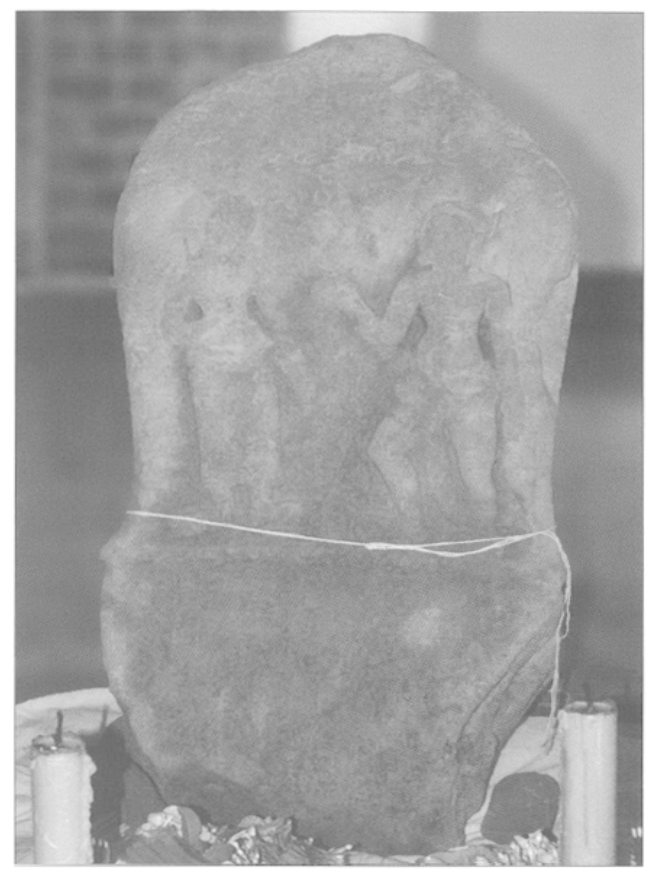

Fig. 36 : Sema historié n“2 de Ban Saphang Mo (cliché M. Lorrillard).

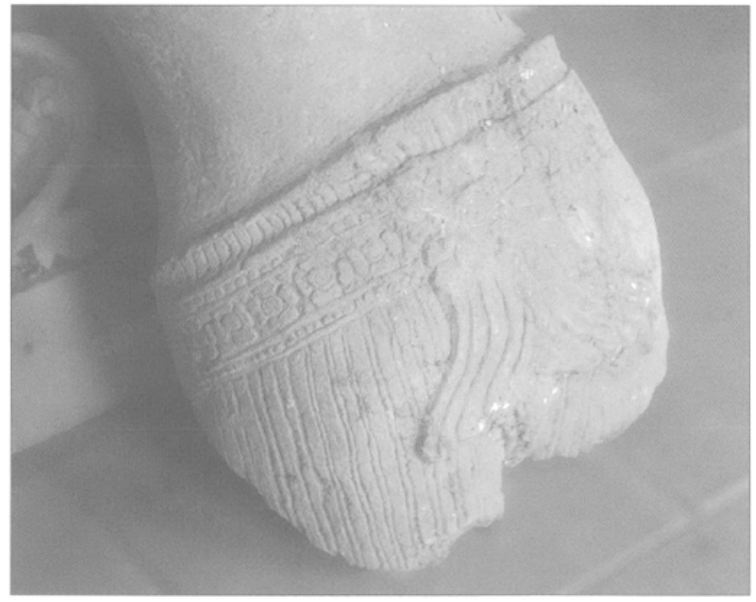

Fig. $38 \Delta$ : Détail du côté postérieur d'un personnage masculin en ronde-bosse, musée du That Luang. n" TL 1/38 (cliché M. Lorrillard).

Fig. 37 : Buddha debout, musée du That Luang. n' TL 1/24 (cliché M. Lorrillard). 


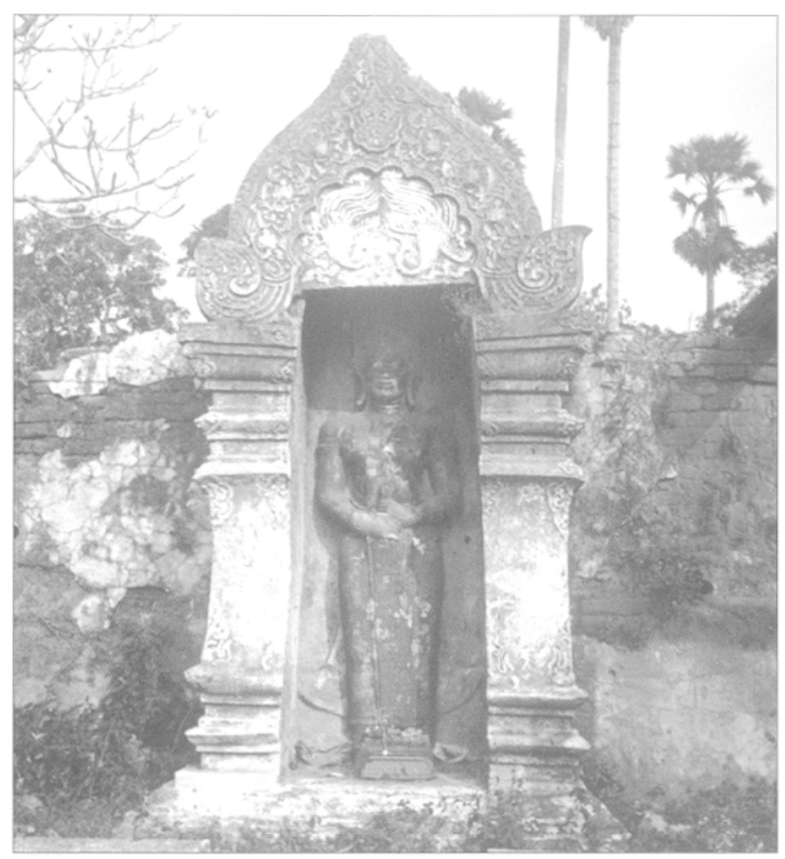

Fig. 39 : Niche avec dvārapäla. That Luang (cliché EFEO, fonds Parmentier, EFEO_PARH03774).

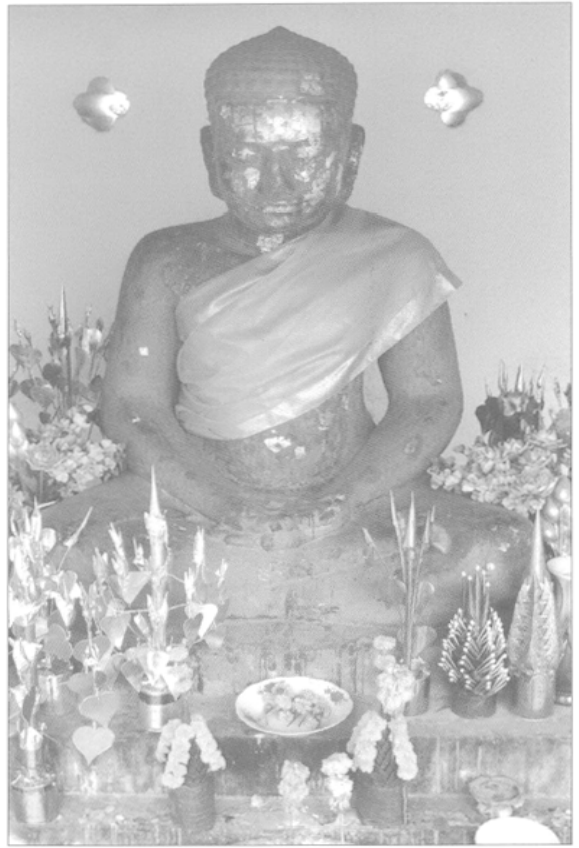

Fig. 40 : Statue présumée de Jayavarman VII, musée du That Luang (cliché M. Lorrillard).

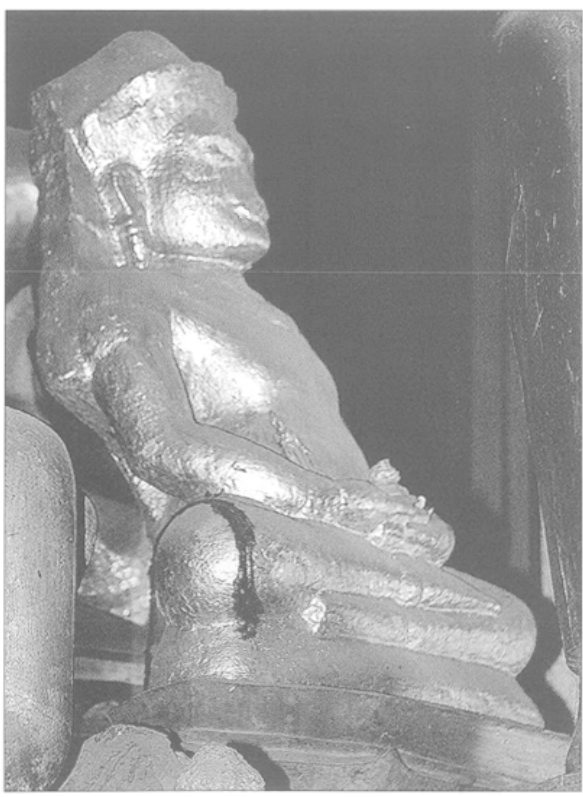

Fig. 41 : Buddha paré, Vat Si Muang. Vientiane (cliché M. Lorrillard).

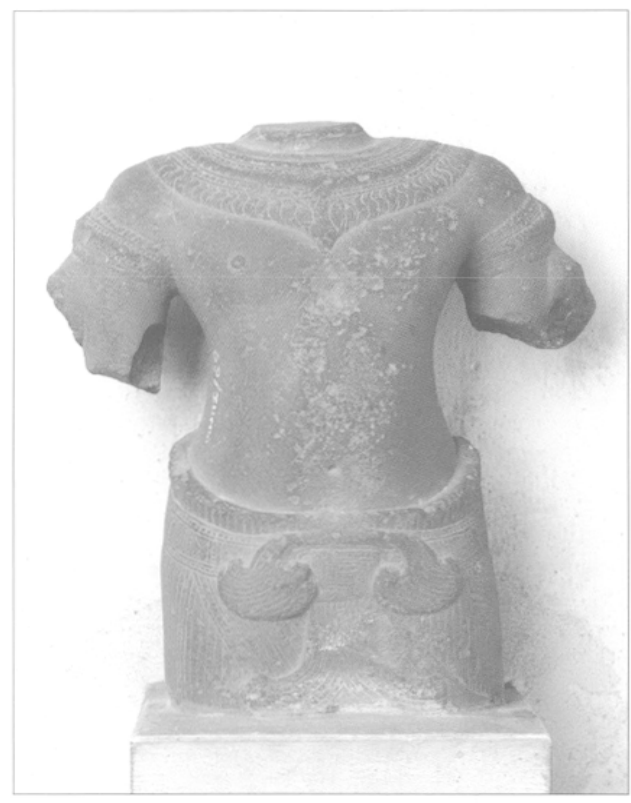

Fig. 42 : Personnage masculin, musée du Vat Ho Phra Kèo, n" HPK I/69 (cliché EFEO Vientiane). 

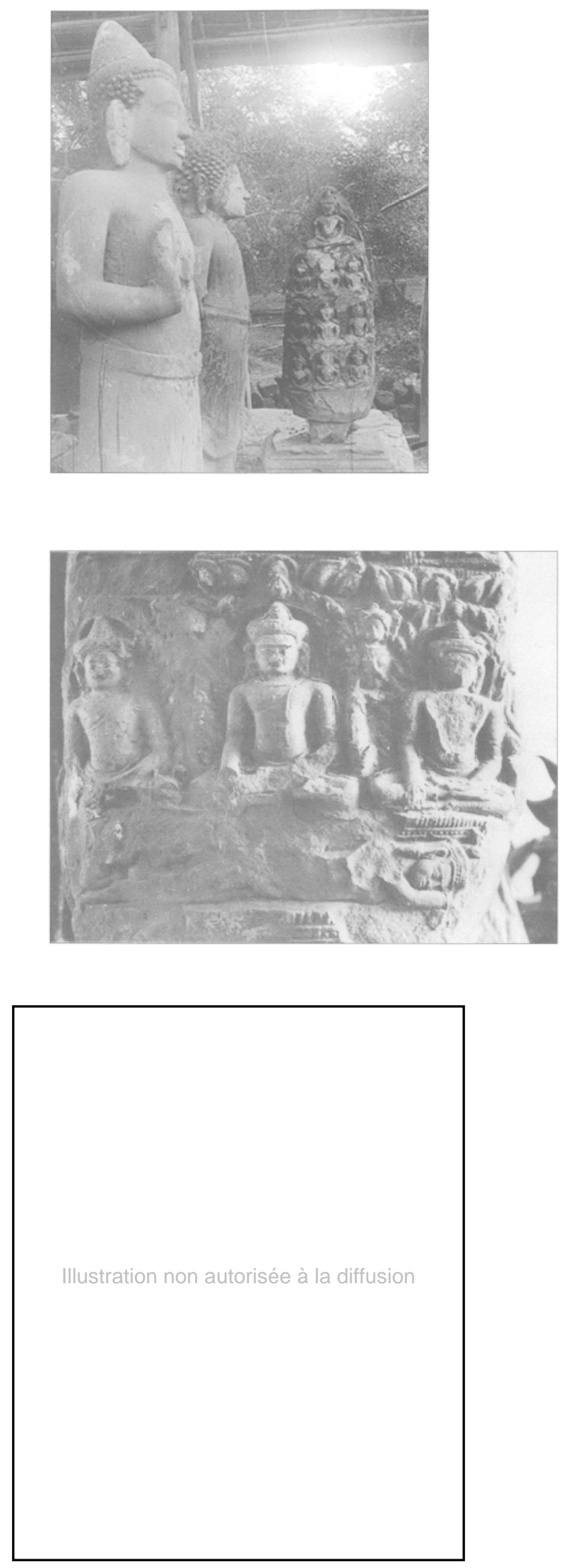

Fig. 43 : Deux Buddha et borne bouddhique du Vat Inpeng, Vientiane (cliché EFEO, fonds Parmentier, EFEO PARH00597).

Fig. 44 : Détail d'une borne bouddhique. Vat Inpeng. Vientiane (cliché EFEO. EFEO_LAO22454_1).

Fig. 45 : Sculptures bouddhiques, Vat Inpeng. Vientiane (cliché H. W. Woodward Jr., 1965). 
Fig. 46 : Stèle et Buddha de Say Fong (cliché EFEO, EFEO_LAO21902).

Fig. 47 : Personnage (Mañjuśrī ?) adossé à une stèle, musée du Vat Ho Phra Kèo, n"HPK I/71 (cliché EFEO Vientiane).

Fig. 48 : Personnage (Mañjuśrī ?) adossé à une stèle, musée du Vat Ho Phra Kèo, $n^{\circ}$ HPK I/65 (cliché EFEO Vientiane).
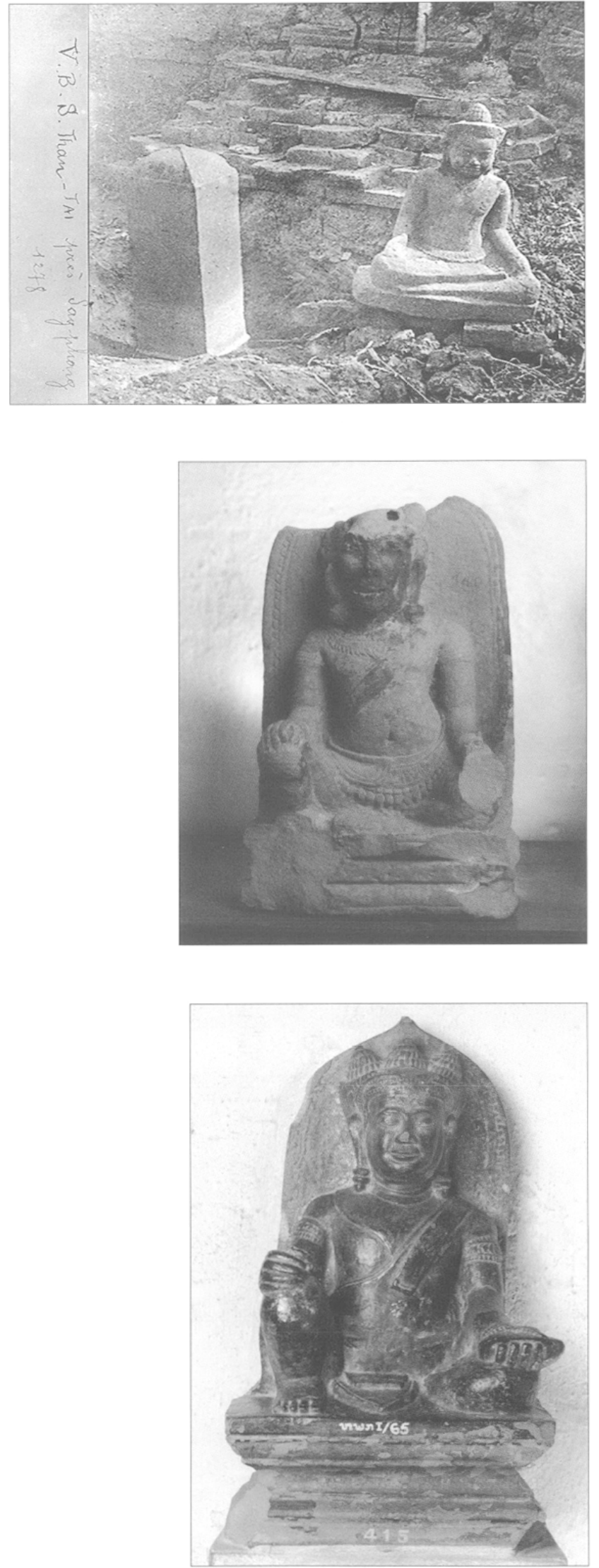


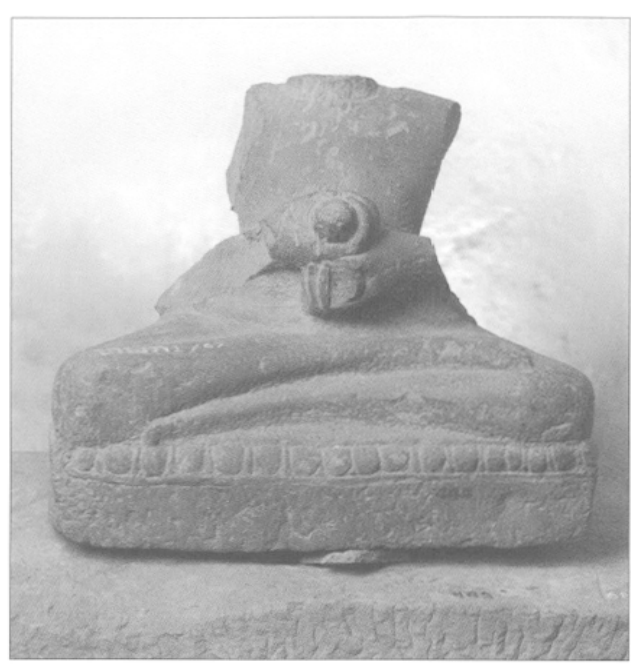

Fig. 49 : Vajrapāṇi, musée du Vat Ho Phra Kèo, n HPK I/67 (cliché EFEO Vientiane).

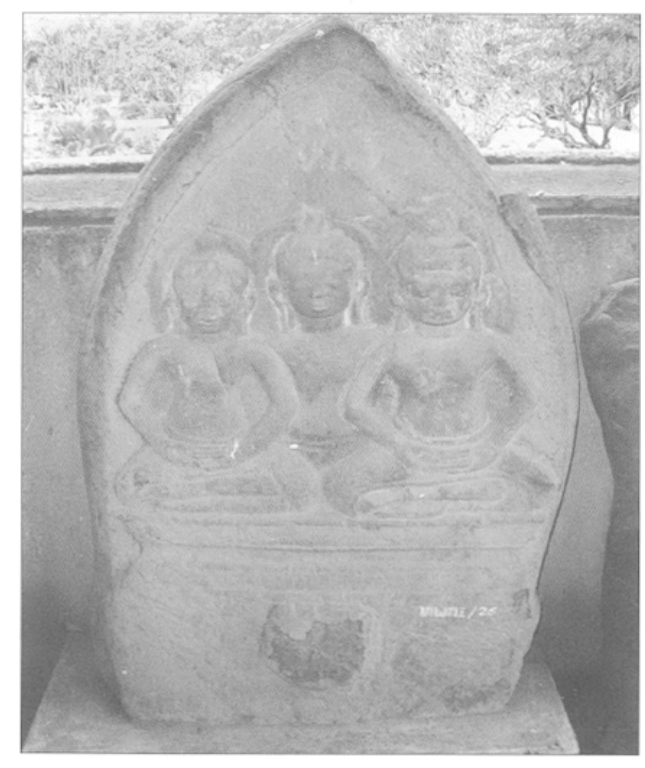

Fig. 50 : Stèle avec trois Buddha, musée du Vat Ho Phra Kèo, nHPK I/26 (cliché M. Lorrillard).

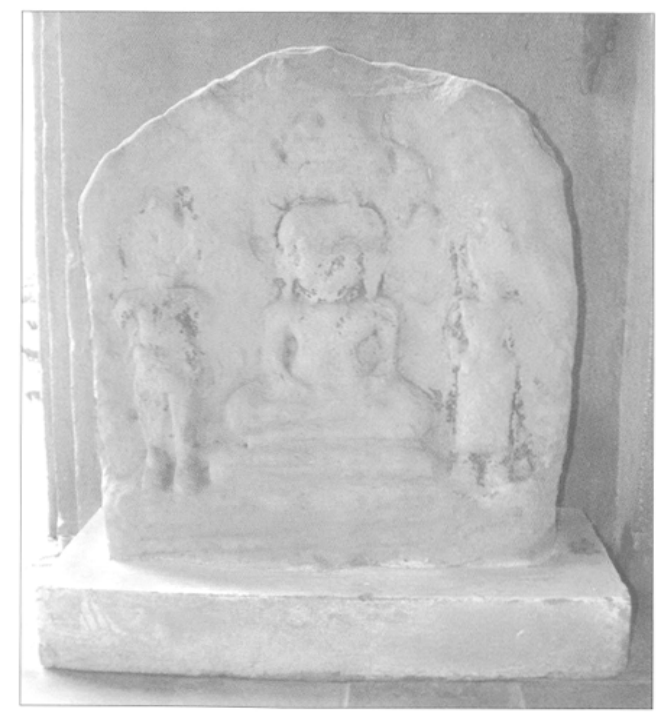

Fig. 51 : Stèle avec triade bouddhique, musée du Vat Ho Phra Kèo (cliché M. Lorrillard). 
Fig. 52 : Sema de Ban Pak Thon (cliché M. Lorrillard).

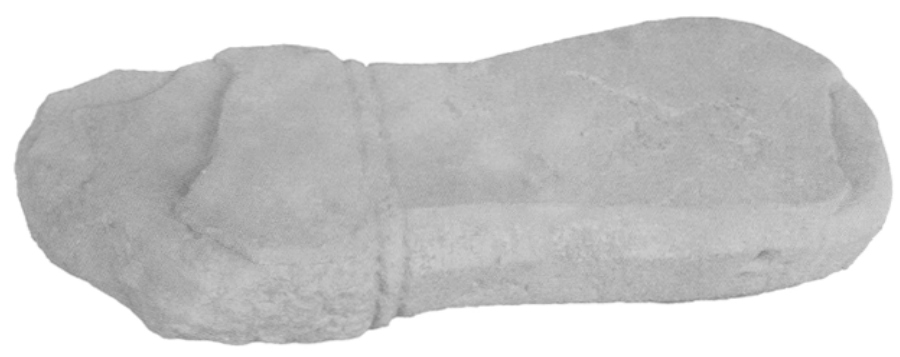

Fig. 53 : Sema du Vat Si Phum. Sanakham (cliché M. Lorrillard).

Fig. 54 : Deux Buddha assis sur nāga du Vat Niai Pha Hot, Sanakham (cliché M. Lorrillard).
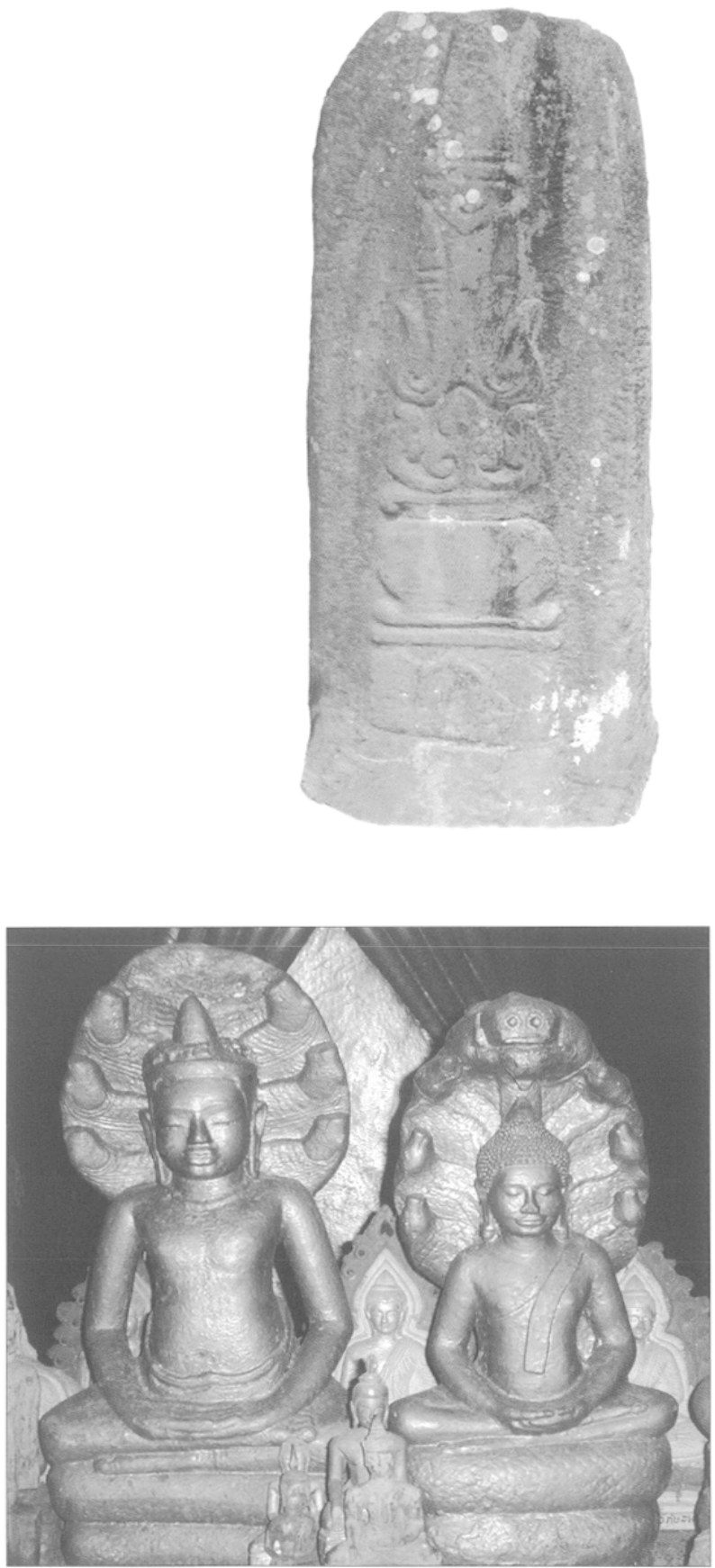